\title{
Unexpected Ortho-Heck Reaction under the Catellani Conditions
}

\author{
Alexander J. Rago and Guangbin Dong* \\ Department of Chemistry, University of Chicago, Chicago, Illinois 60637, United States \\ *Email: gbdong@uchicago.edu
}

\section{Table of Contents}

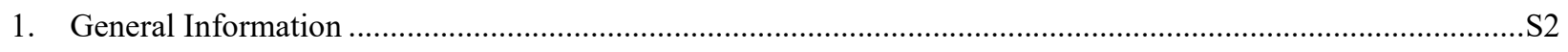

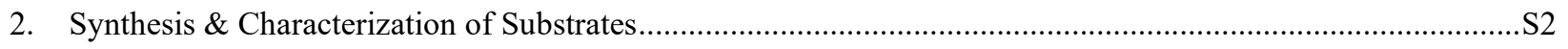

3. Experimental Procedures for the Ortho-Heck Reactions.................................................................................. 4

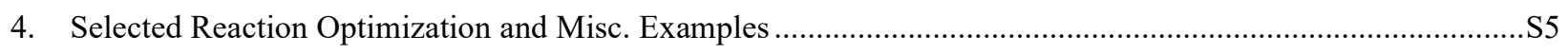

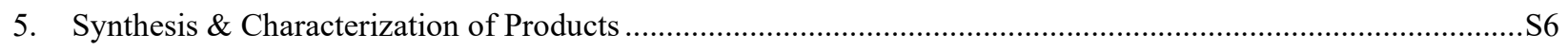

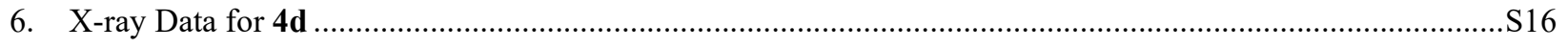

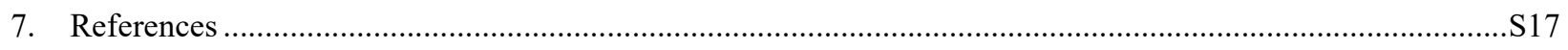

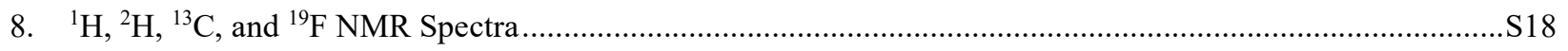




\section{General Information}

All reaction vials were flame-dried and allowed to cool to room temperature while capped in order to remove as much moisture as possible from the glass surface. $\mathrm{Pd}(\mathrm{dba})_{2}$ was purchased from Chem Impex Intl. Inc. and Sigma-Aldrich. Extra-dry dimethylformamide (DMF) used in the key reactions was purchased from Acros Organics. All commercially available substrates were used without further purification. All reactions were carried out in vials (test-scale reactions, $4 \mathrm{~mL}$ vials; isolation-scale reactions, $8 \mathrm{~mL}$ vials; $1.0 \mathrm{mmol}$-scale reaction, $20 \mathrm{~mL}$ vial) unless otherwise noted. All reactions that required heating were conducted on hot plates using appropriately sized heating blocks for vials and the temperature was monitored via a thermometer submerged in a vial filled with silicone oil (instead of the hot plate's temperature probe reading). Thin layer chromatography (TLC) analysis was run on silica gel plates purchased from EMD Chemical (silica gel 60, F254). Infrared spectra were recorded on a Nicolet iS5 FT-IR Spectrometer using neat thin film technique. High-resolution mass spectra (HRMS) were obtained using an Agilent 6224 Tof-MS spectrometer (ESI) and an Agilent 7200B QTof GC-MS spectrometer (EI) and are reported as calculated/observed $\mathrm{m} / \mathrm{z}$ for $[\mathrm{M}]^{+}$, $[\mathrm{M}+\mathrm{H}]^{+}$, or $[\mathrm{M}+\mathrm{Na}]^{+}$ions. Nuclear magnetic resonance spectra $\left({ }^{1} \mathrm{H}\right.$ NMR, ${ }^{13} \mathrm{C}$ NMR and ${ }^{19} \mathrm{~F}$ NMR) were obtained using a Bruker Model DMX $400\left(400 \mathrm{MHz}:{ }^{1} \mathrm{H}\right.$ at $400 \mathrm{MHz},{ }^{13} \mathrm{C}$ at $101 \mathrm{MHz},{ }^{19} \mathrm{~F}$ at $\left.376 \mathrm{MHz}\right)$; some NMR spectra $\left({ }^{1} \mathrm{H},{ }^{2} \mathrm{H},{ }^{13} \mathrm{C}\right)$ were obtained using a Bruker Model DMX $500\left(500 \mathrm{MHz}:{ }^{1} \mathrm{H}\right.$ at $500 \mathrm{MHz},{ }^{2} \mathrm{H}$ at $77 \mathrm{MHz},{ }^{13} \mathrm{C}$ at 126 $\mathrm{MHz}$ ). For $\mathrm{CDCl}_{3}$ solutions, the chemical shifts were reported as parts per million (ppm) referenced to residual protium or carbon of the solvents: $\mathrm{CDCl}_{3} \delta \mathrm{H}(7.26 \mathrm{ppm})$ and $\mathrm{CDCl}_{3} \delta \mathrm{C}(77.16 \mathrm{ppm})$. Coupling constants were reported in Hertz (Hz). Data for ${ }^{1} \mathrm{H}$ NMR spectra were reported as following: chemical shift $(\delta, \mathrm{ppm})$, multiplicity (br $=$ broad, $\mathrm{s}$ $=$ singlet, $\mathrm{d}=$ doublet, $\mathrm{t}=$ triplet, $\mathrm{q}=$ quartet, $\mathrm{dd}=$ doublet of doublets, $\mathrm{td}=$ triplet of doublets, $\mathrm{ddd}=$ doublet of doublet of doublets, $\mathrm{m}=$ multiplet), coupling constant $(\mathrm{Hz})$, and integration. Deuterium incorporation was assigned by ${ }^{1} \mathrm{H}$ or ${ }^{2} \mathrm{H}$ NMR analysis of the reaction products. The X-ray structure of $\mathbf{4 d}$ was obtained using a Bruker D8 VENTURE Single Crystal Dual-Source Diffractometer. All ortho-Heck products obtained were racemic; stereochemistry was shown to highlight that the products were single diastereomers.

\section{Synthesis \& characterization of substrates}

Compounds $1 \mathbf{a},{ }^{1} \mathbf{1} \mathbf{k},{ }^{2} \mathbf{1} \mathbf{s},{ }^{1,3} \mathbf{1} \mathbf{t},{ }^{4} \mathbf{2 e},{ }^{5}$ and $3 \mathbf{v}^{6}$ were prepared according to literature procedures. A diazotization procedure $^{7}$ was used to prepare compounds $\mathbf{1} \mathbf{j},{ }^{7} \mathbf{1 1},{ }^{8}$ and $\mathbf{1 0},{ }^{9}$ which all matched previously reported spectra. Isotopically labeled compound $\mathbf{1 i - d} 7$ is new and was prepared via a bromination ${ }^{10}$ procedure, and then converted to the aryl iodide; labeled acrylate $\mathbf{2 a - d}{ }^{11}$ is known. Compounds $\mathbf{1 i}, \mathbf{1 m}, \mathbf{1 n}, \mathbf{1 p}, \mathbf{1 q}, \mathbf{1 r}, \mathbf{2 a}, \mathbf{2 b}, \mathbf{2 c}, \mathbf{2 d}, \mathbf{2 f}, \mathbf{2 g}, \mathbf{2 h}$, and $3 \mathbf{u}$ are all commercially available.

Known compounds:<smiles>Cc1cc(N2CCOCC2)ccc1I</smiles>

$1 a$<smiles>Ic1ccc(N2CCOCC2)cc1</smiles>

1s<smiles>Ic1cccc2ncccc12</smiles>

1j<smiles>Cc1ccc2c(c1)c(I)cc(=[18O])n2Cc1ccccc1</smiles><smiles>Cc1cc(N(C)C)ccc1I</smiles>

$1 \mathrm{k}$<smiles>[2H]C([2H])=C([2H])C(=O)OC</smiles><smiles>COc1ccc(I)c(C)c1</smiles>

11<smiles>C=CC(=O)c1ccccc1</smiles>

$2 e$<smiles>Cc1cc(C#N)ccc1I</smiles>

10<smiles>O=C1[C@H]2[C@H]3C=C[C@@H](C3)[C@H]2C(=O)[NH+]1c1ccccc1</smiles>

$3 v$ 
Commercial compounds:<smiles>Ic1cccc2ccccc12</smiles>

$1 \mathrm{i}$<smiles>Fc1ccccc1I</smiles>

$1 r$<smiles>C=CC#N</smiles>

$2 f$<smiles>Cc1ccccc1I</smiles>

$1 \mathrm{~m}$<smiles>C=CC(=O)OC</smiles>

$2 a$<smiles>C=CS(C)(=O)=O</smiles>

2g<smiles>Cc1cc(Br)ccc1I</smiles>

1n<smiles>C=CC(=O)OCc1ccccc1</smiles>

2b<smiles>C=C[PH2+]</smiles>

$2 \mathrm{~h}$<smiles>COC(=O)c1ccc(I)c(C)c1</smiles>

$1 p$<smiles>C=CC(C)=O</smiles>

2c

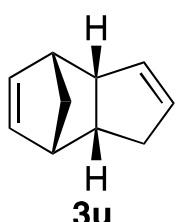<smiles>COc1ccccc1I</smiles>

$1 q$<smiles>C=CC(=O)NCCC</smiles>

2d<smiles>C1=CC2C=CC1C2</smiles>

$3 w$

New compound:<smiles>[2H]c1c([2H])c([2H])c2c(I)c([2H])c([2H])c([2H])c2c1[2H]</smiles>

$1 \mathrm{i}-\mathrm{d}_{7}$

\section{Labeled compounds:}<smiles>COC(=O)C([18OH])=C([18O])[18OH]</smiles>

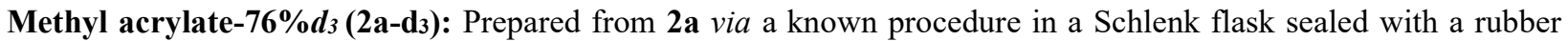
septum. ${ }^{11}$ After distillation, the labeled acrylate was found to contain $76 \%$ deuterium at all three acrylic positions. ${ }^{1} \mathbf{H}$ NMR $(500 \mathrm{MHz}$, Chloroform- $d$ ) $\delta 6.44-6.35(\mathrm{~m}, 0.24 \mathrm{H}), 6.16-6.08(\mathrm{~m}, 0.23 \mathrm{H}), 5.85-5.78(\mathrm{~m}, 0.25 \mathrm{H}), 3.76(\mathrm{~s}$, $3 \mathrm{H}) .{ }^{2} \mathrm{H}$ NMR $\left(77 \mathrm{MHz}, \mathrm{CDCl}_{3}\right) \delta 6.42(\mathrm{~s}, 1 \mathrm{H}), 6.15(\mathrm{~s}, 1 \mathrm{H}), 5.85(\mathrm{~s}, 1 \mathrm{H})$. Note: on a smaller scale, the high Dincorporations reported by Ackermann and co-workers ${ }^{11}$ was reproduced. On the larger scale used for material isolation, however, deuterium incorporations were reduced to $76 \%$ for uncertain reasons. 


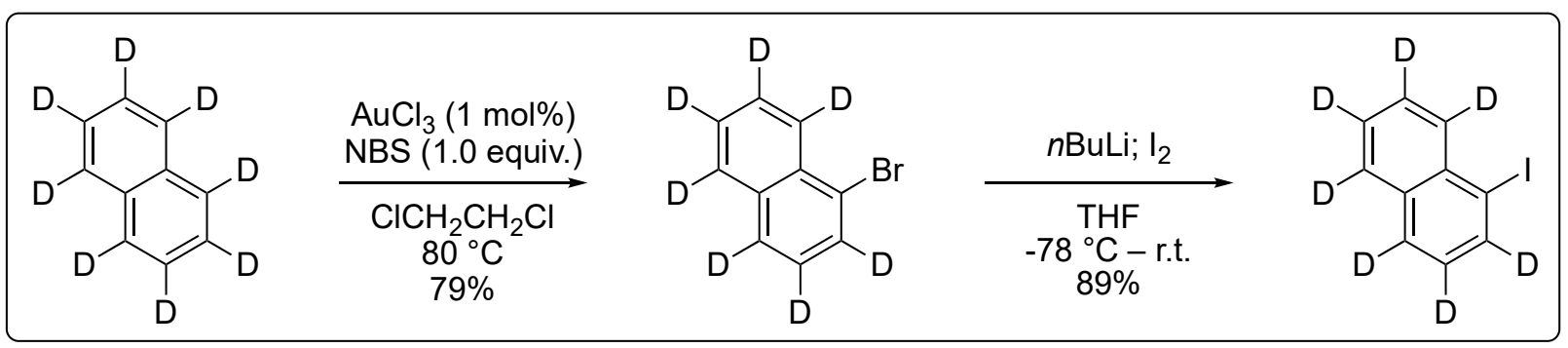

1-iodonaphthalene- $\boldsymbol{d}_{7}$ (1i-d7): Naphthalene- $d_{8}$ (1.0 equiv., $\left.5.0 \mathrm{mmol}, 681.1 \mathrm{mg}\right), \mathrm{AuCl}_{3}(0.01$ equiv., $0.05 \mathrm{mmol}$, $15.2 \mathrm{mg}), 1,2$-dichloroethane $(0.5 \mathrm{M}, 10.0 \mathrm{~mL})$, and $N$-bromosuccinimide (1.0 equiv., $5.0 \mathrm{mmol}, 889.9 \mathrm{mg}) \mathrm{were}$ added successively to a flame-dried $40 \mathrm{~mL}$ vial inside a nitrogen-filled glove box. The vial was sealed, brought outside the glove box, and heated at $80{ }^{\circ} \mathrm{C}$ overnight. The reaction was allowed to cool, was concentrated, and purified via silica gel chromatography to obtain $848.7 \mathrm{mg} \mathrm{(79 \% )}$ as a yellow oil, which matched the previously reported spectra. ${ }^{12}$ The aryl bromide (1.0 equiv., $3.74 \mathrm{mmol}, 800 \mathrm{mg}$ ) was then dissolved in dry THF $(0.1 \mathrm{M}, 37.4 \mathrm{~mL})$ inside a nitrogenfilled Schlenk flask and then cooled to $-78^{\circ} \mathrm{C}$. $n$ BuLi (1.1 equiv., $4.11 \mathrm{mmol}, 1.64 \mathrm{~mL} ; 2.5 \mathrm{M}$ in hexanes) was added dropwise and allowed to stir at $-78^{\circ} \mathrm{C}$ for $30 \mathrm{~min}$. Iodine ( 1.2 equiv., $4.48 \mathrm{mmol}, 1.14 \mathrm{~g}$ ) was then dissolved in minimal THF and added dropwise to the solution. The reaction was allowed to stir at room temperature for 6 hours, then quenched with sat. $\mathrm{NH}_{4} \mathrm{Cl}$, extracted with diethyl ether, washed with $10 \% \mathrm{Na}_{2} \mathrm{~S}_{2} \mathrm{O}_{3}$, dried over $\mathrm{MgSO}_{4}$, filtered, and concentrated. Pale yellow oil. 89\% yield $(869 \mathrm{mg}) . \mathrm{R}_{\mathrm{f}}=0.7$ (hexanes). ${ }^{2} \mathbf{H} \mathbf{N M R}\left(77 \mathrm{MHz}, \mathrm{CDCl}_{3}\right) \delta 8.15,7.91,7.84$, 7.64, 7.58, 7.54. ${ }^{13} \mathbf{C}$ NMR $\left(101 \mathrm{MHz}, \mathrm{CDCl}_{3}\right) \delta 137.3,137.1,136.8,134.4,134.1,133.4,132.0,131.7,131.5,128.9$, $128.6,128.4,128.2,127.9,127.7,127.5,127.3,127.1,126.7,126.5,126.4,126.3,126.2,126.1,125.6,125.4,125.2$, 99.4. IR (KBr, cm $\left.{ }^{-1}\right) 2289,2270,1536,1437,1250,902,624$. HRMS (EI) m/z: [M] ${ }^{+}$Calcd for C $10 \mathrm{D}_{7} \mathrm{I}: 261.0032$; Found: 261.0033 .

\section{Experimental procedures for the ortho-alkenylation reactions}

General Procedure for $\mathrm{Pd} / \mathrm{NBE}$ reactions: $\mathrm{Pd}(\mathrm{dba})_{2}, \mathrm{P}(\mathrm{fur})_{3}$, and base (if not moisture sensitive) were placed into a flame-dried vial with a stir bar. Solid aryl iodide $(0.1 \mathrm{mmol})$ was also added at this stage. The vial was sealed and

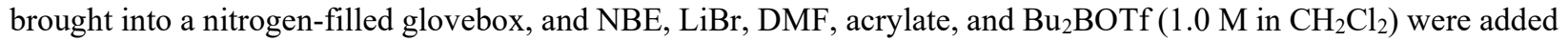
successively. If $\mathrm{Cs}_{2} \mathrm{CO}_{3}$ or a liquid aryl halide was used, it was added inside the glove box prior to the addition of $\mathrm{DMF}$. The reaction vial was sealed, removed from the glove box, and heated at $100^{\circ} \mathrm{C}$ for $18 \mathrm{~h}$ (note: the temperature was monitored via an alcohol thermometer submerged in a vial filled with silicone oil, not the hot plate’s temperature probe).

For test-scale reactions (0.1 $\mathbf{~ m m o l ) : ~ U p o n ~ c o m p l e t i o n , ~ t h e ~ r e a c t i o n s ~ w e r e ~ a l l o w e d ~ t o ~ c o o l ~ t o ~ r o o m ~ t e m p e r a t u r e , ~ w e r e ~}$ quenched by pouring onto water in a $13 \times 100 \mathrm{~mm}$ test tube, and extracted $4 \mathrm{x}$ with diethyl ether. The organic fractions were dried by filtering through an $\mathrm{MgSO}_{4}$ plug, concentrated, and placed under vacuum on a Schlenk line to remove residual solvent. The internal standard, 1,1,2,2-tetrachloroethane (16.8 $\mathrm{mg}, 0.1 \mathrm{mmol})$, was added to the crude residue, which was then diluted with $\mathrm{CDCl}_{3}$ and analyzed via crude ${ }^{1} \mathrm{H}$ NMR analysis to determine yield and composition.

For isolation-scale reactions $\mathbf{( 0 . 2} \mathbf{~ m m o l})$ : Upon completion, the reactions were allowed to cool to room temperature, and diluted with ethyl acetate. The reactions were quenched by pouring onto water, then extracted with diethyl ether. The organic fractions were dried by filtering through an $\mathrm{MgSO}_{4}$ plug, concentrated, and purified via silica gel chromatography (EtOAc/hexanes). Some compounds were further purified via preparatory TLC, and any impurities found have been accounted for in the isolated yields.

For the large-scale reaction $(\mathbf{1 . 0} \mathbf{~ m m o l})$ : Upon completion, the reaction was allowed to cool to room temperature, diluted with ethyl acetate, quenched by pouring onto water in a separatory funnel, and extracted with diethyl ether. The organics were dried over $\mathrm{MgSO}_{4}$, filtered, concentrated, and purified via silica gel chromatography (EtOAc/hexanes). 


\section{Selected reaction optimization and misc. examples}

Table S1. Misc. Reaction Optimization

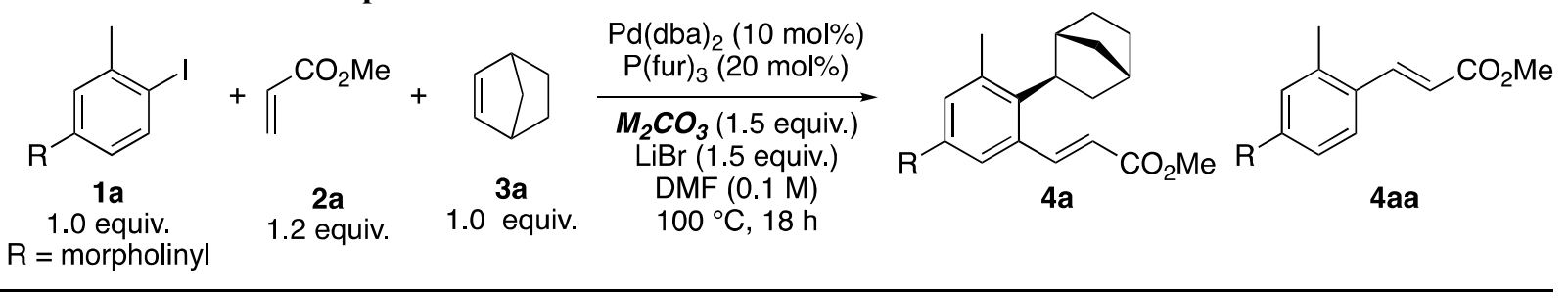

\begin{tabular}{|c|c|c|c|c|}
\hline & Carbonate salt & $\%$ 1a recovered & $\%$ yield $\mathbf{4 a}$ & $\%$ yield $\mathbf{4 a a}$ \\
\hline \multirow{4}{*}{ w/o $\mathrm{LiBr}$} & $\mathrm{Li}_{2} \mathrm{CO}_{3}$ & Not observed & $22 \%$ & $17 \%$ \\
\hline & $\mathrm{Na}_{2} \mathrm{CO}_{3}$ & Not observed & $15 \%$ & $10 \%$ \\
\hline & $\mathrm{K}_{2} \mathrm{CO}_{3}$ & Not observed & $23 \%$ & $16 \%$ \\
\hline & $\mathrm{Cs}_{2} \mathrm{CO}_{3}$ & Not observed & $1 \%$ & $2 \%$ \\
\hline \multirow{4}{*}{$\mathrm{w} / \mathrm{LiBr}$} & $\mathrm{Li}_{2} \mathrm{CO}_{3}$ & Not observed & $54 \%$ & $12 \%$ \\
\hline & $\mathrm{Na}_{2} \mathrm{CO}_{3}$ & Not observed & $49 \%$ & $13 \%$ \\
\hline & $\mathrm{K}_{2} \mathrm{CO}_{3}$ & Not observed & $61 \%$ & $8 \%$ \\
\hline & $\mathrm{Cs}_{2} \mathrm{CO}_{3}$ & Not observed & $44 \%$ & $13 \%$ \\
\hline
\end{tabular}

Scheme S1. Unsuccessful Substrates

R

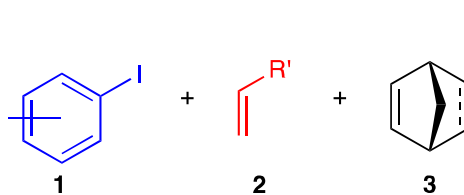

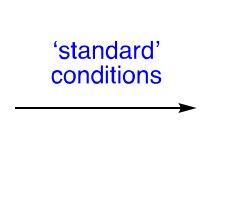

$\mathrm{R}$

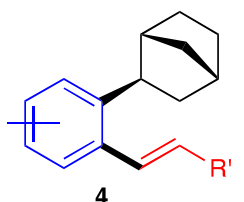

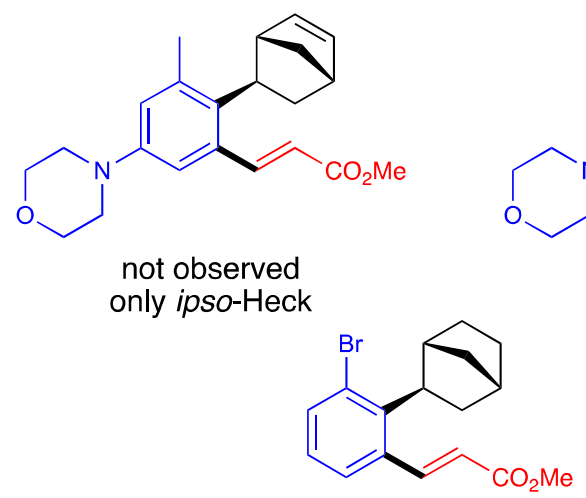

not observed<smiles>CC(=O)O/C=C/c1cc(N2CCOCC2)cc(C)c1[C@H]1C[C@@H]2CC[C@H]1C2</smiles>

not observed<smiles>CC(=O)C=Cc1ccccc1[C@H]1C[C@H]2CC[C@H]1C2</smiles>

$<5 \%$

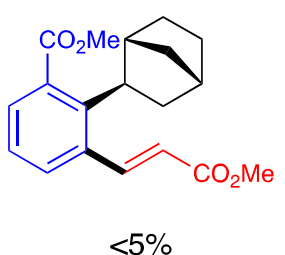

$<5 \%$ 
A moderate kinetic isotope effect has been observed:

Scheme S2. Intermolecular Competition KIE Experiment<smiles>IC1=CC(=[Tl])C=Cc2ccccc21</smiles>

( 0.5 equiv.)

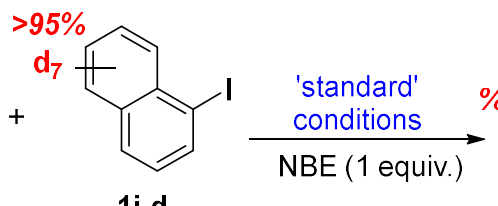

0.5 equiv $)$

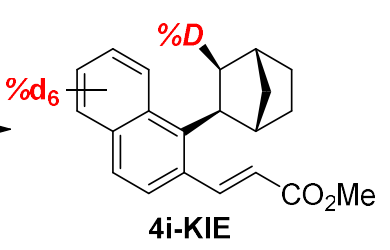

$\mathrm{t}=15 \mathrm{~min}: 19 \%$ yield, $40 \% \mathrm{D}$ $\mathrm{t}=30 \mathrm{~min}: 41 \%$ yield, $41 \% \mathrm{D}$

\section{Synthesis \& Characterization of Products}<smiles>COC(=O)/C=C/c1cc(N2CCOCC2)cc(C)c1[C@H]1C[C@H]2CC[C@H]1C2</smiles>

methyl (E)-3-(2-(bicyclo[2.2.1]heptan-2-yl)-3-methyl-5-morpholinophenyl)acrylate (4a): Synthesized from 1a, 2a, and 3a according to the general procedure. $0.2 \mathrm{mmol}$ scale: $69 \%$ yield $(48.8 \mathrm{mg}) ; 1.0 \mathrm{mmol}$ scale: $75 \%$ yield $(266.1$ mg). Yellow oil. $\mathrm{R}_{f}=0.36$ (hexane/EtOAc $\left.=4: 1\right) .{ }^{1} \mathbf{H}$ NMR $(400 \mathrm{MHz}$, Chloroform- $d) \delta 8.42(\mathrm{~d}, J=15.5 \mathrm{~Hz}, 1 \mathrm{H})$, $6.77(\mathrm{~d}, J=2.8 \mathrm{~Hz}, 1 \mathrm{H}), 6.71(\mathrm{~d}, J=2.8 \mathrm{~Hz}, 1 \mathrm{H}), 6.09(\mathrm{~d}, J=15.5 \mathrm{~Hz}, 1 \mathrm{H}), 3.88-3.82(\mathrm{~m}, 4 \mathrm{H}), 3.81(\mathrm{~s}, 3 \mathrm{H}), 3.16-$ $3.09(\mathrm{~m}, 4 \mathrm{H}), 2.96(\mathrm{t}, J=8.3 \mathrm{~Hz}, 1 \mathrm{H}), 2.57-2.53(\mathrm{~m}, 1 \mathrm{H}), 2.38(\mathrm{~s}, 3 \mathrm{H}), 2.36(\mathrm{~s}, 1 \mathrm{H}), 1.83(\mathrm{~m}, 2 \mathrm{H}), 1.66-1.58(\mathrm{~m}$, 2H), 1.54 (dddd, $J=11.9,7.5,3.7,2.2 \mathrm{~Hz}, 1 \mathrm{H}), 1.45-1.35(\mathrm{~m}, 1 \mathrm{H}), 1.35-1.17(\mathrm{~m}, 2 \mathrm{H}) .{ }^{13} \mathbf{C}$ NMR $(101 \mathrm{MHz}$, $\left.\mathrm{CDCl}_{3}\right) \delta 167.6,148.8,148.6,138.4,135.8,135.0,120.4,119.5,114.1,67.0,51.8,49.4,45.4,41.8,40.88,38.7,36.7$, 32.8, 28.6, 22.7. IR $\left(\mathrm{KBr}, \mathrm{cm}^{-1}\right)$ 2952, 2868, 1718, 1598, 1251, 1169, 1123, 992. HRMS (ESI) m/z: [M+Na] ${ }^{+}$Calcd for $\mathrm{C}_{22} \mathrm{H}_{29} \mathrm{NO}_{3} \mathrm{Na}$ : 378.2045 ; Found: 378.2047 .<smiles>CO/C=C/c1ccc(N2CCOCC2)cc1C</smiles>

methyl (E)-3-(2-methyl-4-morpholinophenyl)acrylate (4aa): Palladium(II) acetate (0.05 equiv., 0.05 mmol, 11.2 $\mathrm{mg}$ ), triphenylphosphine ( 0.1 equiv., $0.1 \mathrm{mmol}, 26.2 \mathrm{mg}$ ), and aryl iodide $1 \mathrm{a}$ (1.0 equiv., $1.0 \mathrm{mmol}, 303 \mathrm{mg}$ ) were placed in a flame-dried $20 \mathrm{~mL}$ vial and transferred into a nitrogen-filled glovebox. Triethylamine $(0.2 \mathrm{M}, 5.0 \mathrm{~mL})$ and acrylate $2 \mathrm{a}$ ( 1.3 equiv., $1.3 \mathrm{mmol}, 120 \mu \mathrm{L}$ ) were added successively, then the vial was sealed and heated at $90{ }^{\circ} \mathrm{C}$ overnight. The reaction was then allowed to cool to room temperature, concentrated, and purified via silica gel chromatography. $88 \%$ (229.8 mg). Tan solid. Melting point: $103-105^{\circ} \mathrm{C} . \mathrm{R}_{f}=0.29$ (hexane/EtOAc $\left.=4: 1\right) .{ }^{1} \mathbf{H}$ NMR $(400 \mathrm{MHz}$, Chloroform- $d$ ) $\delta 7.91(\mathrm{~d}, J=15.8 \mathrm{~Hz}, 1 \mathrm{H}), 7.52(\mathrm{~d}, J=8.7 \mathrm{~Hz}, 1 \mathrm{H}), 6.74(\mathrm{dd}, J=8.7,2.7 \mathrm{~Hz}, 1 \mathrm{H}), 6.69$ $(\mathrm{d}, J=2.7 \mathrm{~Hz}, 1 \mathrm{H}), 6.25(\mathrm{~d}, J=15.8 \mathrm{~Hz}, 1 \mathrm{H}), 3.88-3.83(\mathrm{~m}, 4 \mathrm{H}), 3.79(\mathrm{~s}, 3 \mathrm{H}), 3.26-3.18(\mathrm{~m}, 4 \mathrm{H}) .{ }^{13} \mathbf{C}$ NMR $(101$ $\left.\mathrm{MHz}, \mathrm{CDCl}_{3}\right) \delta 167.7,148.3,143.9,137.4,134.3,132.7,127.4,125.8,119.6,51.8,46.3,41.7,40.7,38.9,36.7,32.9$, 28.7, 22.3. IR $\left(\mathrm{KBr}, \mathrm{cm}^{-1}\right) 2952$, 2851, 1709, 1598, 1240, 1165. HRMS (ESI) m/z: $[\mathrm{M}+\mathrm{Na}]^{+}$Calcd for $\mathrm{C}_{15} \mathrm{H}_{19} \mathrm{NO}_{3} \mathrm{Na}$ : 284.1263; Found: 284.1265 . The ${ }^{1} \mathrm{H}$ NMR spectrum matches the spectrum obtained from the proposed ipso-Heck side product isolated from the reaction mixtures in Table 1. 


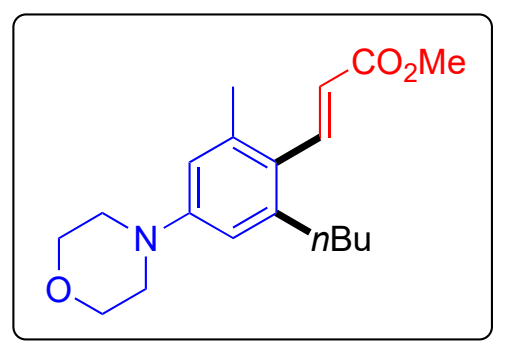

methyl (E)-3-(2-butyl-6-methyl-4-morpholinophenyl)acrylate (4ab): Synthesized according to similar conditions reported by Catellani \& co-workers. ${ }^{14} \mathrm{Pd}(\mathrm{OAc})_{2}(20 \mathrm{~mol} \%, 4.5 \mathrm{mg}), \mathrm{K}_{2} \mathrm{CO}_{3}$ (5.3 equiv., $\left.73.3 \mathrm{mg}\right), \mathrm{KOAc}(5.3 \mathrm{equiv}$., $52.0 \mathrm{mg}$ ), and aryl iodide 1a (1.0 equiv., $30.3 \mathrm{mg}$ ) were placed in a flame-dried vial equipped with a magnetic stirbar, which was then loosely capped and transferred to a nitrogen-filled glove box. Norbornene (2.0 equiv., $18.8 \mathrm{mg})$ and DMF (0.075 M, $1.33 \mathrm{~mL})$, followed by methyl acrylate (1.0 equiv., $8.6 \mathrm{mg})$, and $n \mathrm{BuI}(6.67 \mathrm{equiv} ., 122.7 \mathrm{mg}) \mathrm{in}$ that order. The vial was sealed and heated at $55^{\circ} \mathrm{C}$ or $100^{\circ} \mathrm{C}$ for $18 \mathrm{~h}$. The general procedure for test-scale reactions was then followed. $31 \%$ yield $\left(55^{\circ} \mathrm{C}\right)$ and $41 \%$ yield $\left(100^{\circ} \mathrm{C}\right)$. Note: NMR yields were obtained because while 4 ab is unreported, it is not the focus of this manuscript. $\mathrm{R}_{f}=0.38$ (hexane/EtOAc $=17: 3$ ). ${ }^{1} \mathbf{H} \mathbf{~ N M R}(400 \mathrm{MHz}$, Chloroform- $d) \delta 7.88(\mathrm{~d}, J=16.3 \mathrm{~Hz}, 1 \mathrm{H}), 6.60(\mathrm{~s}, 2 \mathrm{H}), 6.02(\mathrm{~d}, J=16.3 \mathrm{~Hz}, 1 \mathrm{H}), 3.89-3.82(\mathrm{~m}, 4 \mathrm{H}), 3.80(\mathrm{~s}, 3 \mathrm{H})$, $3.26-3.12(\mathrm{~m}, 4 \mathrm{H}), 2.71-2.60(\mathrm{~m}, 2 \mathrm{H}), 2.36(\mathrm{~s}, 3 \mathrm{H}), 1.56-1.49(\mathrm{~m}, 2 \mathrm{H}), 1.37(\mathrm{q}, J=7.4 \mathrm{~Hz}, 2 \mathrm{H}), 0.92(\mathrm{t}, J=7.3$ $\mathrm{Hz}, 3 \mathrm{H}) .{ }^{13} \mathbf{C}$ NMR $\left(101 \mathrm{MHz}, \mathrm{CDCl}_{3}\right) \delta 167.7,148.3,143.9,137.4,134.3,132.7,127.4,125.8,119.6,51.8,46.3$, 41.7, 40.7, 38.9, 36.7, 32.9, 28.7, 22.3. IR (KBr, $\left.\mathrm{cm}^{-1}\right)$ 2955, 2926, 2856, 1716, 1598, 1310, 1259, 1161, 1123, 989, 881. HRMS (ESI) m/z: [M+Na $]^{+}$Calcd for $\mathrm{C}_{19} \mathrm{H}_{27} \mathrm{NO}_{3} \mathrm{Na}$ : 340.1889; Found: 340.1882. This product was not observed in entry 12 of Table 1.

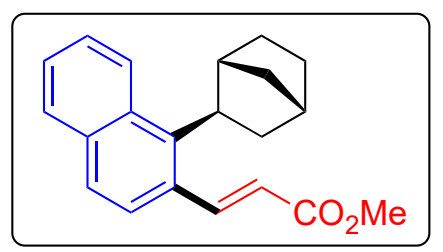

methyl (E)-3-(1-(bicyclo[2.2.1]heptan-2-yl)naphthalen-2-yl)acrylate (4i): Synthesized from 1i, 2a, and 3a according to the general procedure. $0.2 \mathrm{mmol}$ scale: $55 \%$ yield $(42.7 \mathrm{mg})$. Colorless oil. $\mathrm{R}_{f}=0.25$ (hexane/EtOAc $=$ 19:1). ${ }^{1} \mathbf{H}$ NMR (400 MHz, Chloroform- $d$ ) $\delta 8.73(\mathrm{~d}, J=15.7 \mathrm{~Hz}, 1 \mathrm{H}), 8.34-8.28(\mathrm{~m}, 1 \mathrm{H}), 7.80(\mathrm{dd}, J=7.9,1.7 \mathrm{~Hz}$, $1 \mathrm{H}), 7.66(\mathrm{~d}, J=8.5 \mathrm{~Hz}, 1 \mathrm{H}), 7.56-7.45(\mathrm{~m}, 2 \mathrm{H}), 7.43(\mathrm{~d}, J=8.6 \mathrm{~Hz}, 1 \mathrm{H}), 6.24(\mathrm{~d}, J=15.7 \mathrm{~Hz}, 1 \mathrm{H}), 3.84(\mathrm{~s}, 3 \mathrm{H})$, $3.67(\mathrm{t}, J=8.5 \mathrm{~Hz}, 1 \mathrm{H}), 2.90-2.85(\mathrm{~m}, 1 \mathrm{H}), 2.44(\mathrm{~s}, 1 \mathrm{H}), 2.19-2.10(\mathrm{~m}, 1 \mathrm{H}), 1.95(\mathrm{dp}, J=10.0,2.0 \mathrm{~Hz}, 1 \mathrm{H}), 1.74$ $(\mathrm{dtt}, J=9.1,3.9,2.4 \mathrm{~Hz}, 2 \mathrm{H}), 1.71-1.65(\mathrm{~m}, 1 \mathrm{H}), 1.56(\mathrm{ddq}, J=10.0,3.0,1.5 \mathrm{~Hz}, 1 \mathrm{H}), 1.51-1.44(\mathrm{~m}, 2 \mathrm{H}) .{ }^{13} \mathrm{C}$ NMR $\left(101 \mathrm{MHz} \mathrm{CDCl}_{3}\right) \delta 167.7,147.9,142.1,134.6,132.8,129.8,128.9,127.0,126.6,126.4,126.2,125.9,120.1$, 51.8, 46.0, 42.8, 42.1, 39.2, 36.9, 32.8, 29.0. IR (KBr, cm $\left.{ }^{-1}\right)$ 3055, 2950, 2869, 1718, 1627, 1192, 1173, 814. HRMS (ESI) $\mathrm{m} / \mathrm{z}:[\mathrm{M}+\mathrm{H}]^{+}$Calcd for $\mathrm{C}_{21} \mathrm{H}_{22} \mathrm{O}_{2} \mathrm{H}: 307.1698$; Found: 307.1693 .

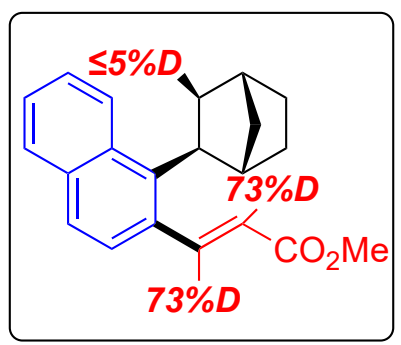

methyl $(E)-3-(1-((1 S, 2 S, 4 R)-b i c y c l o[2.2 .1] h e p t a n-2-y l) n a p h t h a l e n-2-y l) a c r y l a t e-d_{2}\left(4 i-d_{2}\right)$ : Synthesized from $1 \mathrm{i}$, 2a-d 3 , and 3a according to the general procedure. $0.1 \mathrm{mmol}$ scale: $47 \%$ yield (NMR). Colorless oil. $\mathrm{R}_{f}=0.25$ $($ hexane/EtOAc $=19: 1){ }^{1} \mathbf{H}$ NMR $(400 \mathrm{MHz}$, Chloroform- $d) \delta 8.76-8.69(\mathrm{~m}, 0.27 \mathrm{H}), 8.30(\mathrm{~d}, J=8.4 \mathrm{~Hz}, 1 \mathrm{H}), 7.80$ $(\mathrm{dd}, J=7.8,1.8 \mathrm{~Hz}, 1 \mathrm{H}), 7.66(\mathrm{~d}, J=8.6 \mathrm{~Hz}, 1 \mathrm{H}), 7.56-7.41(\mathrm{~m}, 4 \mathrm{H}), 6.28-6.18(\mathrm{~m}, 0.27 \mathrm{H}), 3.84(\mathrm{~s}, 3 \mathrm{H}), 3.66(\mathrm{t}$, $J=8.4 \mathrm{~Hz}, 1 \mathrm{H}), 2.87(\mathrm{~d}, J=2.4 \mathrm{~Hz}, 1 \mathrm{H}), 2.44(\mathrm{~s}, 1 \mathrm{H}), 2.14(\mathrm{ddd}, J=12.0,9.3,2.3 \mathrm{~Hz}, 1 \mathrm{H}), 1.95(\mathrm{dp}, J=10.1,2.0$ 
$\mathrm{Hz}, 1 \mathrm{H}), 1.80-1.71(\mathrm{~m}, 2 \mathrm{H}), 1.71-1.64(\mathrm{~m}, 1 \mathrm{H}), 1.55(\mathrm{dt}, J=2.9,1.3 \mathrm{~Hz}, 1 \mathrm{H}), 1.51-1.45(\mathrm{~m}, 2 \mathrm{H}) .{ }^{2} \mathbf{H}$ NMR $(77$ MHz, Chloroform- $d) \delta 8.77(\mathrm{~s}, 1 \mathrm{H}), 6.27(\mathrm{~s}, 1 \mathrm{H})$.

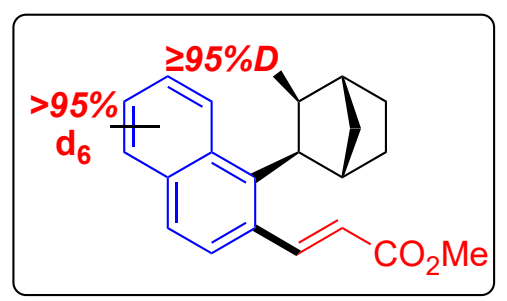

methyl (E)-3-(1-((1S,2S,3S,4R)-bicyclo[2.2.1] heptan-2-yl-3-d)naphthalen-2-yl-3,4,5,6,7,8-d6)acrylate (4i-d 7$)$ : Synthesized from 1i-d $7,2 \mathbf{2 a}$, and 3a according to the general procedure. $0.1 \mathrm{mmol}$ scale: See Scheme 3 for yields (NMR). Colorless oil. $\mathrm{R}_{f}=0.25$ (hexane/EtOAc $\left.=19: 1\right) .{ }^{1} \mathbf{H}$ NMR $(400 \mathrm{MHz}$, Chloroform- $d) \delta 8.72(\mathrm{~d}, J=15.6 \mathrm{~Hz}$, $1 \mathrm{H}), 6.23(\mathrm{~d}, J=15.7 \mathrm{~Hz}, 1 \mathrm{H}), 3.84(\mathrm{~s}, 4 \mathrm{H}), 3.65(\mathrm{~d}, J=9.3 \mathrm{~Hz}, 1 \mathrm{H}), 2.87(\mathrm{~d}, J=3.5 \mathrm{~Hz}, 1 \mathrm{H}), 2.43(\mathrm{~s}, 1 \mathrm{H}), 2.15-$ $2.07(\mathrm{~m}, 1 \mathrm{H}), 1.94(\mathrm{dt}, J=10.0,2.1 \mathrm{~Hz}, 1 \mathrm{H}), 1.77-1.70(\mathrm{~m}, 2 \mathrm{H}), 1.59-1.53(\mathrm{~m}, 1 \mathrm{H}), 1.47(\mathrm{dq}, J=9.3,2.5 \mathrm{~Hz}, 2 \mathrm{H})$. ${ }^{2}$ H NMR (77 MHz, Chloroform- $d$ ) $\delta 8.36(\mathrm{~s}, 1 \mathrm{H}), 7.97-7.42(\mathrm{~m}, 5 \mathrm{H}), 1.70(\mathrm{~s}, 1 \mathrm{H})$. HRMS (ESI) m/z: [M+Na] ${ }^{+}$ Calcd for $\mathrm{C}_{21} \mathrm{H}_{15} \mathrm{D}_{7} \mathrm{O}_{2} \mathrm{Na}: 336.1957$; Found: 336.1955 .

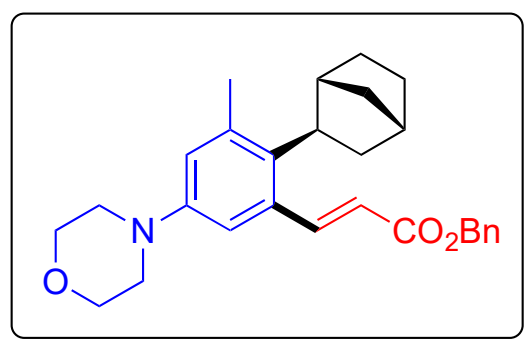

(E)-3-(2- benzyl (E)- 3-(2-(bicyclo[2.2.1] heptan-2-yl)-3-methyl-5-morpholinophenyl)acrylate (4b): Synthesized from 1a, $\mathbf{2 b}$, and $\mathbf{3 a}$ according to the general procedure. $0.2 \mathrm{mmol}$ scale: $73 \%$ yield $(62.9 \mathrm{mg})$. Yellow oil. $\mathrm{R}_{f}=0.38$ (hexane/EtOAc = 4:1). ${ }^{1} \mathbf{H}$ NMR $(400 \mathrm{MHz}$, Chloroform- $d$ ) $\delta 8.48(\mathrm{~d}, J=15.5 \mathrm{~Hz}, 1 \mathrm{H}), 7.46-7.32(\mathrm{~m}, 5 \mathrm{H}), 6.77(\mathrm{~d}$, $J=2.8 \mathrm{~Hz}, 1 \mathrm{H}), 6.72(\mathrm{~d}, J=2.7 \mathrm{~Hz}, 1 \mathrm{H}), 6.15(\mathrm{~d}, J=15.5 \mathrm{~Hz}, 1 \mathrm{H}), 5.26(\mathrm{~s}, 2 \mathrm{H}), 3.90-3.83(\mathrm{~m}, 4 \mathrm{H}), 3.17-3.09$ (m, $3 \mathrm{H}), 2.96(\mathrm{t}, J=8.3 \mathrm{~Hz}, 1 \mathrm{H}), 2.55(\mathrm{~s}, 1 \mathrm{H}), 2.38(\mathrm{~s}, 3 \mathrm{H}), 2.34(\mathrm{~s}, 1 \mathrm{H}), 1.90-1.73(\mathrm{~m}, 2 \mathrm{H}), 1.71-1.50(\mathrm{~m}, 2 \mathrm{H}), 1.40$ $-1.26(\mathrm{~m}, 3 \mathrm{H}) .{ }^{13} \mathbf{C}$ NMR $\left(101 \mathrm{MHz}, \mathrm{CDCl}_{3}\right) \delta 166.9,149.1,148.6,138.5,136.3,135.9,134.8,128.7,128.4,128.4$, 120.4, 119.5, 114.0, 67.0, 66.4, 49.4, 45.5, 41.8, 40.9, 38.8, 36.7, 32.9, 28.7, 22.8. IR (KBr, cm $\left.{ }^{-1}\right) 3032,2954,2867$, 1713, 1598, 1160. HRMS (ESI) m/z: [M+H] ${ }^{+}$Calcd for $\mathrm{C}_{28} \mathrm{H}_{33} \mathrm{NO}_{3} \mathrm{H}$; 432.2539. Found: 432.2542 .

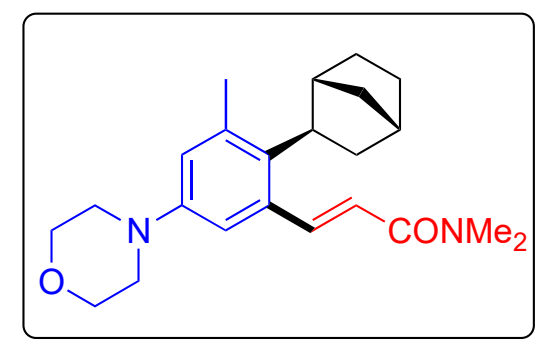

(E)-O-(3-(2-(bicyclo[2.2.1] heptan-2-yl)-3-methyl-5-morpholinophenyl)acryloyl)- $N, N$-dimethylhydroxylamine (4c): Synthesized from 1a, 2c, and 3a according to the general procedure. $0.2 \mathrm{mmol}$ scale: $59 \%$ yield $(43.4 \mathrm{mg}$ ). Viscous dark red oil. $\mathrm{R}_{f}=0.22$ (hexane/EtOAc $\left.=2: 3\right) .{ }^{1} \mathbf{H}$ NMR $(400 \mathrm{MHz}$, Chloroform- $d) \delta 8.32(\mathrm{dd}, J=15.1,1.8$ $\mathrm{Hz}, 1 \mathrm{H}), 6.73(\mathrm{~d}, J=2.9 \mathrm{~Hz}, 1 \mathrm{H}), 6.67(\mathrm{~d}, J=2.7 \mathrm{~Hz}, 1 \mathrm{H}), 6.47(\mathrm{dd}, J=15.1,1.4 \mathrm{~Hz}, 1 \mathrm{H}), 3.84(\mathrm{dq}, J=5.1,2.2 \mathrm{~Hz}$, 4H), $3.18-3.09$ (m, 7H), 3.07 (s, 3H), 2.95 (m, 1H), 2.55 (s, 1H), 2.38 (d, $J=1.8 \mathrm{~Hz}, 3 \mathrm{H}), 2.33$ (s, 1H), 1.82 (ddd, $J$ $=12.0,9.6,2.4 \mathrm{~Hz}, 2 \mathrm{H}), 1.63-1.53(\mathrm{~m}, 3 \mathrm{H}), 1.45-1.23(\mathrm{~m}, 5 \mathrm{H}) .{ }^{13} \mathbf{C} \mathbf{~ N M R}\left(101 \mathrm{MHz}, \mathrm{CDCl}_{3}\right) \delta 166.8,148.5,146.6$, 138.2, 136.4, 135.7, 119.8, 119.5, 114.6, 67.0, 49.6, 45.5, 41.8, 40.7, 38.7, 37.5, 36.6, 36.0, 32.7, 28.7, 22.8. IR (KBr, $\mathrm{cm}^{-1}$ ) 3380, 2953, 2868, 2241, 1647, 1600, 1394, 1122, 731. HRMS (ESI) m/z: [M+Na] Calcd for $\mathrm{C}_{23} \mathrm{H}_{32} \mathrm{~N}_{2} \mathrm{O}_{2} \mathrm{Na}$ : 391.2361; Found: 391.2356. 


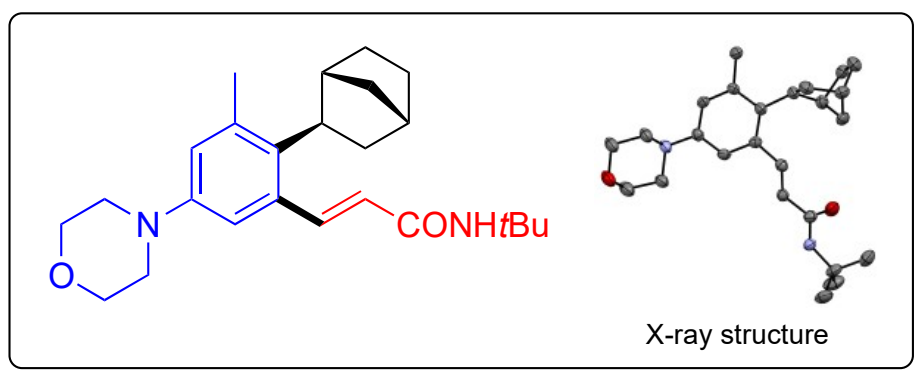

(bicyclo[2.2.1] heptan-2-yl)-3-methyl-5-morpholinophenyl)- $\mathbf{N}$-(tert-butyl)acryl-amide (4d): Synthesized from 1a, 2d, and 3a according to the general procedure. $0.2 \mathrm{mmol}$ scale: $88 \%$ yield $(69.5 \mathrm{mg})$. Pale-yellow solid. Decomposition point: $183-188^{\circ} \mathrm{C} . \mathrm{R}_{f}=0.28$ (hexane/EtOAc $=4: 1$ ). A single crystal was obtained by vapor diffusion of pentane into a solution of $\mathbf{4 d}$ dissolved in ethyl acetate, which was analyzed via X-ray crystallography. ${ }^{1} \mathbf{H}$ NMR (400 MHz, Chloroform- $d) \delta 8.20(\mathrm{~d}, J=15.0 \mathrm{~Hz}, 1 \mathrm{H}), 6.72(\mathrm{~d}, J=2.8 \mathrm{~Hz}, 1 \mathrm{H}), 6.66(\mathrm{~d}, J=2.8 \mathrm{~Hz}, 1 \mathrm{H}), 5.92(\mathrm{~d}, J=15.1 \mathrm{~Hz}$, $1 \mathrm{H}), 5.36(\mathrm{~s}, 1 \mathrm{H}), 3.88-3.79(\mathrm{~m}, 4 \mathrm{H}), 3.16-3.08(\mathrm{~m}, 4 \mathrm{H}), 2.95(\mathrm{t}, J=8.3 \mathrm{~Hz}, 1 \mathrm{H}), 2.58-2.54(\mathrm{~m}, 1 \mathrm{H}), 2.38(\mathrm{~s}, 3 \mathrm{H})$, $2.34(\mathrm{~s}, 1 \mathrm{H}), 1.83$ (tdt, $J=7.2,5.4,2.3 \mathrm{~Hz}, 2 \mathrm{H}), 1.63-1.50(\mathrm{~m}, 4 \mathrm{H}), 1.43(\mathrm{~s}, 9 \mathrm{H}), 1.33-1.25(\mathrm{~m}, 2 \mathrm{H}) .{ }^{13} \mathbf{C} \mathbf{N M R}$ $\left(101 \mathrm{MHz}, \mathrm{CDCl}_{3}\right) \delta 165.3,148.5,143.9,138.1,135.9,135.7,124.1,119.8,114.4,67.1,51.5,49.5,45.5,41.7,40.8$, 38.8, 36.7, 32.8, 29.1, 28.7, 22.8. HRMS (ESI) m/z: [M+H] ${ }^{+}$Calcd for $\mathrm{C}_{25} \mathrm{H}_{36} \mathrm{~N}_{2} \mathrm{O} 2 \mathrm{H}: 397.2855$; Found: 397.2857.

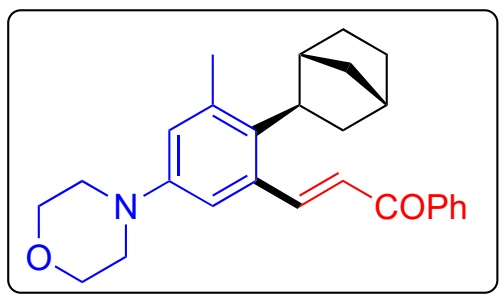

(E)-3-(2-(bicyclo[2.2.1]heptan-2-yl)-3-methyl-5-morpholinophenyl)-1-phenylprop-2-en-1-one (4e): Synthesized from 1a, 2e, and 3a according to the general procedure. $0.2 \mathrm{mmol} \mathrm{scale:} 57 \%$ yield (46.1 mg). Yellow oil. $\mathrm{R}_{f}=0.32$ (hexane/EtOAc = 4:1). ${ }^{1} \mathbf{H}$ NMR $(400 \mathrm{MHz}$, Chloroform-d) $\delta 8.52(\mathrm{~d}, J=15.2 \mathrm{~Hz}, 1 \mathrm{H}), 8.04-7.98(\mathrm{~m}, 2 \mathrm{H}), 7.61-$ $7.55(\mathrm{~m}, 1 \mathrm{H}), 7.53-7.48(\mathrm{~m}, 2 \mathrm{H}), 7.16(\mathrm{~d}, J=15.2 \mathrm{~Hz}, 1 \mathrm{H}), 6.82(\mathrm{~d}, J=2.8 \mathrm{~Hz}, 1 \mathrm{H}), 6.80(\mathrm{~d}, J=2.8 \mathrm{~Hz}, 1 \mathrm{H}), 3.90$ $-3.82(\mathrm{~m}, 4 \mathrm{H}), 3.18-3.13(\mathrm{~m}, 4 \mathrm{H}), 2.99(\mathrm{t}, J=8.4 \mathrm{~Hz}, 1 \mathrm{H}), 2.56(\mathrm{~s}, 1 \mathrm{H}), 2.40(\mathrm{~s}, 3 \mathrm{H}), 2.33(\mathrm{~s}, 2 \mathrm{H}), 1.88-1.81(\mathrm{~m}$, $1 \mathrm{H}), 1.78(\mathrm{~d}, J=10.5 \mathrm{~Hz}, 1 \mathrm{H}), 1.64-1.57(\mathrm{~m}, 3 \mathrm{H}), 1.38-1.28(\mathrm{~m}, 3 \mathrm{H}) .{ }^{13} \mathbf{C ~ N M R}\left(101 \mathrm{MHz}, \mathrm{CDCl}_{3}\right) \delta 190.8,149.1$, $148.7,138.5,138.3,136.5,135.7,132.8,128.8,128.6,128.4,124.2,120.6,114.2,67.1,49.5,45.6,41.9,40.9,38.7$, 36.7, 32.8, 28.7, 22.8. IR (KBr, cm $\left.{ }^{-1}\right)$ 2953, 2866, 1662, 1600, 1448, 1253, 1122, 1016, 694. HRMS (ESI) m/z: $[\mathrm{M}+\mathrm{Na}]^{+}$Calcd for $\mathrm{C}_{27} \mathrm{H}_{31} \mathrm{NO}_{2} \mathrm{Na}$ : 424.2252; Found: 424.2253 .

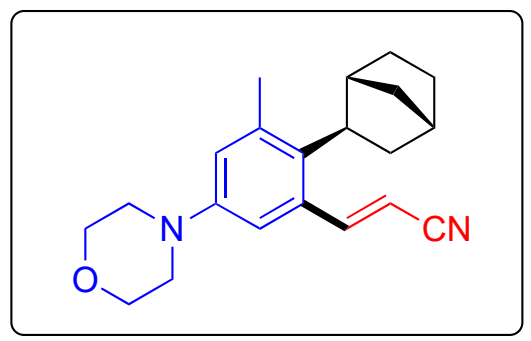

(E)-3-(2-(bicyclo[2.2.1]heptan-2-yl)-3-methyl-5-morpholinophenyl)acrylonitrile (4f): Synthesized from 1a, 2f, and 3a according to the general procedure. $0.2 \mathrm{mmol}$ scale: $88 \%$ yield $(56.5 \mathrm{mg} ; E: Z=1.35: 1)$. Yellow oil. $\mathrm{R}_{f}=0.4$ (hexane/EtOAc = 4:1). ${ }^{1} \mathbf{H}$ NMR $(500 \mathrm{MHz}$, Chloroform- $d) \delta 8.15(\mathrm{~d}, J=16.2 \mathrm{~Hz}, 0.56 \mathrm{H}), 7.85(\mathrm{~d}, J=11.6 \mathrm{~Hz}$, $0.43 \mathrm{H}), 6.86(\mathrm{~d}, J=2.8 \mathrm{~Hz}, 0.48 \mathrm{H}), 6.78(\mathrm{dd}, J=6.0,2.8 \mathrm{~Hz}, 1 \mathrm{H}), 6.57(\mathrm{~d}, J=2.8 \mathrm{~Hz}, 0.56 \mathrm{H}), 5.55(\mathrm{~d}, J=16.2 \mathrm{~Hz}$, $0.52 \mathrm{H}), 5.47(\mathrm{~d}, J=11.5 \mathrm{~Hz}, 0.39 \mathrm{H}), 3.85(\mathrm{t}, J=5.1 \mathrm{~Hz}, 4 \mathrm{H}), 3.14(\mathrm{~m}, 4 \mathrm{H}), 2.91(\mathrm{~m}, 1 \mathrm{H}), 2.55-2.49(\mathrm{~m}, 1 \mathrm{H}), 2.37$ $(\mathrm{s}, 3 \mathrm{H}), 2.33(\mathrm{~s}, 0.69 \mathrm{H}), 1.86(\mathrm{dtd}, J=11.3,8.9,2.3 \mathrm{~Hz}, 1 \mathrm{H}), 1.72-1.57(\mathrm{~m}, 3 \mathrm{H}), 1.50-1.39(\mathrm{~m}, 1 \mathrm{H}), 1.37-1.23$ $(\mathrm{m}, 3 \mathrm{H}) .{ }^{13} \mathbf{C}$ NMR $\left(101 \mathrm{MHz}, \mathrm{CDCl}_{3}\right) \delta 154.7,154.0,148.7,138.7,138.5,135.4,134.1,133.3,121.1,120.8,118.4$, 117.3 , 115.7, 113.6, 98.1, 97.6, 67.0, 66.9, 49.5, 49.3, 45.5, 41.6, 41.5, 41.0, 40.7, 38.8, 36.6, 36.5, 32.7, 32.6, 28.5, 
22.8, 22.7. IR $\left(\mathrm{KBr}, \mathrm{cm}^{-1}\right)$ 2955, 2868, 2216, 1597, 1450, 1258, 1122. HRMS (ESI) m/z: $[\mathrm{M}+\mathrm{Na}]^{+}$Calcd for $\mathrm{C}_{21} \mathrm{H}_{26} \mathrm{~N}_{2} \mathrm{ONa}$ : 345.1943: Found: 345.1944.

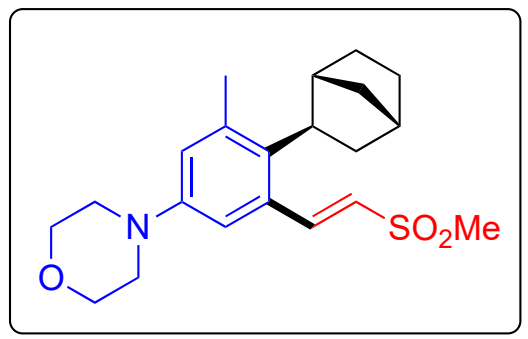

(E)-4-(4-(bicyclo[2.2.1]heptan-2-yl)-3-methyl-5-(2-(methylsulfonyl)vinyl)phenyl)morpholine (4g): Synthesized from $1 \mathrm{a}, \mathbf{2 g}$, and 3a according to the general procedure. $0.2 \mathrm{mmol}$ scale: $83 \%$ yield $(62.0 \mathrm{mg})$. Dark yellow solid. Decomposition point: $179-183{ }^{\circ} \mathrm{C} . \mathrm{R}_{f}=0.12$ (hexane/EtOAc $\left.=1: 1\right) .{ }^{1} \mathbf{H}$ NMR $(400 \mathrm{MHz}$, Chloroform- $d$ ) $\delta 8.40(\mathrm{~d}$, $J=15.1 \mathrm{~Hz}, 1 \mathrm{H}), 6.80(\mathrm{~d}, J=2.8 \mathrm{~Hz}, 1 \mathrm{H}), 6.63(\mathrm{~d}, J=2.8 \mathrm{~Hz}, 1 \mathrm{H}), 6.58(\mathrm{~d}, J=15.0 \mathrm{~Hz}, 1 \mathrm{H}), 3.87-3.82(\mathrm{~m}, 4 \mathrm{H})$, $3.15-3.10(\mathrm{~m}, 4 \mathrm{H}), 3.02(\mathrm{~s}, 3 \mathrm{H}), 2.99-2.91(\mathrm{~m}, 1 \mathrm{H}), 2.54(\mathrm{~s}, 1 \mathrm{H}), 2.38(\mathrm{~s}, 3 \mathrm{H}), 2.37(\mathrm{~s}, 1 \mathrm{H}), 2.17(\mathrm{~s}, 3 \mathrm{H}), 1.88(\mathrm{td}$, $J=10.3,9.1,2.2 \mathrm{~Hz}, 1 \mathrm{H}), 1.73(\mathrm{~d}, J=10.1 \mathrm{~Hz}, 1 \mathrm{H}), 1.63(\mathrm{~d}, J=9.4 \mathrm{~Hz}, 2 \mathrm{H}), 1.35-1.27(\mathrm{~m}, 2 \mathrm{H}) .{ }^{13} \mathbf{C}$ NMR $(101$ $\left.\mathrm{MHz}, \mathrm{CDCl}_{3}\right) \delta 148.8,148.1,138.9,136.2,132.3,127.5,121.1,114.0,67.0,49.4,45.6,43.2,41.7,41.0,38.8,36.7$, 32.7, 28.7, 22.7. IR $\left(\mathrm{KBr}, \mathrm{cm}^{-1}\right) 2955,2867,1596,1451,1305,1131,964,834,506$. HRMS (ESI) m/z: $[\mathrm{M}+\mathrm{H}]^{+}$Calcd for $\mathrm{C}_{21} \mathrm{H}_{29} \mathrm{NO}_{3} \mathrm{SH}$ : 376.1946; Found: 376.1942 .

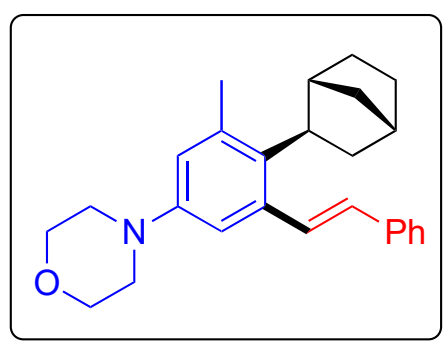

(E)-4-(4-(bicyclo[2.2.1]heptan-2-yl)-3-methyl-5-styrylphenyl)morpholine (4h): Synthesized from 1a, 2h, and 3a according to the general procedure. $0.1 \mathrm{mmol}$ scale: $20 \%$ yield (NMR). An NMR yield was obtained for $\mathbf{4 h}$ because separation from unreacted 1a via silica gel chromatography proved to be quite challenging. Colorless oil. $\mathrm{R}_{f}=0.25$ (hexane/EtOAc = 9:1). ${ }^{1} \mathbf{H}$ NMR $(400 \mathrm{MHz}$, Chloroform- $d) \delta 7.72(\mathrm{~d}, J=15.9 \mathrm{~Hz}, 1 \mathrm{H}), 7.51-7.44(\mathrm{~m}, 2 \mathrm{H}), 7.36$ $(\mathrm{dd}, J=8.4,6.7 \mathrm{~Hz}, 2 \mathrm{H}), 6.80(\mathrm{~d}, J=2.8 \mathrm{~Hz}, 1 \mathrm{H}), 6.71(\mathrm{~d}, J=2.8 \mathrm{~Hz}, 1 \mathrm{H}), 6.65(\mathrm{~d}, J=15.9 \mathrm{~Hz}, 1 \mathrm{H}), 3.92-3.70(\mathrm{~m}$, 4H), $3.27-3.01(\mathrm{~m}, 4 \mathrm{H}), 3.00(\mathrm{t}, J=8.3 \mathrm{~Hz}, 1 \mathrm{H}), 2.63(\mathrm{~s}, 1 \mathrm{H}), 2.39(\mathrm{~s}, 3 \mathrm{H}), 2.33(\mathrm{~s}, 1 \mathrm{H}), 1.91-1.79(\mathrm{~m}, 2 \mathrm{H}), 1.71$ $-1.59(\mathrm{~m}, 4 \mathrm{H}), 1.37-1.27(\mathrm{~m}, 3 \mathrm{H}) .{ }^{13} \mathbf{C}$ NMR $\left(126 \mathrm{MHz}, \mathrm{CDCl}_{3}\right) \delta 148.7,138.1,138.0,138.0,134.8,132.1,130.2$, 128.9, 127.5, 126.5, 118.7, 114.6, 67.2, 49.7, 45.6, 41.8, 40.5, 38.8, 36.7, 32.8, 28.8, 22.9. IR $\left(\mathrm{KBr}, \mathrm{cm}^{-1}\right) 3023,2953$, 2853, 1597, 1449, 1261, 1123, 692. HRMS (ESI) m/z: [M+H] ${ }^{+}$Calcd for $\mathrm{C}_{26} \mathrm{H}_{31} \mathrm{NOH}$ : 374.2484; Found: 374.2481.

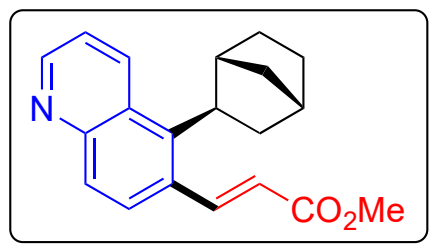

methyl (E)-3-(5-(bicyclo[2.2.1] heptan-2-yl)quinolin-6-yl)acrylate (4j): Synthesized from 1j, 2a, and 3a according to the general procedure. $0.2 \mathrm{mmol}$ scale: $49 \%$ yield $\left(29.8 \mathrm{mg}\right.$ ). Viscous yellow oil. $\mathrm{R}_{f}=0.15$ (hexane/EtOAc $\left.=4: 1\right)$. ${ }^{1}$ H NMR (400 MHz, Chloroform- $d$ ) $\delta 8.88(\mathrm{dt}, J=3.9,1.9 \mathrm{~Hz}, 1 \mathrm{H}), 8.69-8.65(\mathrm{~m}, 1 \mathrm{H}), 8.64(\mathrm{~s}, 1 \mathrm{H}), 7.92(\mathrm{dd}, J=$ $8.9,2.3 \mathrm{~Hz}, 1 \mathrm{H}), 7.65(\mathrm{dd}, J=8.8,2.0 \mathrm{~Hz}, 1 \mathrm{H}), 7.43(\mathrm{ddd}, J=9.1,4.1,1.9 \mathrm{~Hz}, 1 \mathrm{H}), 6.25$ (dd, $J=15.7,2.1 \mathrm{~Hz}, 1 \mathrm{H})$, $3.84(\mathrm{~s}, 3 \mathrm{H}), 3.58(\mathrm{t}, J=8.4 \mathrm{~Hz}, 1 \mathrm{H}), 2.87(\mathrm{~d}, J=3.0 \mathrm{~Hz}, 1 \mathrm{H}), 2.44(\mathrm{~s}, 1 \mathrm{H}), 2.08$ (ddd, $J=12.0,9.3,2.3 \mathrm{~Hz}, 1 \mathrm{H}), 1.90$ $(\mathrm{dp}, J=10.0,2.0 \mathrm{~Hz}, 1 \mathrm{H}), 1.75-1.71(\mathrm{~m}, 2 \mathrm{H}), 1.71-1.61(\mathrm{~m}, 1 \mathrm{H}), 1.56(\mathrm{dq}, J=10.1,1.7 \mathrm{~Hz}, 1 \mathrm{H}), 1.48-1.24(\mathrm{~m}$, 
2H). ${ }^{13}$ C NMR (101 MHz, $\left.\mathrm{CDCl}_{3}\right) \delta 167.5,150.2,149.4,146.8,142.3,134.2,130.5,130.4,128.3,127.9,121.1,121.1$, 51.9, 45.8, 42.9, 41.9, 39.1, 36.9, 32.6, 28.9. HRMS (ESI) m/z: $[\mathrm{M}+\mathrm{Na}]^{+}$Calcd for $\mathrm{C}_{20} \mathrm{H}_{21} \mathrm{NO}_{2} \mathrm{Na}$ : 330.1470 ; Found: 330.1469 .

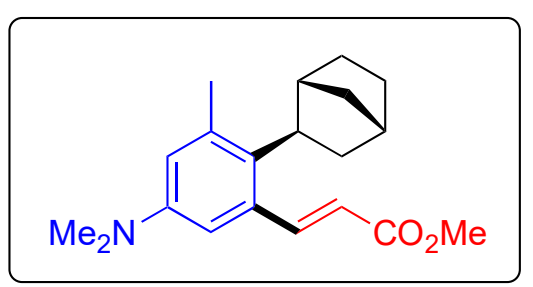

methyl (E)-3-(2-(bicyclo[2.2.1] heptan-2-yl)-5-(dimethylamino)-3-methylphenyl)acrylate (4k): Synthesized from $1 \mathbf{k}, 2 \mathbf{a}$, and 3a according to the general procedure. $0.2 \mathrm{mmol}$ scale: $70 \%$ yield $\left(44.1 \mathrm{mg}\right.$ ). Yellow oil. $\mathrm{R}_{f}=0.47$ (hexane/EtOAc = 9:1). ${ }^{1} \mathbf{H}$ NMR $(400 \mathrm{MHz}$, Chloroform- $d) \delta 8.43(\mathrm{~d}, J=15.5 \mathrm{~Hz}, 1 \mathrm{H}), 6.62(\mathrm{~d}, J=2.9 \mathrm{~Hz}, 1 \mathrm{H}), 6.54$ $(\mathrm{d}, J=2.9 \mathrm{~Hz}, 1 \mathrm{H}), 6.12(\mathrm{~d}, J=15.5 \mathrm{~Hz}, 1 \mathrm{H}), 3.81(\mathrm{~s}, 3 \mathrm{H}), 2.95(\mathrm{t}, J=8.3 \mathrm{~Hz}, 1 \mathrm{H}), 2.91(\mathrm{~s}, 6 \mathrm{H}), 2.56-2.51(\mathrm{~m}, 1 \mathrm{H})$, $2.38(\mathrm{~s}, 3 \mathrm{H}), 2.35(\mathrm{q}, J=3.1,2.2 \mathrm{~Hz}, 1 \mathrm{H}), 1.83(\mathrm{tdd}, J=9.0,5.7,2.3 \mathrm{~Hz}, 2 \mathrm{H}), 1.65-1.58(\mathrm{~m}, 3 \mathrm{H}), 1.37-1.28(\mathrm{~m}$, $3 \mathrm{H}) .{ }^{13} \mathrm{C}$ NMR $\left(101 \mathrm{MHz}, \mathrm{CDCl}_{3}\right) \delta 167.8,149.4,148.2,138.2,134.9,132.5,119.2,117.6,111.2,51.7,45.2,41.9$, 41.0, 40.8, 38.7, 36.8, 32.9, 28.7, 22.8. IR (KBr, cm $\left.{ }^{-1}\right)$ 2949, 2868, 1716, 1601, 1304, 1168, 837. HRMS (ESI) m/z: $[\mathrm{M}+\mathrm{Na}]^{+}$Calcd for $\mathrm{C}_{20} \mathrm{H}_{27} \mathrm{NO}_{2} \mathrm{Na}$ : 336.1939; Found: 336.1935 .

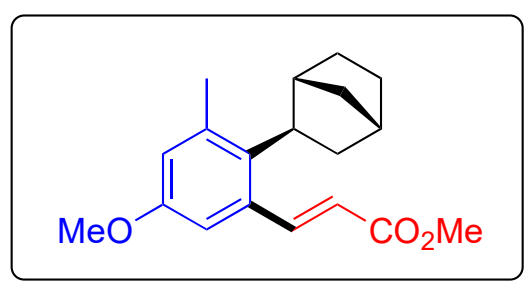

methyl (E)-3-(2-(bicyclo[2.2.1]heptan-2-yl)-5-methoxy-3-methylphenyl)acrylate (4I): Synthesized from 11, 2a, and $3 \mathbf{a}$ according to the general procedure. $0.2 \mathrm{mmol}$ scale: $62 \%$ yield $\left(38.9 \mathrm{mg}\right.$ ). Yellow oil. $\mathrm{R}_{f}=0.65$ (hexane/EtOAc = 9:1). ${ }^{1} \mathbf{H}$ NMR (400 MHz, Chloroform- $d$ ) $\delta 8.42(\mathrm{~d}, J=15.5 \mathrm{~Hz}, 1 \mathrm{H}), 6.75(\mathrm{~d}, J=2.9 \mathrm{~Hz}, 1 \mathrm{H}), 6.72(\mathrm{~d}, J=2.9 \mathrm{~Hz}$, $1 \mathrm{H}), 6.11(\mathrm{~d}, J=15.6 \mathrm{~Hz}, 1 \mathrm{H}), 3.81(\mathrm{~s}, 3 \mathrm{H}), 3.78(\mathrm{~s}, 3 \mathrm{H}), 2.97(\mathrm{t}, J=8.4 \mathrm{~Hz}, 1 \mathrm{H}), 2.58-2.53(\mathrm{~m}, 1 \mathrm{H}), 2.38(\mathrm{~s}, 3 \mathrm{H})$, $2.36(\mathrm{~d}, J=5.4 \mathrm{~Hz}, 1 \mathrm{H}), 1.90-1.79(\mathrm{~m}, 2 \mathrm{H}), 1.61(\mathrm{dq}, J=5.9,3.4 \mathrm{~Hz}, 2 \mathrm{H}), 1.54$ (dddd, $J=11.8,7.6,3.8,2.3 \mathrm{~Hz}$, $1 \mathrm{H}), 1.40(\mathrm{ddq}, J=10.0,2.9,1.5 \mathrm{~Hz}, 1 \mathrm{H}), 1.32(\mathrm{dq}, J=9.2,2.1 \mathrm{~Hz}, 2 \mathrm{H}) .{ }^{13} \mathbf{C}$ NMR $\left(101 \mathrm{MHz}, \mathrm{CDCl}_{3}\right) \delta 167.6,156.8$, $148.2,139.0,136.5,135.2,119.7,118.8,111.5,55.3,51.8,45.5,41.8,40.9,38.8,36.7,32.9,28.6,22.6$. IR $\left(\mathrm{KBr}, \mathrm{cm}^{-}\right.$ 1) $2951,2869,2091,1716,1630,1600,1471,1435,1169,1069,859$. HRMS (ESI) m/z: [M+H] ${ }^{+}$Calcd for $\mathrm{C}_{19} \mathrm{H}_{24} \mathrm{O}_{3} \mathrm{H}$ : 301.1804; Found: 301.1803 .

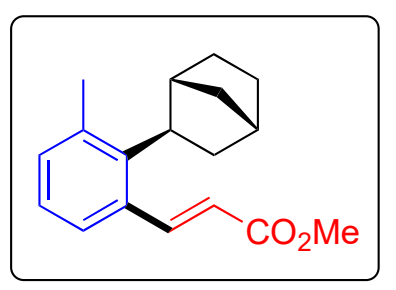

methyl (E)-3-(2-(bicyclo[2.2.1]heptan-2-yl)-3-methylphenyl)acrylate $(4 \mathrm{~m})$ : Synthesized from $1 \mathrm{~m}$, 2a, and 3a according to the general procedure. $0.2 \mathrm{mmol}$ scale: $43 \%$ yield $\left(23.3 \mathrm{mg}\right.$ ). Colorless oil. $\mathrm{R}_{f}=0.31$ (hexane $/$ EtOAc $=$ 19:1). ${ }^{1} \mathrm{H}$ NMR (400 MHz, Chloroform- $d$ ) $\delta 8.44(\mathrm{~d}, J=15.6 \mathrm{~Hz}, 1 \mathrm{H}), 7.18$ (ddd, $\left.J=11.6,7.8,4.1 \mathrm{~Hz}, 2 \mathrm{H}\right), 7.06(\mathrm{t}$, $J=7.5 \mathrm{~Hz}, 1 \mathrm{H}), 6.10(\mathrm{~d}, J=15.5 \mathrm{~Hz}, 1 \mathrm{H}), 3.81(\mathrm{~s}, 3 \mathrm{H}), 3.04(\mathrm{t}, J=8.4 \mathrm{~Hz}, 1 \mathrm{H}), 2.61(\mathrm{~d}, J=2.7 \mathrm{~Hz}, 1 \mathrm{H}), 2.41(\mathrm{~s}, 3 \mathrm{H})$, $2.37(\mathrm{~s}, 1 \mathrm{H}), 1.93-1.81(\mathrm{~m}, 3 \mathrm{H}), 1.69-1.58(\mathrm{~m}, 3 \mathrm{H}), 1.37-1.29(\mathrm{~m}, 3 \mathrm{H}) .{ }^{13} \mathbf{C} \mathbf{~ N M R}\left(101 \mathrm{MHz}, \mathrm{CDCl}_{3}\right) \delta 167.7$, 148.3, 143.9, 137.4, 134.3, 132.7, 127.4, 125.8, 119.6, 51.8, 46.3, 41.7, 40.7, 38.9, 36.7, 32.9, 28.7, 22.3. IR (KBr, $\mathrm{cm}^{-1}$ ) 2950, 2869, 1719, 1629, 1163, 792. HRMS (ESI) m/z: $[\mathrm{M}+\mathrm{H}]^{+}$Calcd for $\mathrm{C}_{18} \mathrm{H}_{22} \mathrm{O}_{2} \mathrm{H}$ : 271.1698; Found: 271.1693. 


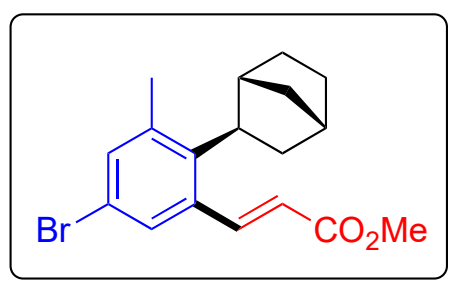

methyl (E)-3-(2-(bicyclo[2.2.1]heptan-2-yl)-5-bromo-3-methylphenyl)acrylate (4n): Synthesized from 1n, 2a, and 3a according to the general procedure. $0.2 \mathrm{mmol}$ scale: $61 \%$ yield (42.6 mg). Pale-yellow oil. $\mathrm{R}_{f}=0.63$ (hexane/EtOAc $=19: 1) .{ }^{1} \mathbf{H}$ NMR $(400 \mathrm{MHz}$, Chloroform- $d) \delta 8.33(\mathrm{~d}, J=15.5 \mathrm{~Hz}, 1 \mathrm{H}), 7.30(\mathrm{~d}, J=2.3 \mathrm{~Hz}, 1 \mathrm{H})$, $7.28(\mathrm{~d}, J=2.4 \mathrm{~Hz}, 1 \mathrm{H}), 6.09(\mathrm{~d}, J=15.5 \mathrm{~Hz}, 1 \mathrm{H}), 3.81(\mathrm{~s}, 3 \mathrm{H}), 2.95(\mathrm{t}, J=8.4 \mathrm{~Hz}, 1 \mathrm{H}), 2.60-2.55(\mathrm{~m}, 1 \mathrm{H}), 2.37(\mathrm{~s}$, $3 \mathrm{H}), 2.36(\mathrm{~d}, J=3.0 \mathrm{~Hz}, 1 \mathrm{H}), 1.92-1.82(\mathrm{~m}, 1 \mathrm{H}), 1.78(\mathrm{dp}, J=10.0,1.9 \mathrm{~Hz}, 1 \mathrm{H}), 1.68-1.59$ (m, $2 \mathrm{H}), 1.50(\mathrm{dddd}$, $J=11.7,7.5,3.8,2.3 \mathrm{~Hz}, 1 \mathrm{H}), 1.41$ (ddt, $J=10.0,4.0,1.5 \mathrm{~Hz}, 1 \mathrm{H}), 1.31$ (dt, $J=8.8,2.6 \mathrm{~Hz}, 2 \mathrm{H}) .{ }^{13} \mathbf{C}$ NMR $(101$ $\left.\mathrm{MHz}_{2} \mathrm{CDCl}_{3}\right) \delta 167.7,148.3,143.9,137.4,134.3,132.7,127.4,125.8,119.6,51.8,46.3,41.7,40.7,38.9,36.7,32.9$, 28.7, 22.3. IR (KBr, cm $\left.{ }^{-1}\right)$ 2951, 2870, 1721, 1632, 1309, 1170. HRMS (ESI) m/z: [M+H] $]^{+} \mathrm{Calcd}_{\text {for }} \mathrm{C}_{18} \mathrm{H}_{21} \mathrm{BrO}_{2} \mathrm{H}$ : 349.0803; Found: 349.0797.

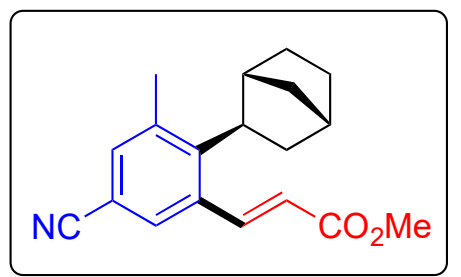

methyl (E)-3-(2-(bicyclo[2.2.1]heptan-2-yl)-5-cyano-3-methylphenyl)acrylate (4o): Synthesized from 10, 2a, and $3 \mathbf{a}$ according to the general procedure. $0.2 \mathrm{mmol} \mathrm{scale:} 45 \%$ yield $(26.6 \mathrm{mg})$. Yellow oil. $\mathrm{R}_{f}=0.46$ (hexane $/$ EtOAc $=$ 10:1). ${ }^{1} \mathbf{H}$ NMR $(400 \mathrm{MHz}$, Chloroform- $d) \delta 8.33(\mathrm{~d}, J=15.6 \mathrm{~Hz}, 1 \mathrm{H}), 7.43(\mathrm{~d}, J=2.0 \mathrm{~Hz}, 1 \mathrm{H}), 7.41(\mathrm{~d}, J=2.0 \mathrm{~Hz}$, $1 \mathrm{H}), 6.10(\mathrm{~d}, J=15.6 \mathrm{~Hz}, 1 \mathrm{H}), 3.82(\mathrm{~s}, 3 \mathrm{H}), 3.03(\mathrm{t}, J=8.4 \mathrm{~Hz}, 1 \mathrm{H}), 2.43(\mathrm{~s}, 3 \mathrm{H}), 2.39(\mathrm{~d}, J=4.2 \mathrm{~Hz}, 1 \mathrm{H}), 1.92(\mathrm{ddd}$, $J=11.9,9.1,2.3 \mathrm{~Hz}, 1 \mathrm{H}), 1.76(\mathrm{dp}, J=10.3,2.0 \mathrm{~Hz}, 1 \mathrm{H}), 1.70-1.61(\mathrm{~m}, 2 \mathrm{H}), 1.54-1.48(\mathrm{~m}, 1 \mathrm{H}), 1.47-1.42(\mathrm{~m}$, $2 \mathrm{H}), 1.36$ - $1.29(\mathrm{~m}, 2 \mathrm{H}) .{ }^{13} \mathbf{C}$ NMR $\left(101 \mathrm{MHz} \mathrm{CDCl}_{3}\right) \delta 167.7,148.3,143.9,137.4,134.3,132.7,127.4,125.8$, 119.6, 51.8, 46.3, 41.7, 40.7, 38.9, 36.7, 32.9, 28.7, 22.3. IR (KBr, cm $\left.{ }^{-1}\right)$ 2959, 2870, 2228, 1719, 1636, 1320, 1261, 1031, 800. HRMS (ESI) m/z: [M+H] ${ }^{+}$Calcd for $\mathrm{C}_{19} \mathrm{H}_{21} \mathrm{NO}_{2} \mathrm{H}: 296.1651$; Found: 296.1649.

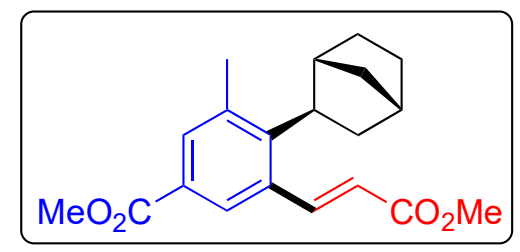

methyl (E)-4-(bicyclo[2.2.1]heptan-2-yl)-3-(3-methoxy-3-oxoprop-1-en-1-yl)-5-methylbenzo-ate (4p): Synthesized from 1p, 2a, and 3a according to the general procedure. 0.2 mmol scale: $28 \%$ yield (18.5 mg). Yellow oil. $\mathrm{R}_{f}=0.46($ hexane/EtOAc $=10: 1) .{ }^{1} \mathbf{H}$ NMR $(400 \mathrm{MHz}$, Chloroform- $d) \delta 8.40(\mathrm{~d}, J=15.6 \mathrm{~Hz}, 1 \mathrm{H}), 7.84(\mathrm{~d}, J=$ $2.0 \mathrm{~Hz}, 1 \mathrm{H}), 7.80(\mathrm{~d}, J=2.0 \mathrm{~Hz}, 1 \mathrm{H}), 6.18(\mathrm{~d}, J=15.6 \mathrm{~Hz}, 1 \mathrm{H}), 3.90(\mathrm{~s}, 3 \mathrm{H}), 3.82(\mathrm{~s}, 3 \mathrm{H}), 3.06(\mathrm{t}, J=8.4 \mathrm{~Hz}, 1 \mathrm{H})$, $2.64(\mathrm{~s}, 1 \mathrm{H}), 2.45(\mathrm{~s}, 3 \mathrm{H}), 2.38(\mathrm{~s}, 1 \mathrm{H}), 1.92(\mathrm{ddd}, J=11.8,8.8,2.1 \mathrm{~Hz}, 1 \mathrm{H}), 1.81(\mathrm{dt}, J=10.1,2.0 \mathrm{~Hz}, 1 \mathrm{H}), 1.64(\mathrm{dq}$, $J=8.5,3.4 \mathrm{~Hz}, 2 \mathrm{H}), 1.55-1.49(\mathrm{~m}, 1 \mathrm{H}), 1.48-1.41(\mathrm{~m}, 1 \mathrm{H}), 1.34(\mathrm{dt}, J=8.6,2.5 \mathrm{~Hz}, 2 \mathrm{H}) .{ }^{13} \mathrm{C} \mathrm{NMR}(101 \mathrm{MHz}$, $\left.\mathrm{CDCl}_{3}\right) \delta 167.7,148.3,143.9,137.4,134.3,132.7,127.4,125.8,119.6,51.8,46.3,41.7,40.7,38.9,36.7,32.9,28.7$, 22.3. IR $\left(\mathrm{KBr}, \mathrm{cm}^{-1}\right)$ 2954, 2360, 2342, 1720, 1261, 1034. HRMS (ESI) m/z: $[\mathrm{M}+\mathrm{H}]^{+}$Calcd for $\mathrm{C}_{20} \mathrm{H}_{24} \mathrm{O}_{4} \mathrm{H}: 329.1753$; Found: 329.1750 . 


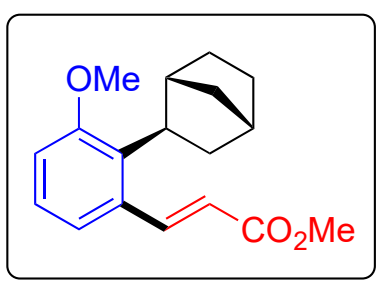

methyl (E)-3-(2-(bicyclo[2.2.1]heptan-2-yl)-3-methoxyphenyl)acrylate (4q): Synthesized from 1q, 2a, and 3a according to the general procedure. $0.2 \mathrm{mmol}$ scale: $37 \%$ yield $(21.4 \mathrm{mg})$. Yellow oil. $\mathrm{R}_{f}=0.35$ (hexane/EtOAc $=$ 10:1). ${ }^{1} \mathbf{H}$ NMR $(400 \mathrm{MHz}$, Chloroform- $d) \delta 8.17(\mathrm{~d}, J=15.7 \mathrm{~Hz}, 1 \mathrm{H}), 7.14(\mathrm{t}, J=7.9 \mathrm{~Hz}, 1 \mathrm{H}), 7.02(\mathrm{dd}, J=7.8,1.3$ $\mathrm{Hz}, 1 \mathrm{H}), 6.89(\mathrm{dd}, J=8.2,1.3 \mathrm{~Hz}, 1 \mathrm{H}), 6.20(\mathrm{~d}, J=15.7 \mathrm{~Hz}, 1 \mathrm{H}), 3.81(\mathrm{~s}, 3 \mathrm{H}), 3.78(\mathrm{~s}, 3 \mathrm{H}), 2.95(\mathrm{t}, J=8.2 \mathrm{~Hz}, 1 \mathrm{H})$, 2.49 (dt, $J=3.4,1.6 \mathrm{~Hz}, 1 \mathrm{H}), 2.31(\mathrm{~d}, J=4.2 \mathrm{~Hz}, 1 \mathrm{H}), 1.99(\mathrm{dt}, J=9.4,2.0 \mathrm{~Hz}, 1 \mathrm{H}), 1.85-1.77$ (m, $1 \mathrm{H}), 1.71$ (ddd, $J=13.3,8.8,2.2 \mathrm{~Hz}, 1 \mathrm{H}), 1.62-1.59(\mathrm{~m}, 1 \mathrm{H}), 1.57-1.52(\mathrm{~m}, 1 \mathrm{H}), 1.31(\mathrm{dddd}, J=13.0,8.7,5.0,2.0 \mathrm{~Hz}, 2 \mathrm{H}), 1.24$ $(\mathrm{dt}, J=8.9,1.7 \mathrm{~Hz}, 1 \mathrm{H}) .{ }^{13} \mathbf{C}$ NMR $\left(101 \mathrm{MHz}, \mathrm{CDCl}_{3}\right) \delta 167.7,148.3,143.9,137.4,134.3,132.7,127.4,125.8,119.6$, 51.8, 46.3, 41.7, 40.7, 38.9, 36.7, 32.9, 28.7, 22.3. IR (KBr, cm $\left.{ }^{-1}\right)$ 2949, 2869, 1719, 1469, 1256, 1166, 794. HRMS (ESI) $\mathrm{m} / \mathrm{z}:[\mathrm{M}+\mathrm{H}]^{+}$Calcd for $\mathrm{C}_{18} \mathrm{H}_{22} \mathrm{O}_{3} \mathrm{H}: 287.1647$; Found: 287.1645.

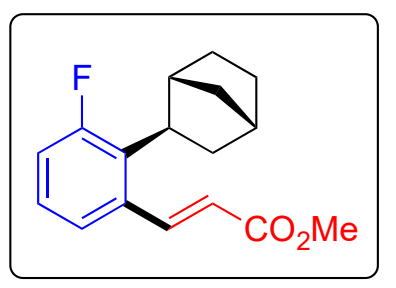

methyl (E)-3-(2-(bicyclo[2.2.1]heptan-2-yl)-3-fluorophenyl)acrylate (4r): Synthesized from 1r, 2a, and 3a according to the general procedure. $0.2 \mathrm{mmol}$ scale: $30 \%$ yield $\left(1: 1.3 \mathrm{4r}: 4 \mathrm{ra} ; 19.4 \mathrm{mg}\right.$ total). Yellow oil. $\mathrm{R}_{f}=0.62$ (hexane/EtOAc $=10: 1)$. Note: Products 4r and 4ra could not be separated by silica gel chromatography and were found to be unstable under preparatory TLC conditions. Therefore, the purity is limited and ${ }^{13} \mathrm{C}$ NMR spectra could not be obtained. ${ }^{1} \mathbf{H}$ NMR $(400 \mathrm{MHz}$, Chloroform- $d) \delta 8.06(\mathrm{~d}, J=15.7 \mathrm{~Hz}, 1 \mathrm{H}), 7.23(\mathrm{~s}, 1 \mathrm{H}), 7.12(\mathrm{tt}, J=8.2,4.0$ $\mathrm{Hz}, 1 \mathrm{H}), 7.00(\mathrm{dd}, J=12.5,7.9 \mathrm{~Hz}, 1 \mathrm{H}), 6.26(\mathrm{~d}, J=15.7 \mathrm{~Hz}, 1 \mathrm{H}), 3.82(\mathrm{~s}, 3 \mathrm{H}), 2.97-2.90(\mathrm{~m}, 1 \mathrm{H}), 2.61(\mathrm{~s}, 1 \mathrm{H})$, $2.34(\mathrm{~s}, 2 \mathrm{H}), 1.80$ (q, $J=14.1,13.4 \mathrm{~Hz}, 3 \mathrm{H}), 1.73-1.58(\mathrm{~m}, 8 \mathrm{H}), 1.35-1.29(\mathrm{~m}, 6 \mathrm{H}) .{ }^{19} \mathbf{F}$ NMR (376 MHz, Chloroform- $d) \delta-111.54(\mathrm{~d}, J=12.5 \mathrm{~Hz})$. IR $\left(\mathrm{KBr}, \mathrm{cm}^{-1}\right)$ 2951, 2870, 1722, 1636, 1464, 1314, $12311172,982,796$. HRMS (ESI) m/z: [M+H] ${ }^{+}$Calcd for $\mathrm{C}_{17} \mathrm{H}_{19} \mathrm{FO}_{2} \mathrm{H}$ : 275.1447; Found: 275.1445 .

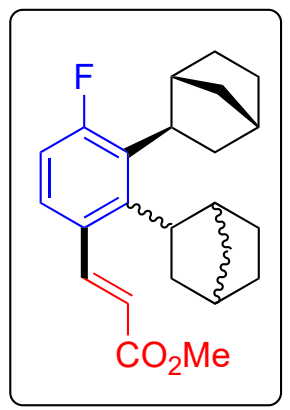

methyl (E)-3-(2,3-di(bicyclo[2.2.1]heptan-2-yl)-4-fluorophenyl)acrylate (4ra): Synthesized from 1r, 2a, and 3a according to the general procedure. $0.2 \mathrm{mmol}$ scale: $30 \%$ yield (1:1.3 4r:4ra; $19.4 \mathrm{mg}$ total). Yellow oil. $\mathrm{R}_{f}=0.62$ (hexane/EtOAc $=10: 1)$. Note: Products 4r and 4ra could not be separated by silica gel chromatography and were found to be unstable under preparatory TLC conditions. Therefore, the purity is limited and ${ }^{13} \mathrm{C}$ NMR spectra could not be obtained. ${ }^{1} \mathbf{H}$ NMR $(400 \mathrm{MHz}$, Chloroform- $d) \delta 8.46-8.30(\mathrm{~m}, 1 \mathrm{H}), 7.17-7.05(\mathrm{~m}, 1 \mathrm{H}), 6.83(\mathrm{td}, J=10.6$, 9.6, 5.2 Hz, 1H), $6.02(\mathrm{dd}, J=15.9,2.9 \mathrm{~Hz}, 1 \mathrm{H}), 3.80(\mathrm{~d}, J=3.2 \mathrm{~Hz}, 3 \mathrm{H}), 3.16(\mathrm{~s}, 1 \mathrm{H}), 3.05(\mathrm{~s}, 2 \mathrm{H}), 2.40(\mathrm{t}, J=23.9$ $\mathrm{Hz}, 6 \mathrm{H}), 2.06-1.86(\mathrm{~m}, 3 \mathrm{H}), 1.78(\mathrm{~s}, 2 \mathrm{H}), 1.73-1.59(\mathrm{~m}, 2 \mathrm{H}), 1.47-1.29(\mathrm{~m}, 4 \mathrm{H}) .{ }^{19} \mathbf{F}$ NMR (376 MHz, Chloroformd) $\delta$-105.84. IR $\left(\mathrm{KBr}, \mathrm{cm}^{-1}\right)$ 2950, 2869, 1721, 1631, 1257, 1164, 1071, 814. HRMS (ESI) m/z: $[\mathrm{M}+\mathrm{H}]^{+} \mathrm{Calcd}$ for $\mathrm{C}_{24} \mathrm{H}_{29} \mathrm{FO}_{2} \mathrm{H}$ : 369.2230; Found: 369.2228. 


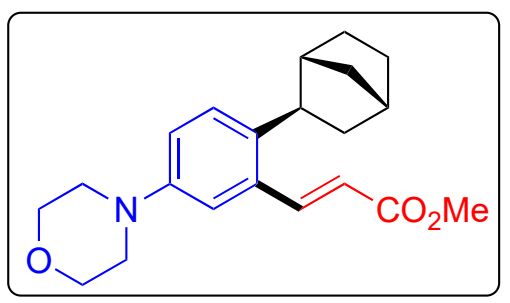

methyl (E)-3-(2-(bicyclo[2.2.1]heptan-2-yl)-5-morpholinophenyl)acrylate (4s): Synthesized from 1s, 2a, and 3a according to the general procedure. $0.2 \mathrm{mmol}$ scale: $32 \%$ yield $(22.1 \mathrm{mg}, 9: 1 \mathrm{E:Z})$. Yellow oil. $\mathrm{R}_{f}=0.36$ (hexane/EtOAc $=4: 1)$. Note: data shown for $Z$ isomer. ${ }^{1} \mathbf{H}$ NMR $(400 \mathrm{MHz}$, Chloroform- $d) \delta 7.23-7.15(\mathrm{~m}, 2 \mathrm{H})$, $6.90(\mathrm{~d}, J=2.7 \mathrm{~Hz}, 1 \mathrm{H}), 6.83(\mathrm{dd}, J=8.6,2.8 \mathrm{~Hz}, 1 \mathrm{H}), 6.00(\mathrm{~d}, J=12.2 \mathrm{~Hz}, 1 \mathrm{H}), 3.88-3.81(\mathrm{~m}, 4 \mathrm{H}), 3.62(\mathrm{~s}, 3 \mathrm{H})$, $3.16-3.12(\mathrm{~m}, 1 \mathrm{H}), 3.15-3.07(\mathrm{~m}, 4 \mathrm{H}), 2.69(\mathrm{dd}, J=9.1,5.5 \mathrm{~Hz}, 1 \mathrm{H}), 2.37-2.29(\mathrm{~m}, 2 \mathrm{H}), 1.69(\mathrm{ddd}, J=11.6,8.9$, $2.3 \mathrm{~Hz}, 1 \mathrm{H}), 1.61-1.56(\mathrm{~m}, 2 \mathrm{H}), 1.54-1.49(\mathrm{~m}, 2 \mathrm{H}), 1.35-1.26(\mathrm{~m}, 3 \mathrm{H}) .{ }^{13} \mathbf{C} \mathbf{~ N M R}\left(101 \mathrm{MHz}, \mathrm{CDCl}_{3}\right) \delta 167.7$, 148.3, 143.9, 137.4, 134.3, 132.7, 127.4, 125.8, 119.6, 51.8, 46.3, 41.7, 40.7, 38.9, 36.7, 32.9, 28.7, 22.3. Mixture: IR $\left(\mathrm{KBr}, \mathrm{cm}^{-1}\right)$ 2952, 2868, 1729, 1606, 1450, 1236, 1122, 818. HRMS (ESI) m/z: [M+Na] ${ }^{+}$Calcd for $\mathrm{C}_{21} \mathrm{H}_{27} \mathrm{NO}_{3} \mathrm{Na}$ : 364.1889; Found: 364.1884 .

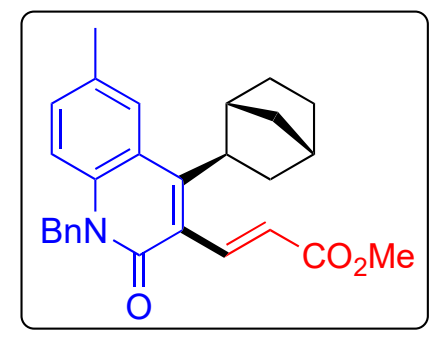

methyl (E)-3-(1-benzyl-4-(bicyclo[2.2.1]heptan-2-yl)-6-methyl-2-oxo-1,2-dihydroquinolin-3-yl)acrylate (4t): Synthesized from 1t, 2a, and 3a according to the general procedure. $0.2 \mathrm{mmol}$ scale: $59 \%$ yield $(50.0 \mathrm{mg})$. Yellow solid. Melting point: $74-77^{\circ} \mathrm{C} . \mathrm{R}_{f}=0.2$ (hexane/EtOAc $\left.=4: 1\right) .{ }^{1} \mathbf{H}$ NMR $(400 \mathrm{MHz}$, Chloroform- $d) \delta 8.16(\mathrm{~d}, J=$ $16.0 \mathrm{~Hz}, 1 \mathrm{H}), 7.79(\mathrm{~s}, 1 \mathrm{H}), 7.32-7.27(\mathrm{~m}, 1 \mathrm{H}), 7.25-7.12(\mathrm{~m}, 6 \mathrm{H}), 6.81(\mathrm{~d}, J=15.9 \mathrm{~Hz}, 1 \mathrm{H}), 5.54(\mathrm{br} \mathrm{s}, 2 \mathrm{H}), 3.80$ (s, 3H), 3.35 (dd, $J=9.5,7.6 \mathrm{~Hz}, 1 \mathrm{H}), 2.87(\mathrm{~s}, 1 \mathrm{H}), 2.42(\mathrm{~s}, 1 \mathrm{H}), 2.40(\mathrm{~s}, 3 \mathrm{H}), 2.04$ (t, $J=10.8 \mathrm{~Hz}, 1 \mathrm{H}), 1.88(\mathrm{~d}, J=$ $9.9 \mathrm{~Hz}, 1 \mathrm{H}), 1.72(\mathrm{~d}, J=9.5 \mathrm{~Hz}, 3 \mathrm{H}), 1.52(\mathrm{~d}, J=10.6 \mathrm{~Hz}, 1 \mathrm{H}), 1.44(\mathrm{~d}, J=2.4 \mathrm{~Hz}, 1 \mathrm{H}), 1.42(\mathrm{~s}, 1 \mathrm{H}) .{ }^{13} \mathbf{C} \mathbf{~ N M R}(101$ $\left.\mathrm{MHz}, \mathrm{CDCl}_{3}\right) \delta 167.7,148.3,143.9,137.4,134.3,132.7,127.4,125.8,119.6,51.8,46.3,41.7,40.7,38.9,36.7,32.9$, 28.7, 22.3. IR (KBr, cm $\left.{ }^{-1}\right)$ 2951, 2870, 1719, 1647, 1497, 1437, 1299, 1271, 1169, 807, 728. HRMS (ESI) m/z: $[\mathrm{M}+\mathrm{Na}]^{+}$Calcd for $\mathrm{C}_{28} \mathrm{H}_{29} \mathrm{NO}_{3} \mathrm{Na}$ : 450.2045; Found: 450.2043 .

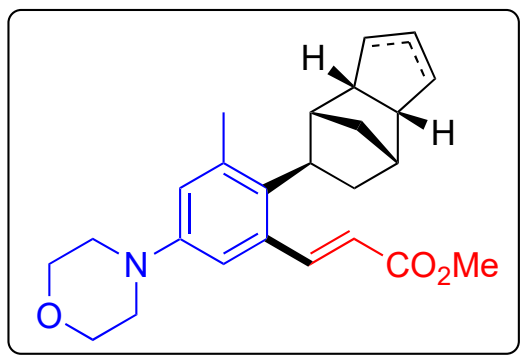

Methyl (E)-3-(2-(3a,4,5,6,7,7a-hexahydro-1 H-4,7-methanoinden-5/6-yl)-3-methyl-5-morph-olinophenyl)acrylate (4u): Synthesized from 1a, $2 \mathbf{a}$, and $\mathbf{3 u}$ according to the general procedure. $0.2 \mathrm{mmol}$ scale: $77 \%$ yield $(60.3 \mathrm{mg}$, $r . r .=1: 1)$. Yellow oil. $\mathrm{R}_{f}=0.37$ (hexane/EtOAc $\left.=4: 1\right) .{ }^{1} \mathbf{H}$ NMR $(400 \mathrm{MHz}$, Chloroform- $d) \delta 8.40(\mathrm{~m}, 1 \mathrm{H}), 6.75(\mathrm{~m}$, $1 \mathrm{H}), 6.69(\mathrm{~m}, 1 \mathrm{H}), 6.08(\mathrm{~m}, 1 \mathrm{H}), 5.85-5.74(\mathrm{~m}, 1 \mathrm{H}), 5.74-5.62(\mathrm{~m}, 1 \mathrm{H}), 3.87-3.82(\mathrm{~m}, 4 \mathrm{H}), 3.81(\mathrm{~s}, 3 \mathrm{H}), 3.25-$ $3.17(\mathrm{~m}, 2 \mathrm{H}), 3.14-3.07(\mathrm{~m}, 4 \mathrm{H}), 3.09-3.04(\mathrm{~m}, 1 \mathrm{H}), 2.99(\mathrm{t}, J=8.6 \mathrm{~Hz}, 0.5 \mathrm{H}), 2.63(\mathrm{~m}, 1 \mathrm{H}), 2.53(\mathrm{~m}, 1 \mathrm{H}), 2.44-$ $2.35(\mathrm{~m}, 1 \mathrm{H}), 2.33(\mathrm{~m}, 3 \mathrm{H}), 2.30-2.22(\mathrm{~m}, 2 \mathrm{H}), 2.00(\mathrm{~m}, 1 \mathrm{H}), 1.93-1.83(\mathrm{~m}, 1 \mathrm{H}), 1.64(\mathrm{~s}, 0.5 \mathrm{H}), 1.61(\mathrm{~s}, 0.5 \mathrm{H})$, $1.56(\mathrm{q}, J=3.0,2.3 \mathrm{~Hz}, 0.5 \mathrm{H}) .{ }^{13} \mathbf{C}$ NMR $\left(101 \mathrm{MHz}, \mathrm{CDCl}_{3}\right) \delta 167.7,148.3,143.9,137.4,134.3,132.7,127.4,125.8$, 119.6, 51.8, 46.3, 41.7, 40.7, 38.9, 36.7, 32.9, 28.7, 22.3. IR (KBr, $\left.\mathrm{cm}^{-1}\right)$ 2949, 2851, 1718, 1597, 1168, 1122. HRMS (ESI) $\mathrm{m} / \mathrm{z}:[\mathrm{M}+\mathrm{Na}]^{+}$Calcd for $\mathrm{C}_{25} \mathrm{H}_{31} \mathrm{NO}_{3} \mathrm{Na}$ : 416.2202; Found: 416.2201 . 


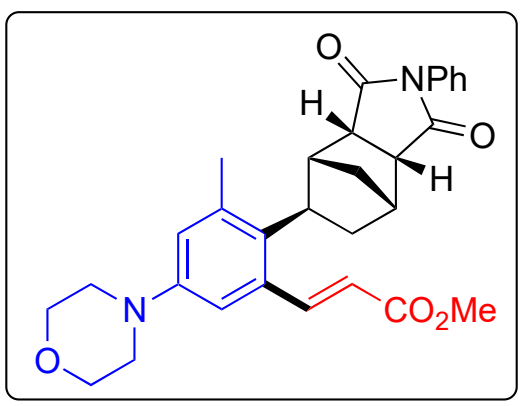

methyl

(E)-3-(2-endo-(1,3-dioxo-2-phenyloctahydro-1 H-4,7-methanoisoindol-5-yl)-3-methyl-5morpholinophenyl)acrylate (4v): Synthesized from 1t, 2a, and 3a according to the general procedure. 0.2 mmol scale: $68 \%$ yield $(67.8 \mathrm{mg})$. Orange solid. Melting point: $112-115{ }^{\circ} \mathrm{C} . \mathrm{R}_{f}=0.11$ (hexane/EtOAc $\left.=4: 1\right) .{ }^{1} \mathbf{H}$ NMR $(400 \mathrm{MHz}$, Chloroform- $d) \delta 8.30(\mathrm{~d}, J=15.4 \mathrm{~Hz}, 1 \mathrm{H}), 7.53-7.46(\mathrm{~m}, 2 \mathrm{H}), 7.45-7.38(\mathrm{~m}, 1 \mathrm{H}), 7.34-7.29(\mathrm{~m}, 2 \mathrm{H})$, $6.75(\mathrm{~d}, J=2.8 \mathrm{~Hz}, 1 \mathrm{H}), 6.63(\mathrm{~d}, J=2.8 \mathrm{~Hz}, 1 \mathrm{H}), 6.08(\mathrm{~d}, J=15.5 \mathrm{~Hz}, 1 \mathrm{H}), 3.88-3.81(\mathrm{~m}, 4 \mathrm{H}), 3.79(\mathrm{~s}, 3 \mathrm{H}), 3.43-$ $3.30(\mathrm{~m}, 2 \mathrm{H}), 3.23(\mathrm{dd}, J=16.3,6.5 \mathrm{~Hz}, 2 \mathrm{H}), 3.15-3.09(\mathrm{~m}, 4 \mathrm{H}), 2.99(\mathrm{~s}, 1 \mathrm{H}), 2.31(\mathrm{~s}, 3 \mathrm{H}), 2.22-2.09(\mathrm{~m}, 2 \mathrm{H})$, $1.94-1.80(\mathrm{~m}, 2 \mathrm{H}) .{ }^{13} \mathbf{C}$ NMR $\left(101 \mathrm{MHz} \mathrm{CDCl}_{3}\right) \delta 177.2,177.1,167.2,149.1,148.5,138.7,134.9,132.8,132.0$, 129.4, 128.9, 126.7, 120.9, 120.2, 114.8, 51.9, 49.8, 49.1, 48.8, 45.1, 42.2, 41.1, 39.7, 35.4, 22.9. IR (KBr, $\left.\mathrm{cm}^{-1}\right)$ 2957, 2852, 1711, 1628, 1598, 1251, 1160, 1122. HRMS (ESI) m/z: [M+H] Calcd for $\mathrm{C}_{30} \mathrm{H}_{32} \mathrm{~N}_{2} \mathrm{O}_{5} \mathrm{H}$ : 501.2389; Found: 501.2388. 


\section{X-ray data for $4 d$}

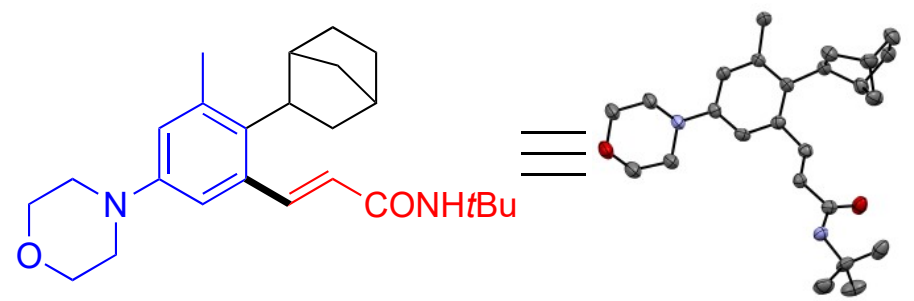

The enantiomer on the norbornyl ring and hydrogen atoms were omitted for clarity; thermal ellipsoids drawn at $50 \%$ probability.

(A single crystal was obtained by vapor diffusion of pentane into a solution of $\mathbf{4 d}$ dissolved in ethyl acetate)

Identification code

Empirical formula

Formula weight

Temperature/K

Crystal system

Space group

$\mathrm{a} / \AA$

$\mathrm{b} / \AA$

$\mathrm{c} / \AA$

$\alpha /^{\circ}$

$\beta /{ }^{\circ}$

$\gamma /{ }^{\circ}$

Volume/Å

$\mathrm{Z}$

$\rho_{\text {calc }} / \mathrm{cm}^{3}$

$\mu / \mathrm{mm}^{-1}$

$\mathrm{F}(000)$

Crystal size $/ \mathrm{mm}^{3}$

Radiation 4d (CCDC\# 1990447)

$\mathrm{C}_{25} \mathrm{H}_{36} \mathrm{~N}_{2} \mathrm{O}_{2}$

396.56

100(2)

hexagonal

P65

13.2389(8)

$13.2389(8)$

$22.8347(14)$

90

90

120

$3466.0(5)$

6

1.140

0.072

1296.0

$0.317 \times 0.192 \times 0.126$

$\operatorname{MoK} \alpha(\lambda=0.71073)$

$2 \Theta$ range for data collection $/{ }^{\circ} 5.036$ to 50.786

Index ranges

$-15 \leq \mathrm{h} \leq 15,-15 \leq \mathrm{k} \leq 15,-27 \leq \mathrm{l} \leq 27$

Reflections collected

79372

Independent reflections

$4236\left[\mathrm{R}_{\text {int }}=0.1095, \mathrm{R}_{\text {sigma }}=0.0492\right]$

Data/restraints/parameters 4236/267/312

Goodness-of-fit on $\mathrm{F}^{2}$

1.034

Final $R$ indexes [I>=2 $\sigma(I)]$

$\mathrm{R}_{1}=0.0510, \mathrm{wR}_{2}=0.1027$

Final $R$ indexes [all data]

$\mathrm{R}_{1}=0.0872, \mathrm{wR}_{2}=0.1169$

Largest diff. peak/hole / e $\AA^{-3} 0.23 /-0.19$

Flack parameter

$-0.8(6)$ 


\section{References}

1. Dong, Z.; Dong, G. J. Am. Chem. Soc. 2013, 135, 18350.

2. Wu, T. Y.-H.; Li, Y.; Cortez, A.; Zou,m Y.; Mishra, P.; Zhang, X.; Skibinski, D.; Singh, M.; Valiante, N. U.S. Patent WO 2009-US35563, February 27, 2009.

3. Lu, Z.; Twieg, R. J.; Huang, S. D. Tetrahedron Lett. 2003, 44, 6289.

4. Yamamoto, Y.; Murayama, T.; Jiang, J.; Yasui, T.; Shibuya, M. Chem. Sci. 2018, 5, 1191.

5. Hell, S. M.; Meyer, C. F.; Laudadio, G.; Misale, A.; Willis, M. C.; Noël, T.; Trabanco, A. A.; Gouverneur, V. J. Am. Chem. Soc. 2020, 142, 720.

6. Chen, Z.; Hu, L.; Zeng, F.; Zhu, R.; Zheng, S.; Yu, Q.; Huang, J. Chem. Commun. 2017, 30, 4258.

7. Lautens, M.; Paquin, J.-F.; Piguel, S. J. Org. Chem. 2002, 67, 3972.

8. Della Ca', N.; Maestri, G.; Malacria, M.; Derat, E.; Catellani, M. Angew. Chem., Int. Ed. 2011, $50,12257$.

9. Dostas, I. D.; Andreadaki, F. J.; Medlycott, E. A.; Hanan, G. S.; Monflier, E. Tetrahedron Lett. $2009,50,1851$.

10. Mo, F.; Yan, J. M.; Qiu, D.; Li, F.; Zhang, Y.; Wang, J. Angew. Chem., Int. Ed. 2010, 49, 2028.

11. Bechtoldt, A.; Ackermann, L. ChemCatChem 2019, 11, 435.

12. Bonvallet, P. A.; Breitkreuz, C. J.; Kim, Y. S.; Todd, E. M.; Traynor, K.; Fry, C. G.; Ediger, M. D.; McMahon, R. J. J. Org. Chem. 2007, 72, 10051.

13. Catellani, M.; Cugini, F. Tetrahedron 1999, 55, 6595. 
8. ${ }^{1} \mathrm{H},{ }^{2} \mathrm{H},{ }^{13} \mathrm{C}$, and ${ }^{19} \mathrm{~F}$ NMR Spectra
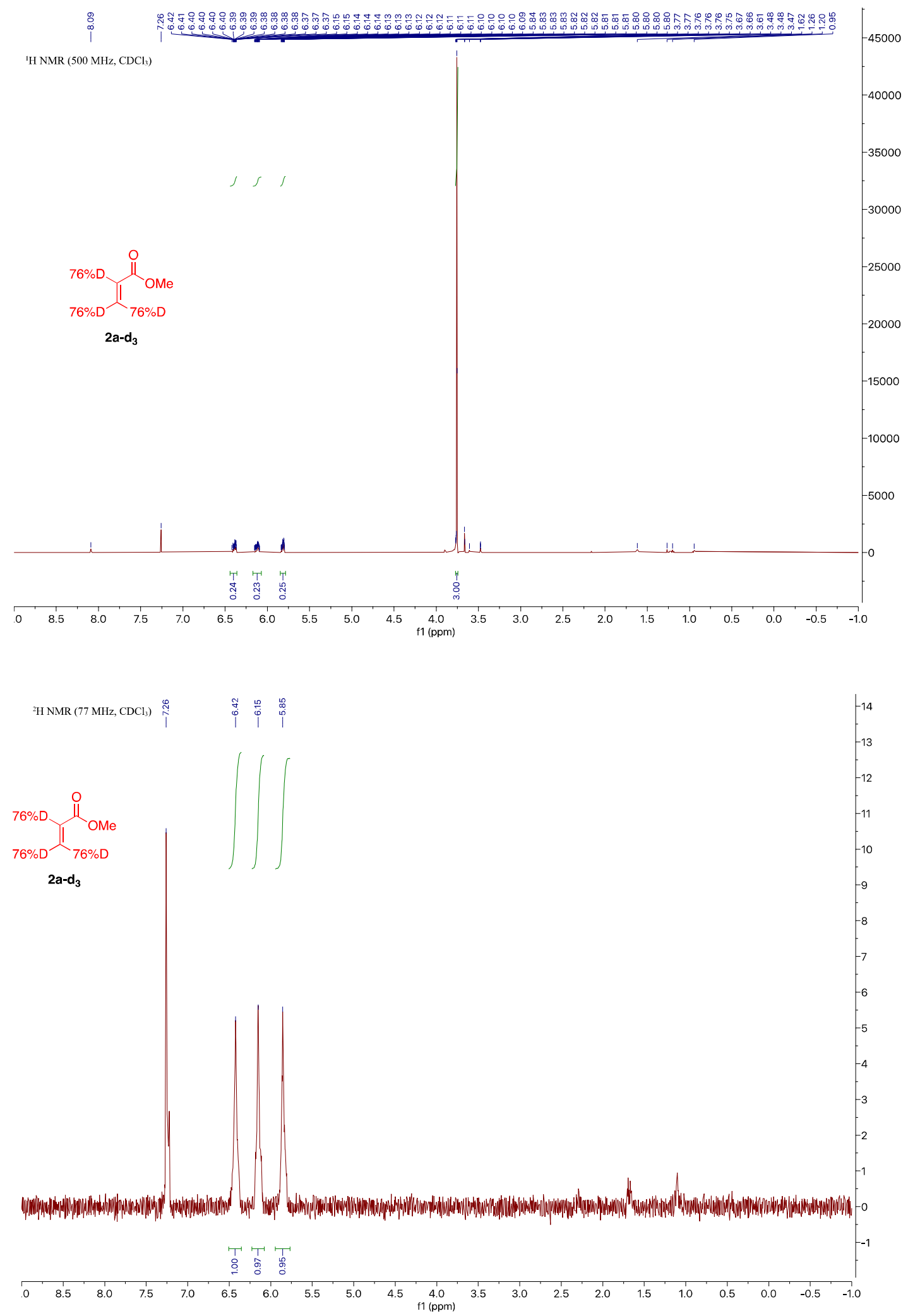


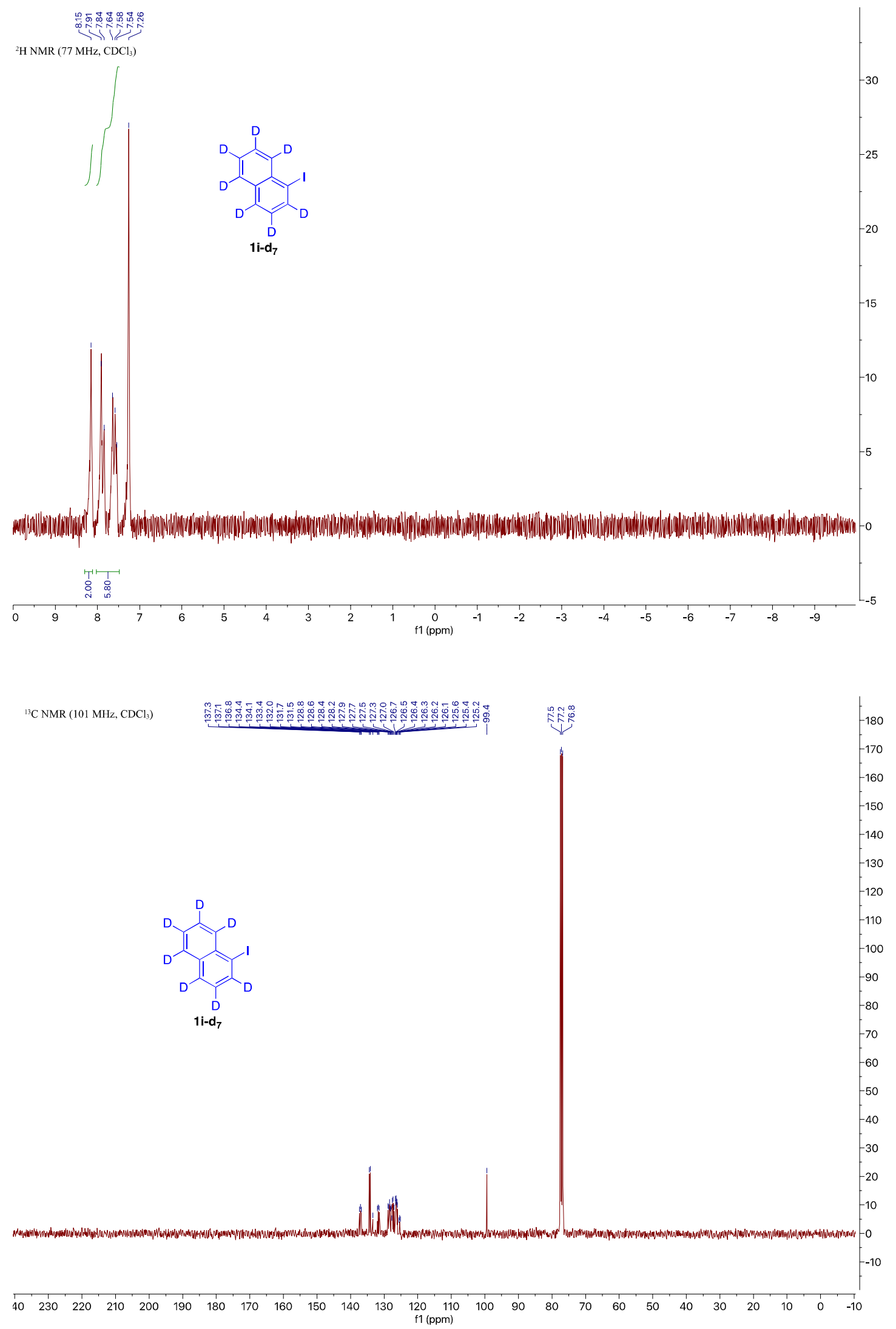




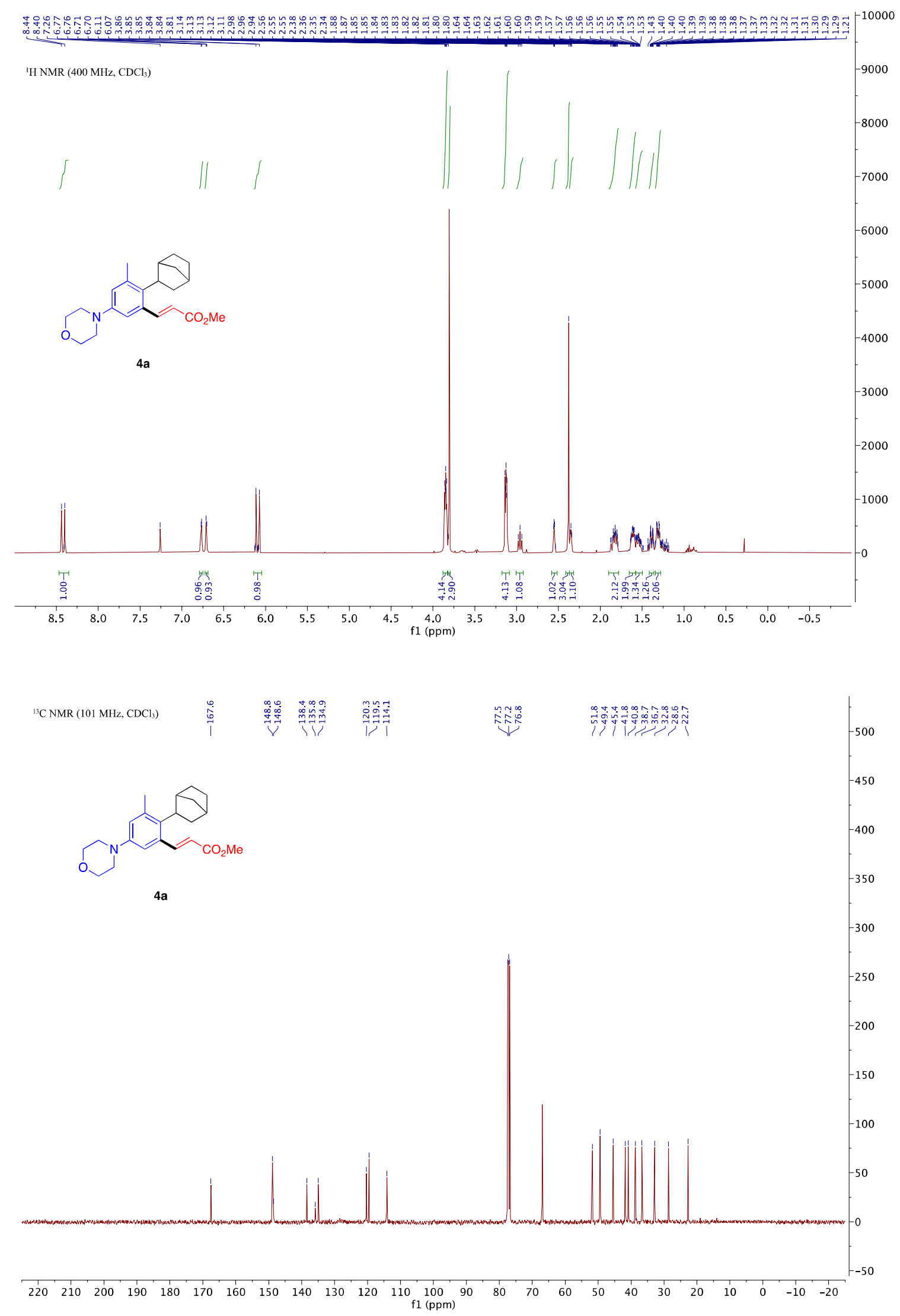



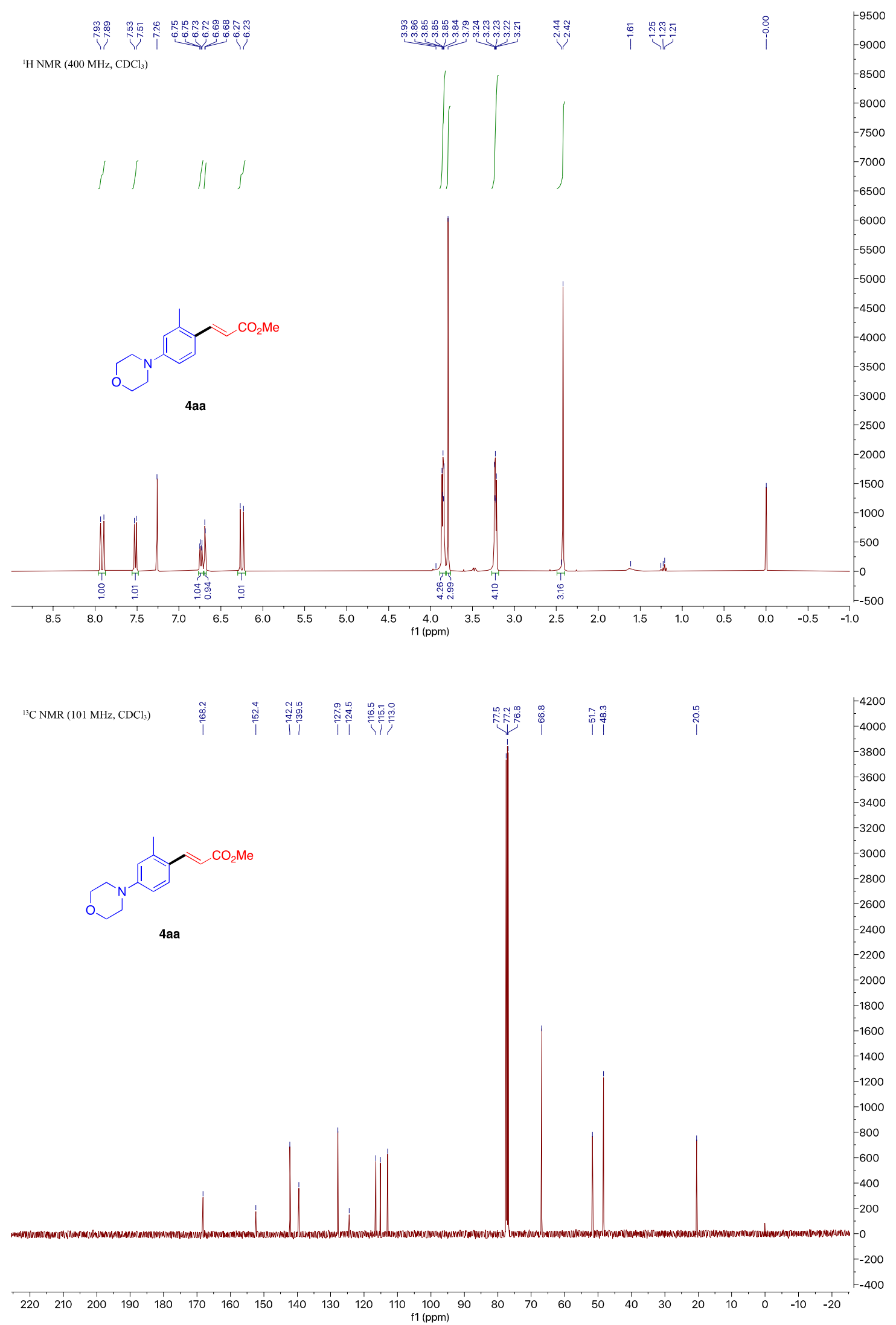


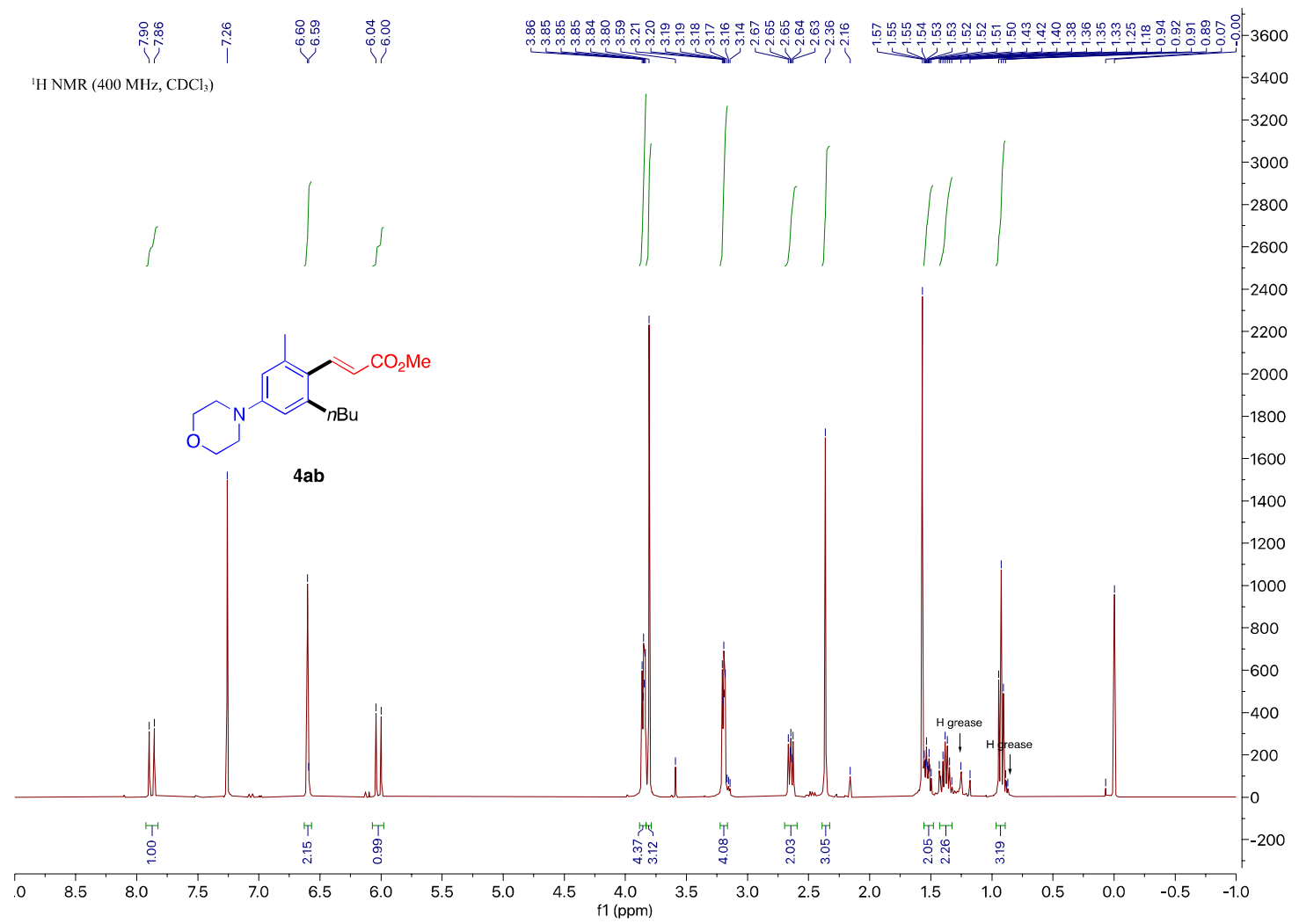

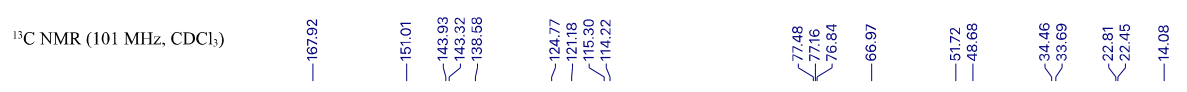
4000

500<smiles>CCCCc1cc(N2CCOCC2)cc(C)c1/C=C/C(=O)OC</smiles>

$4 a b$

$-2500$

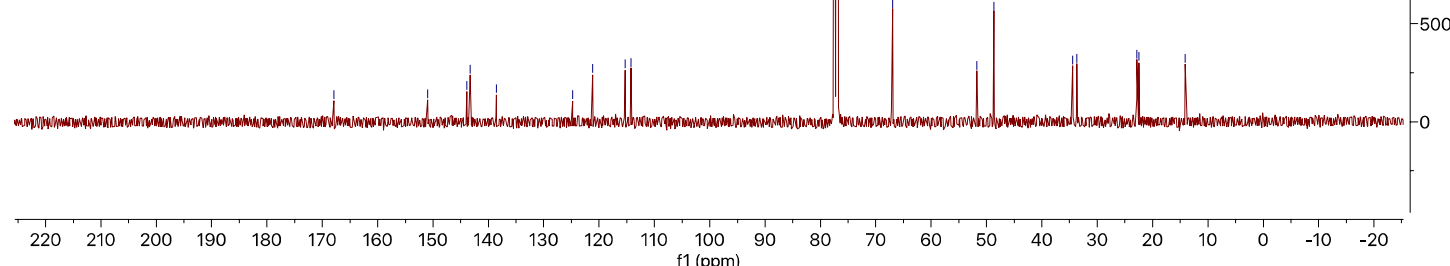



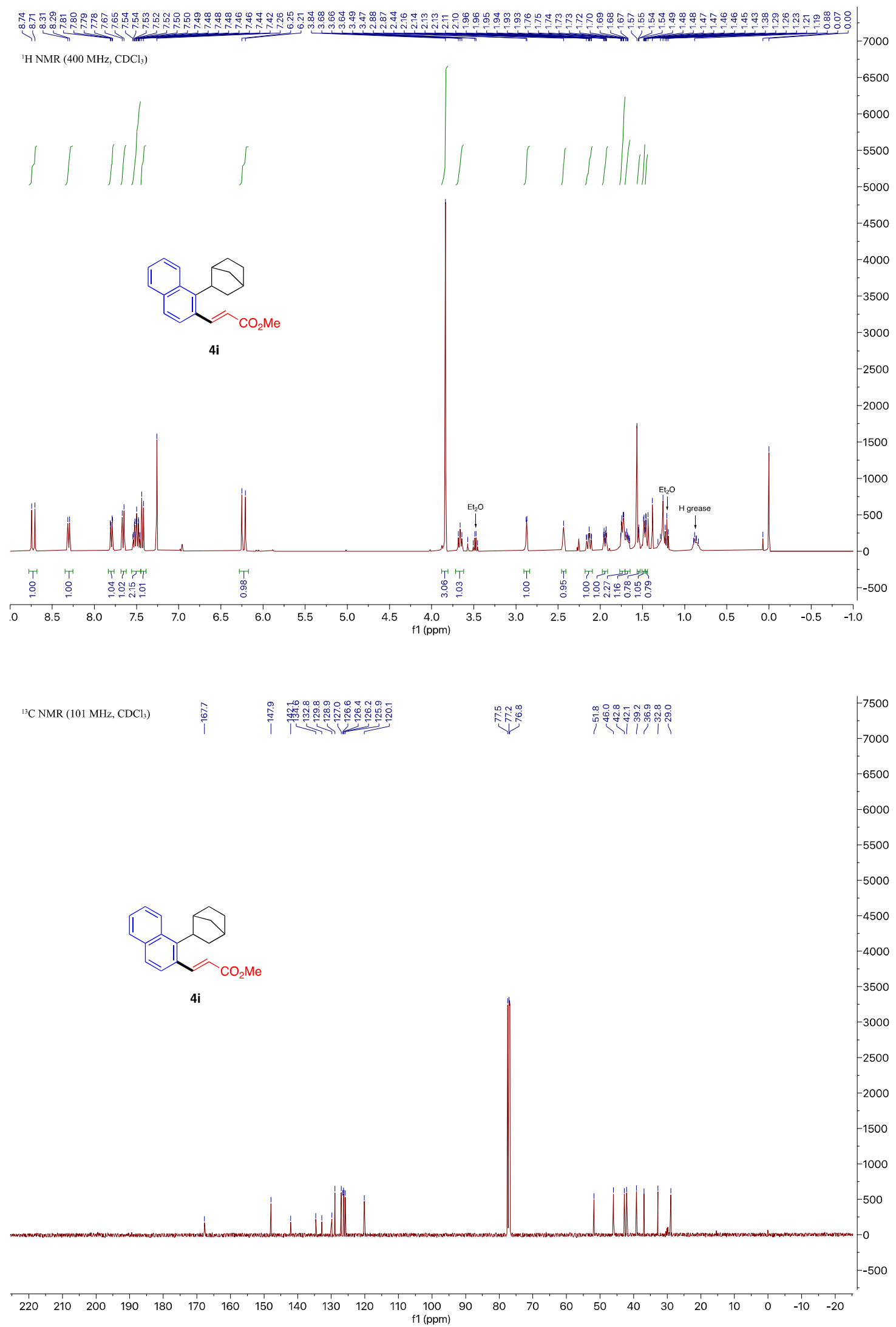


\section{HSQC Spectrum of $4 i$}

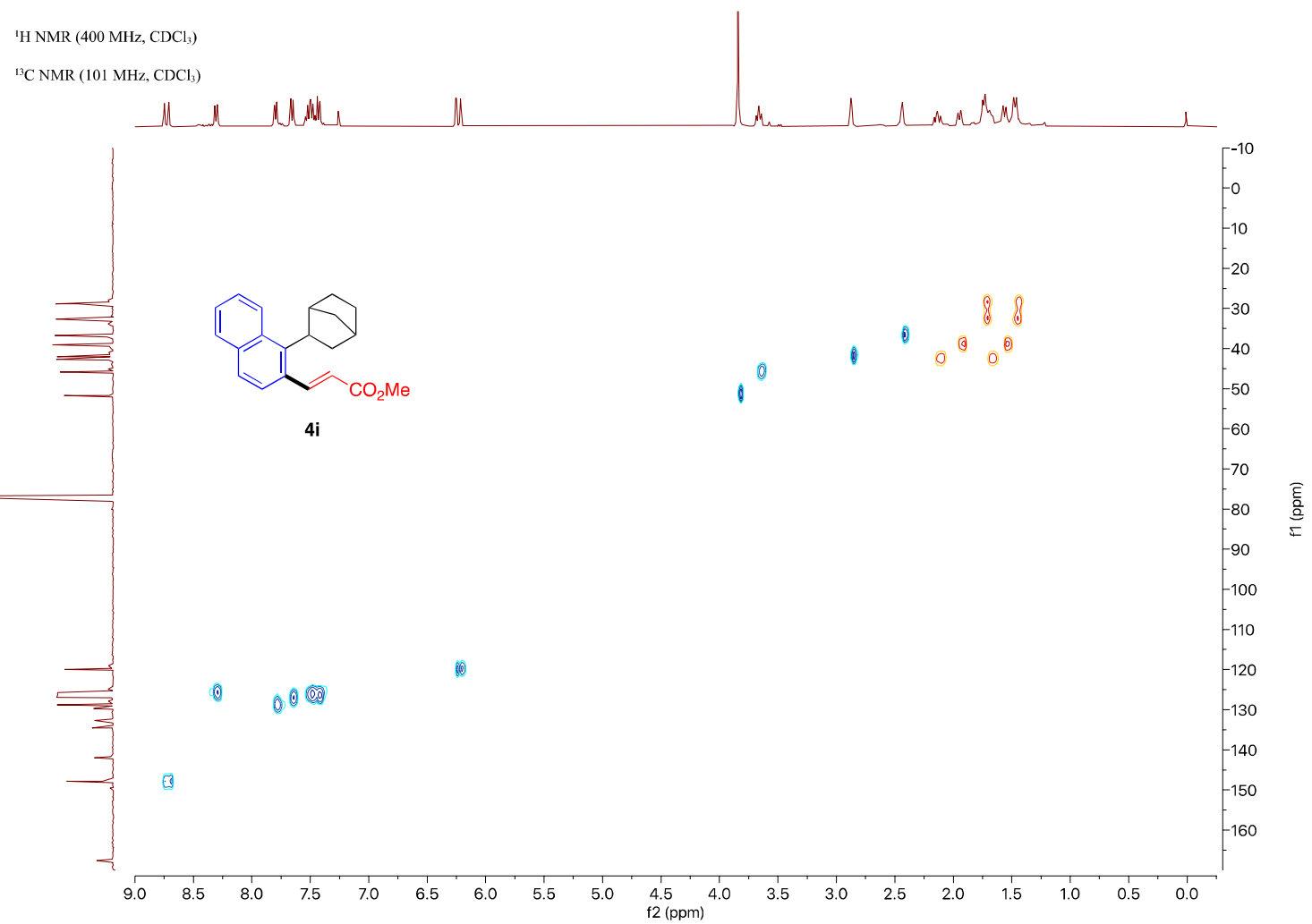

\section{COSY Spectrum of $4 i$}

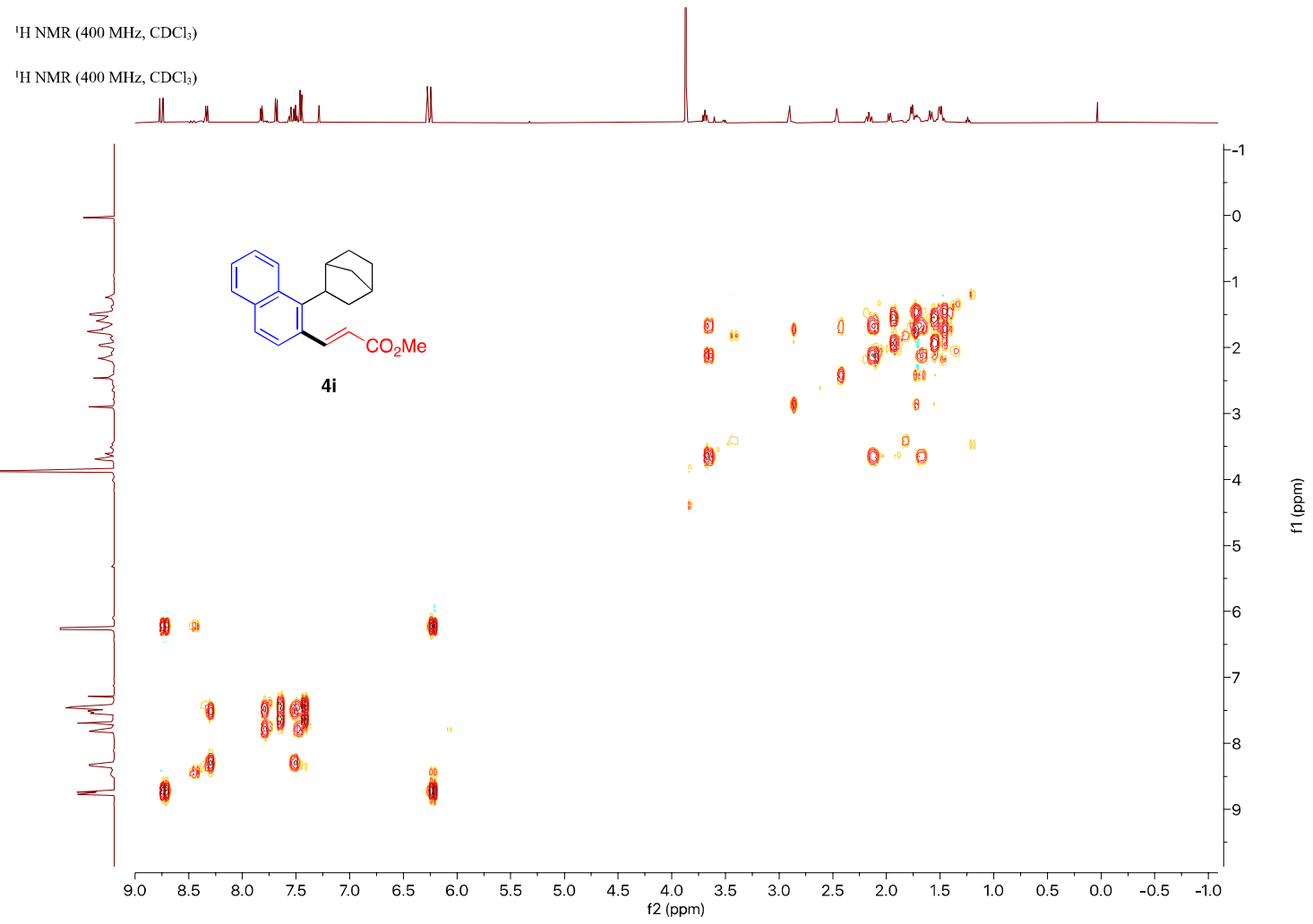




\section{NOSEY Spectrum of $4 \mathrm{i}$ (alkyl region)}

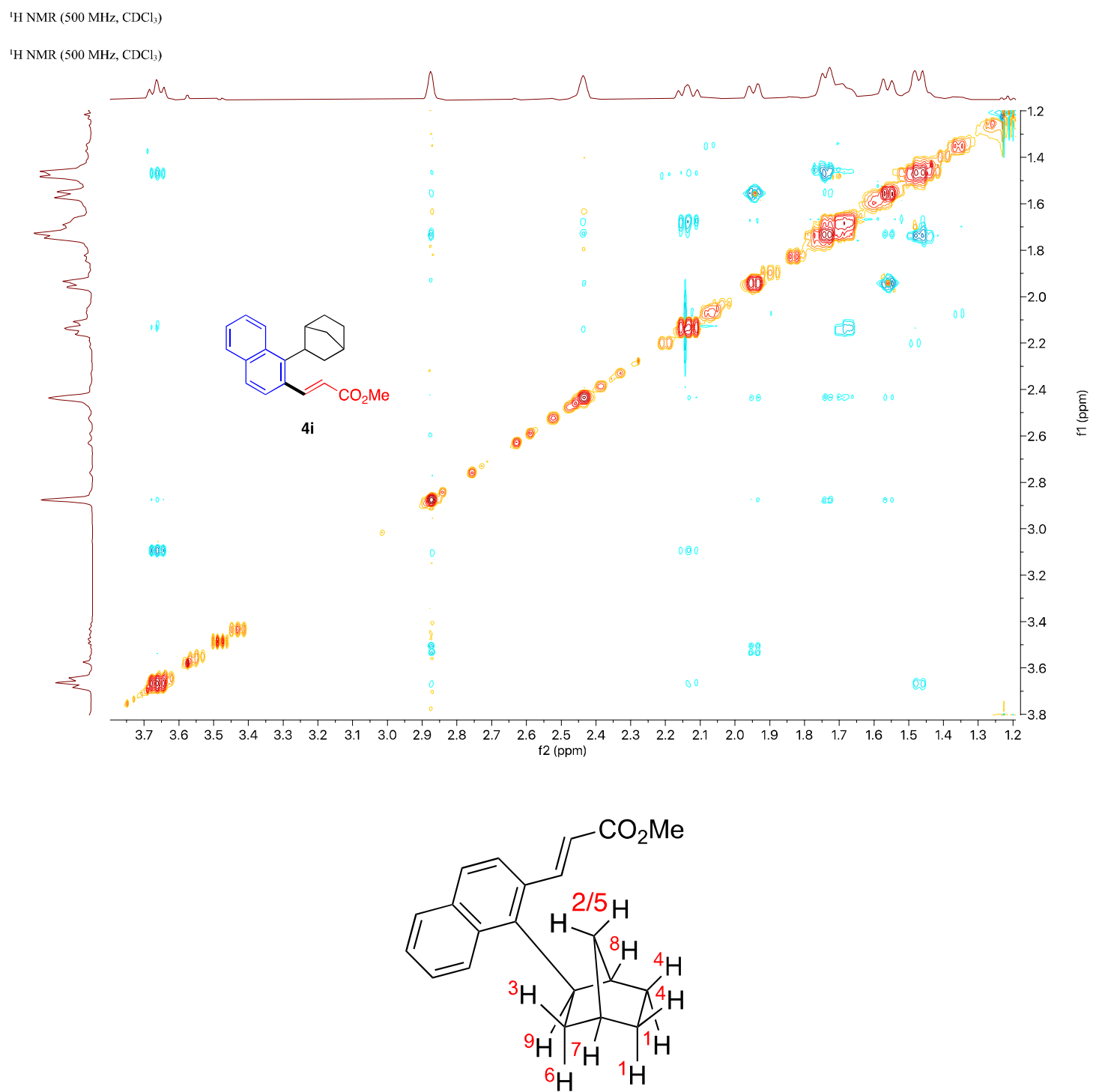



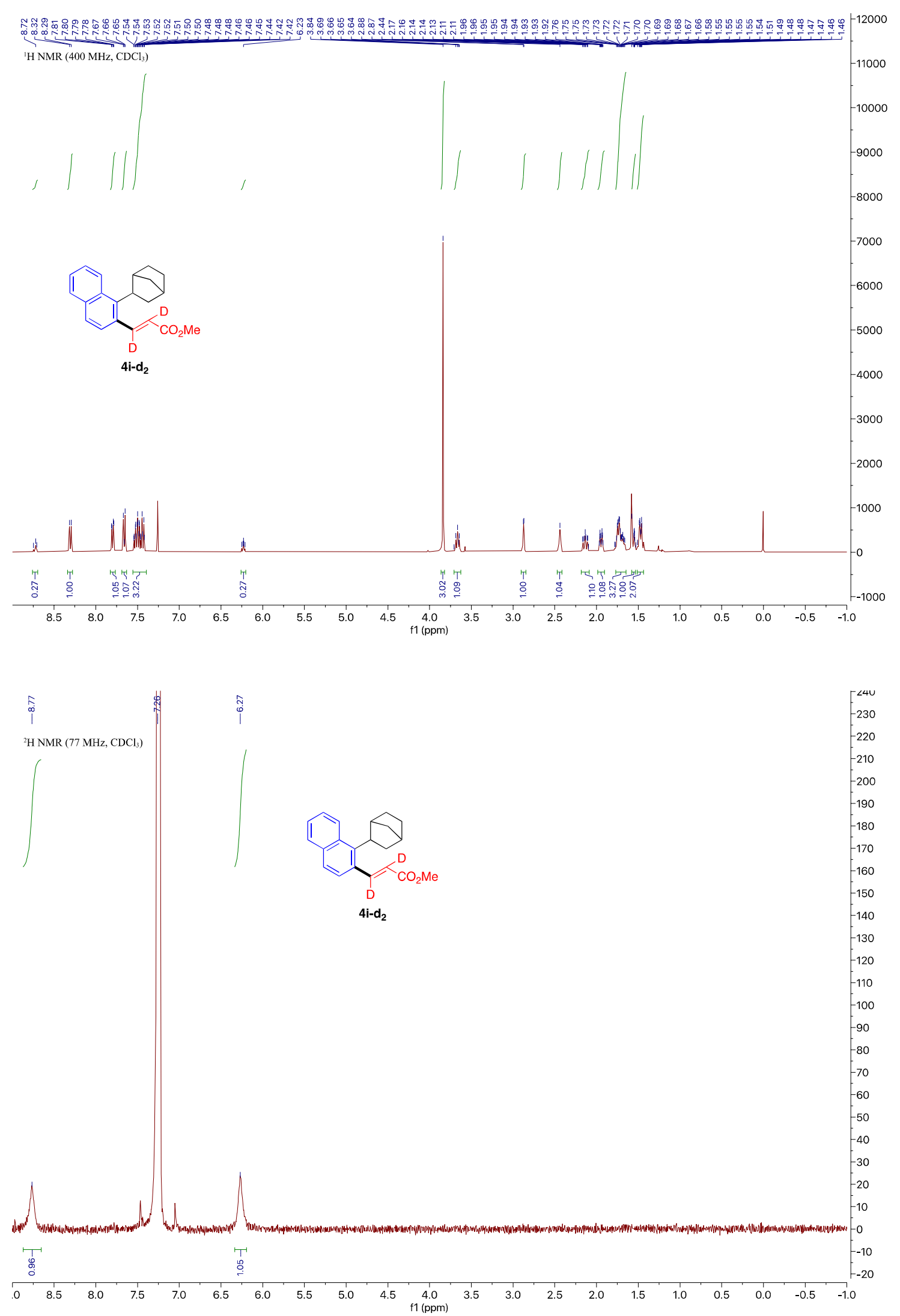


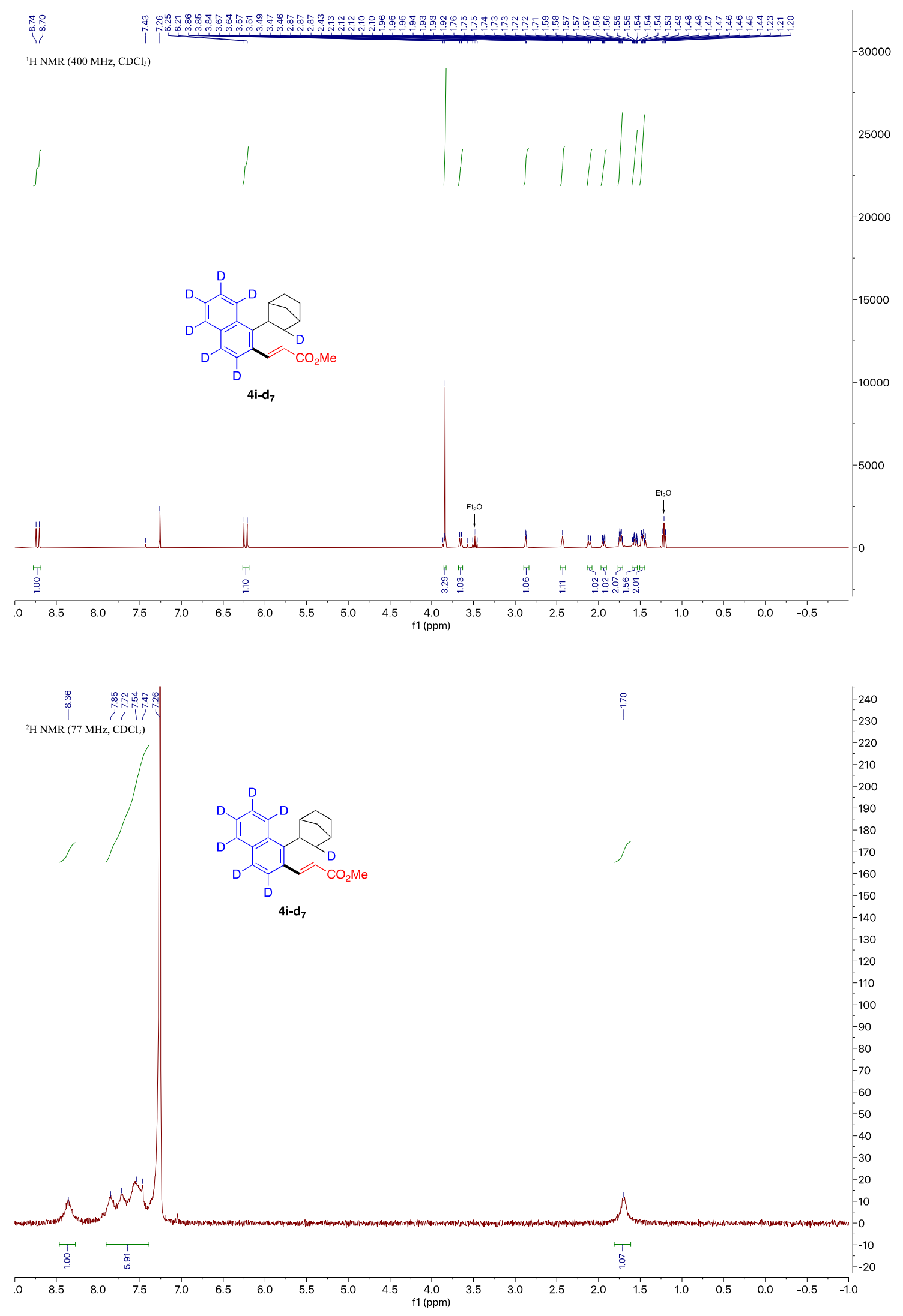



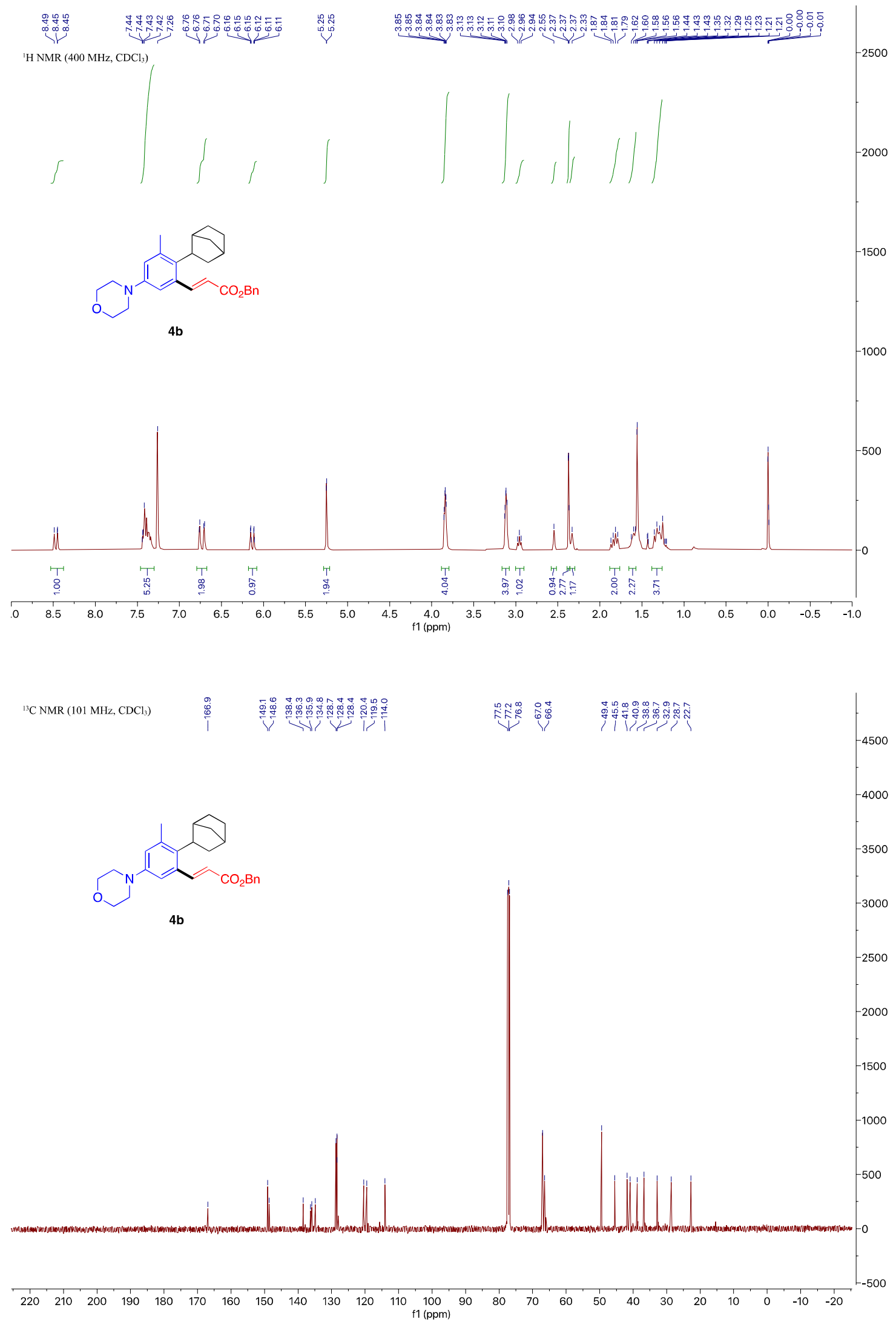


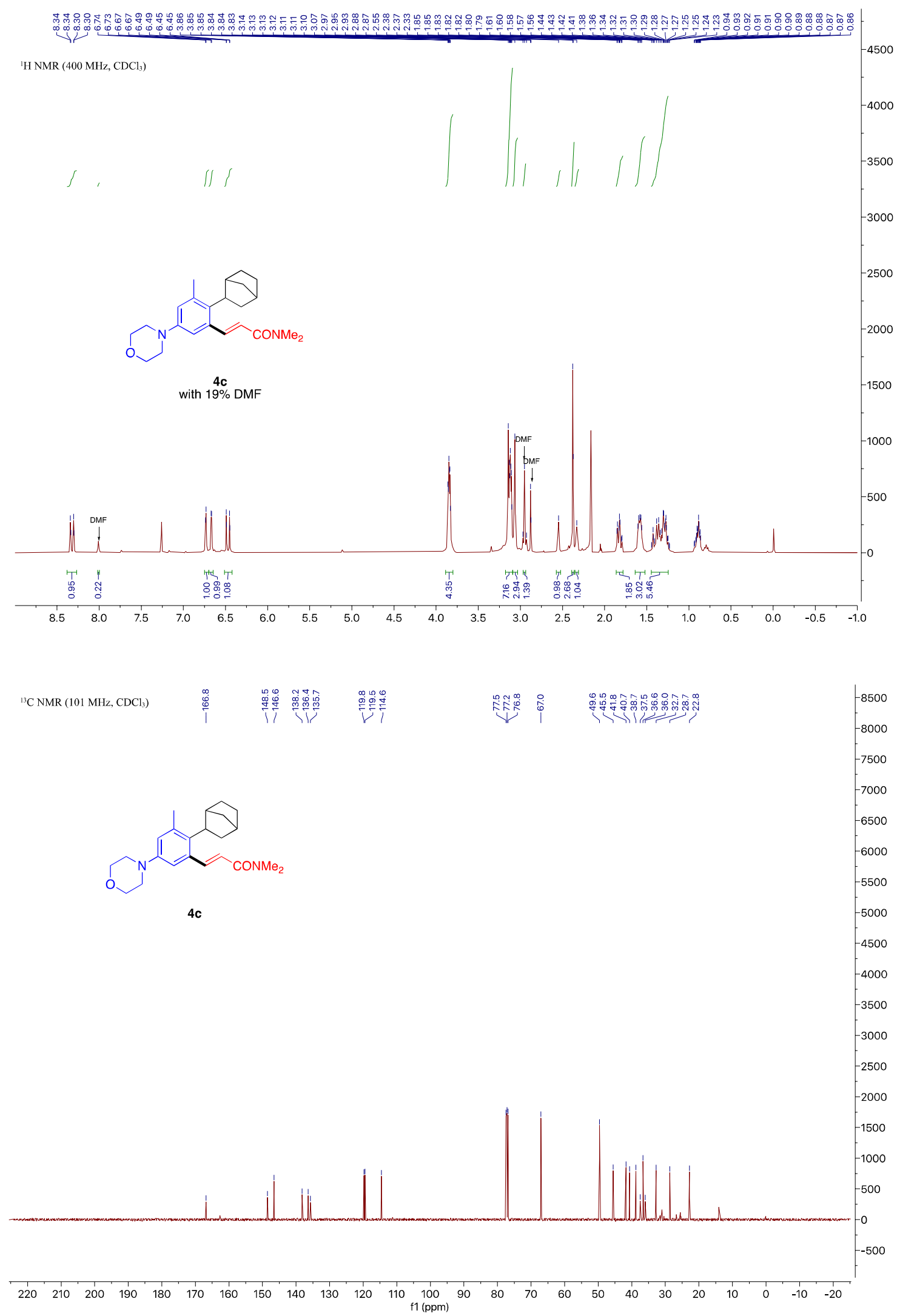




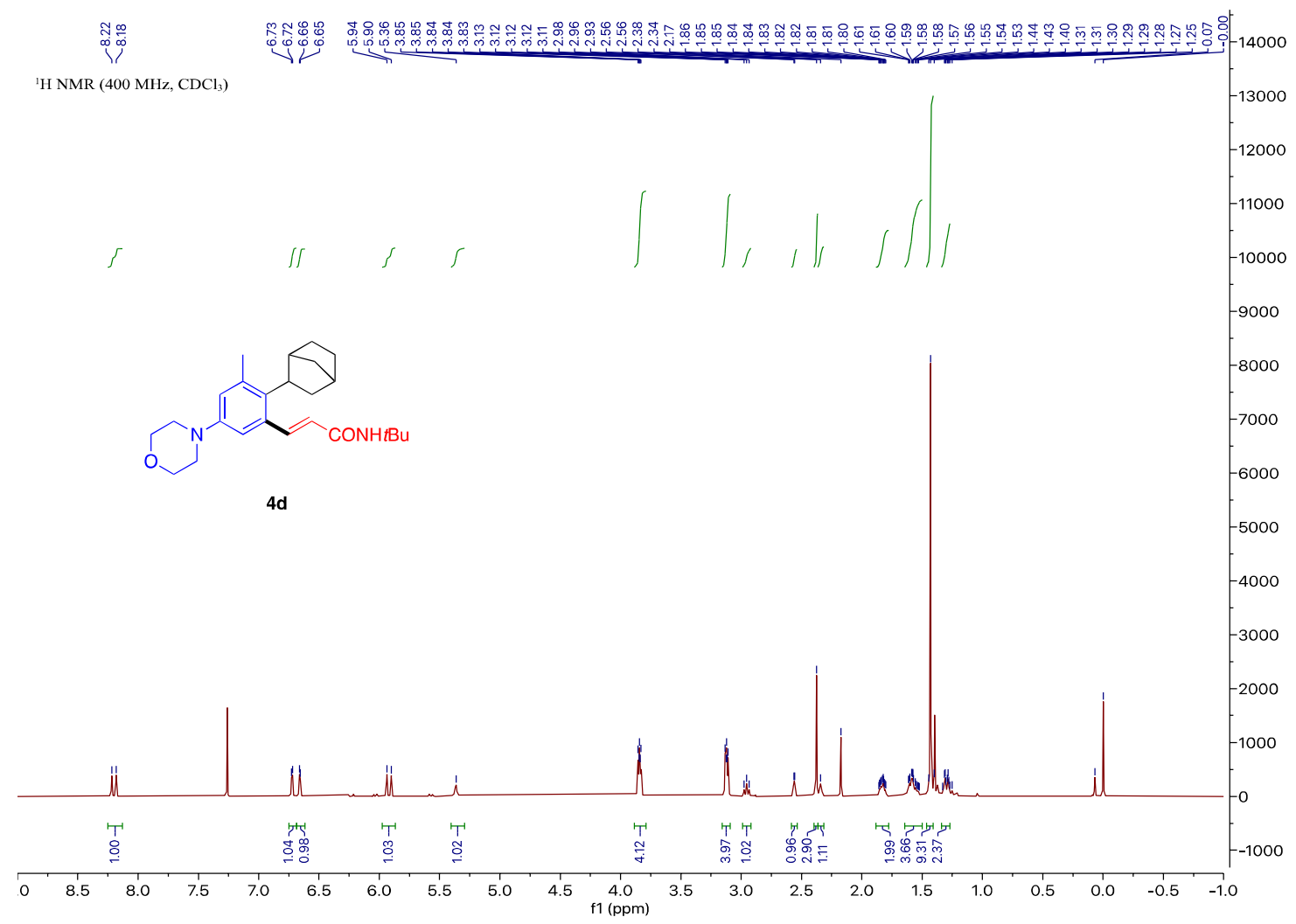

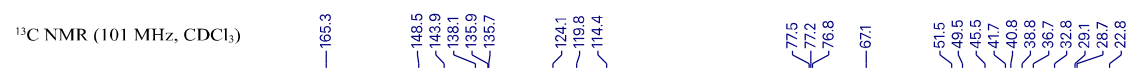

$-2300$

$-2200$

$-2100$

$-2000$

1900<smiles>CCCCNC(=O)C=Cc1cc(N2CCOCC2)cc(C)c1C1CC2CCC1C2</smiles>

4d

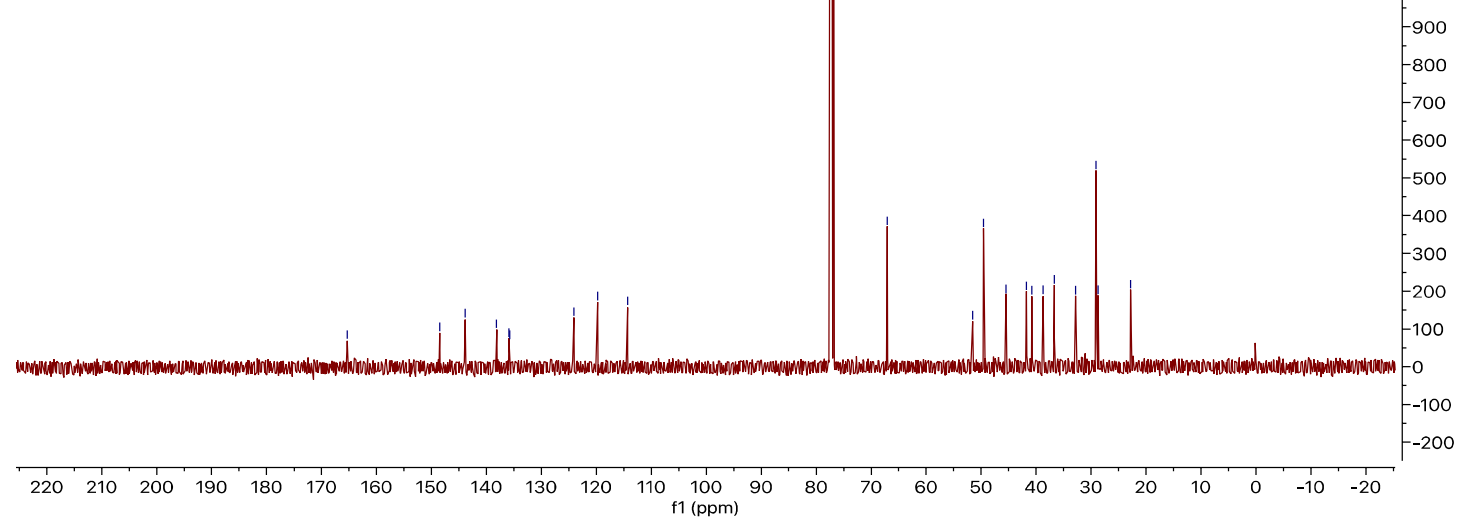




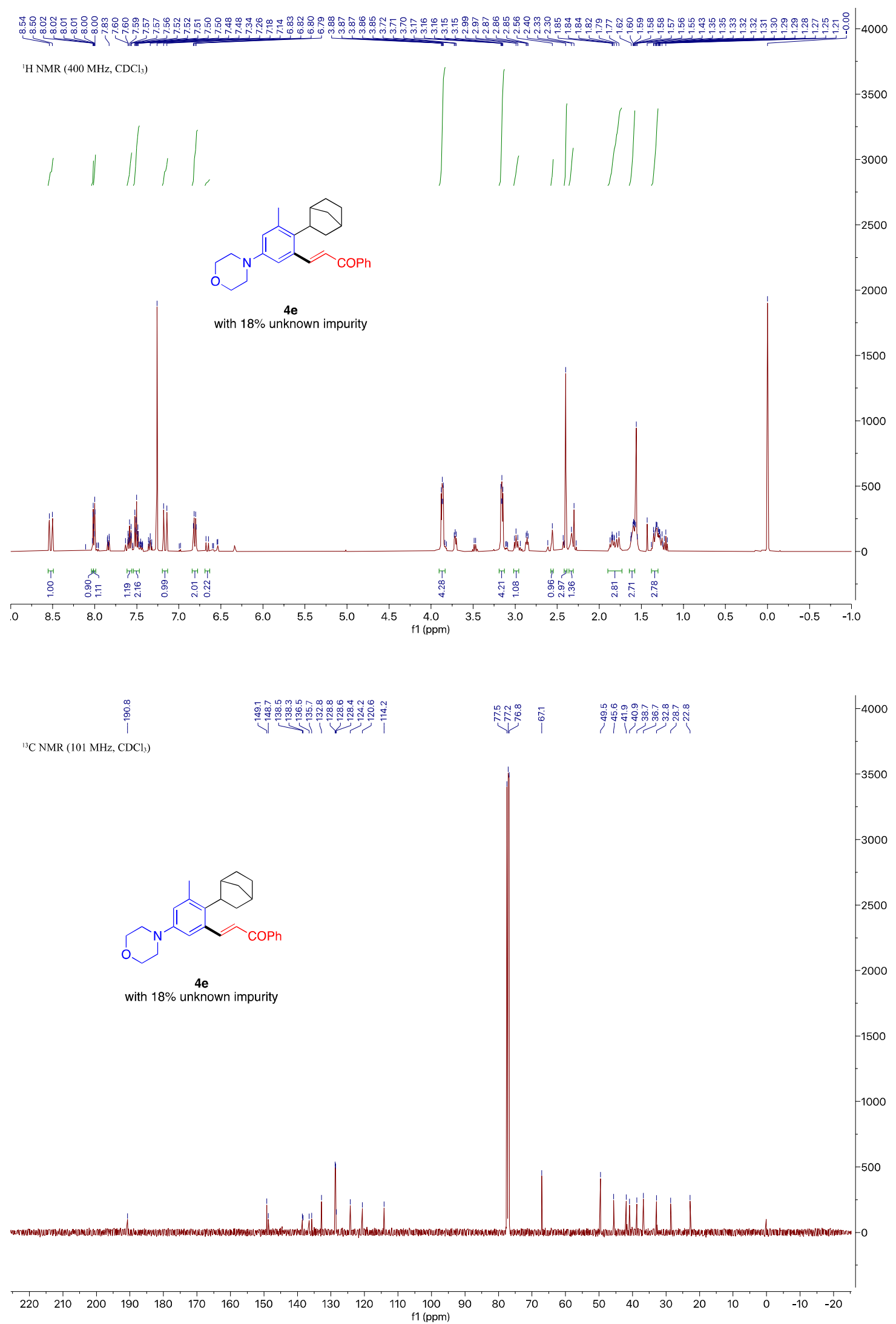



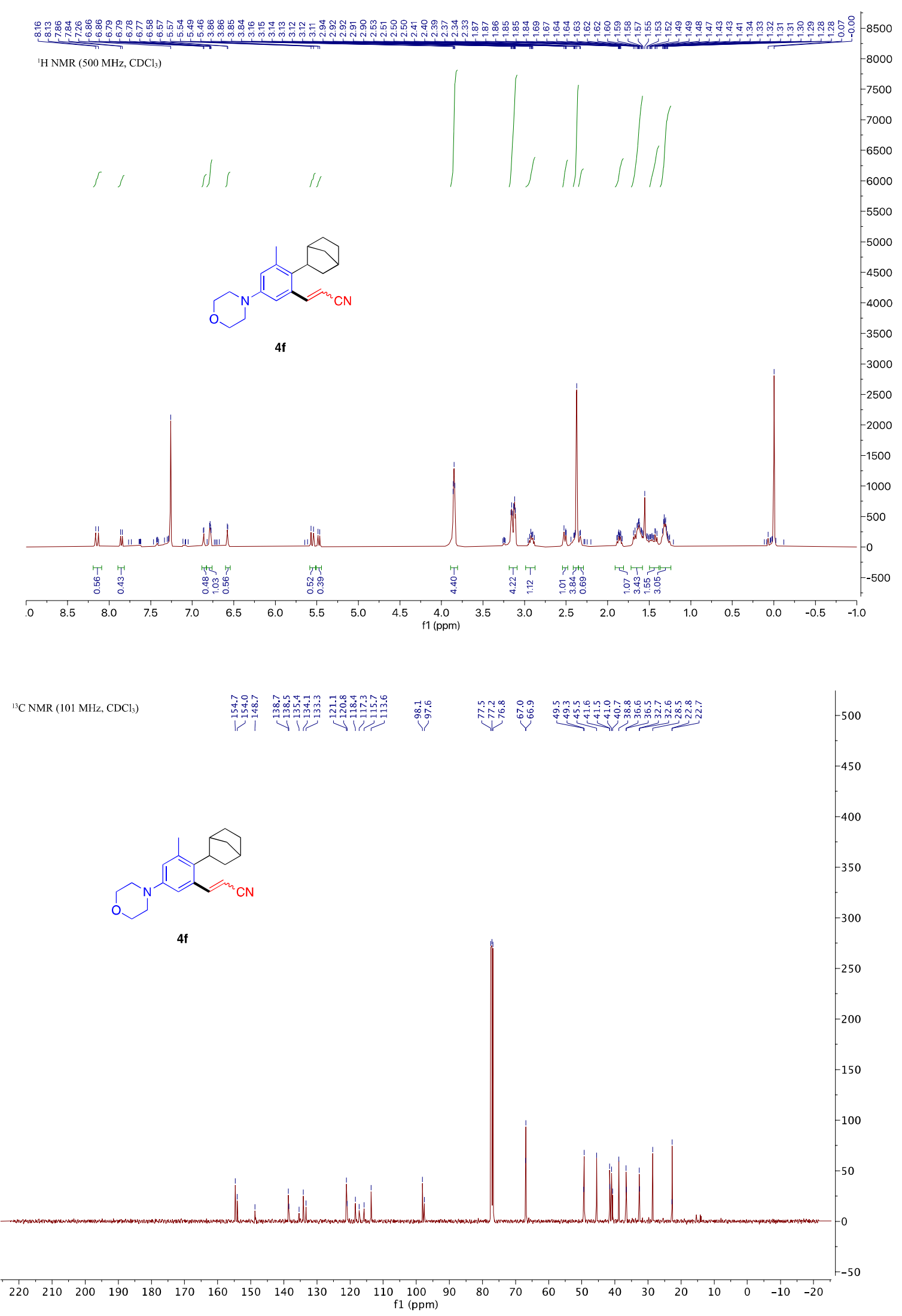

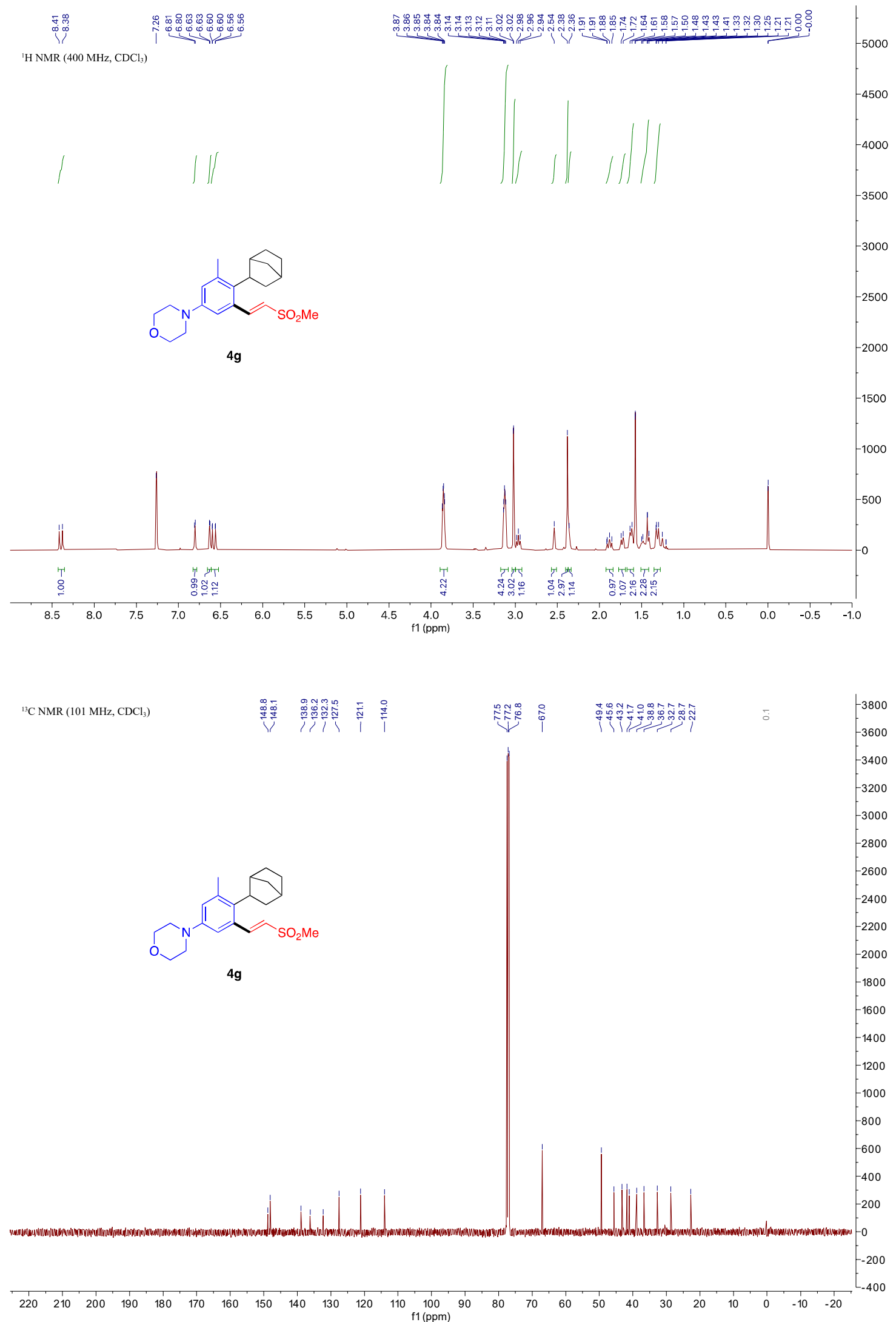

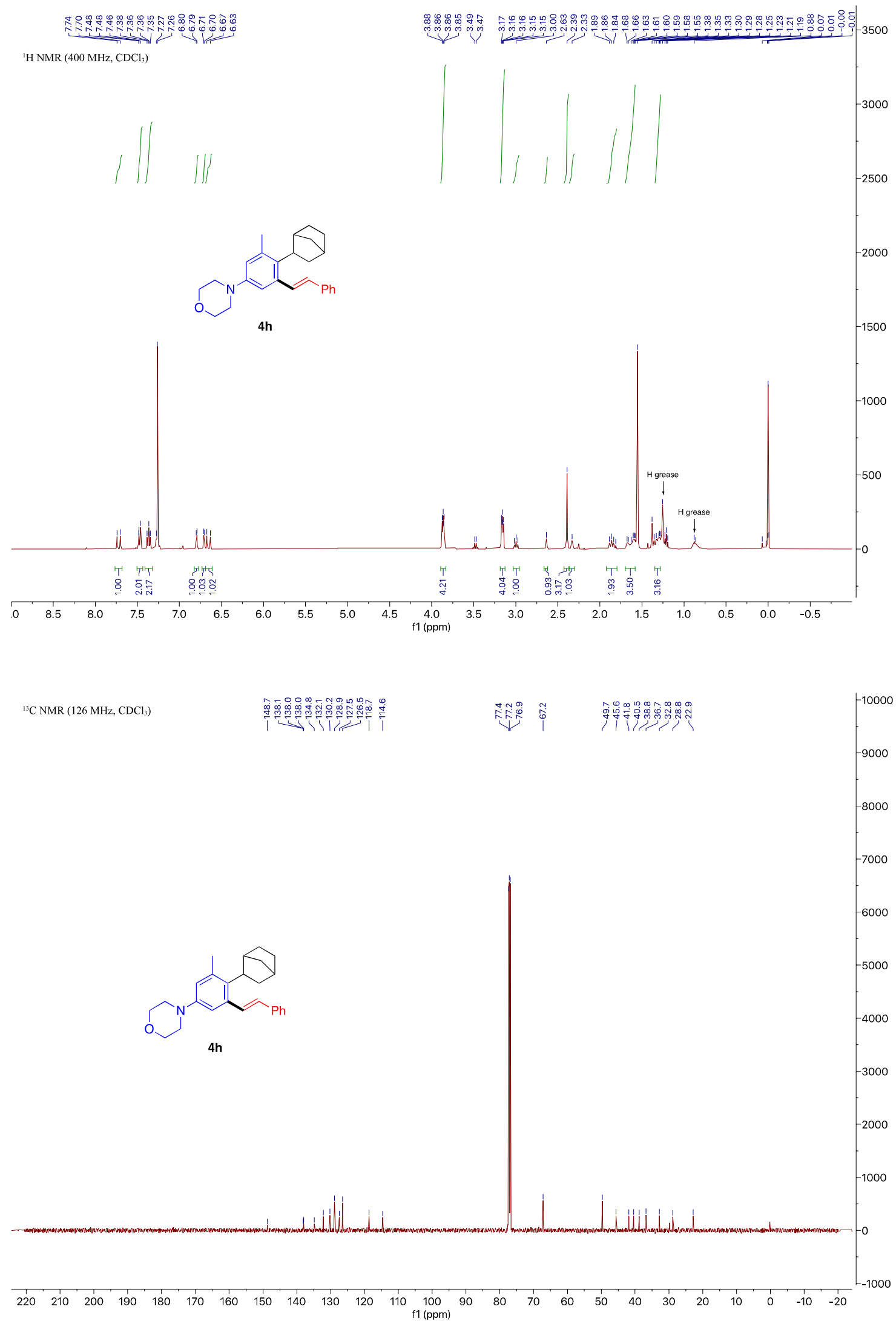

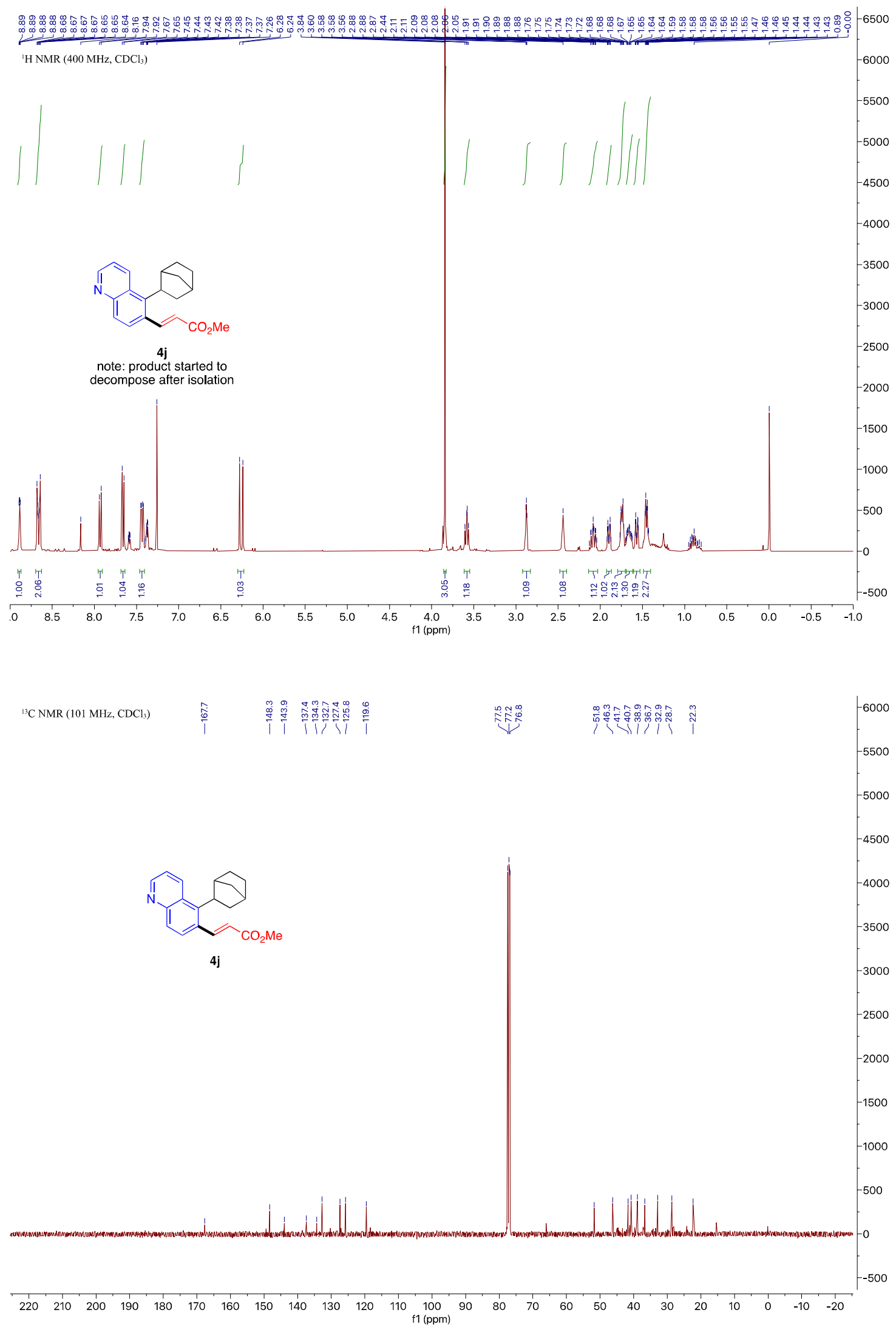

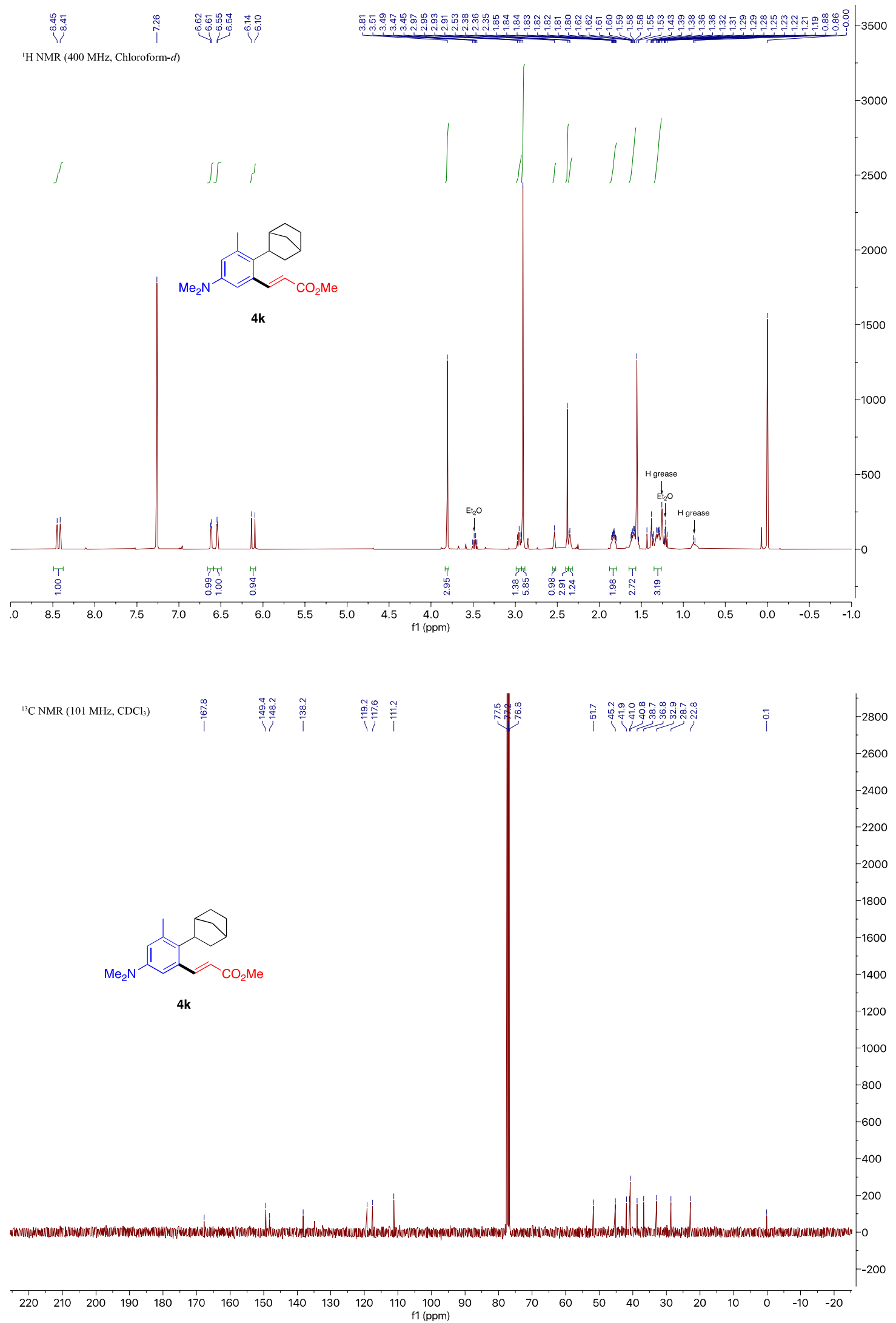


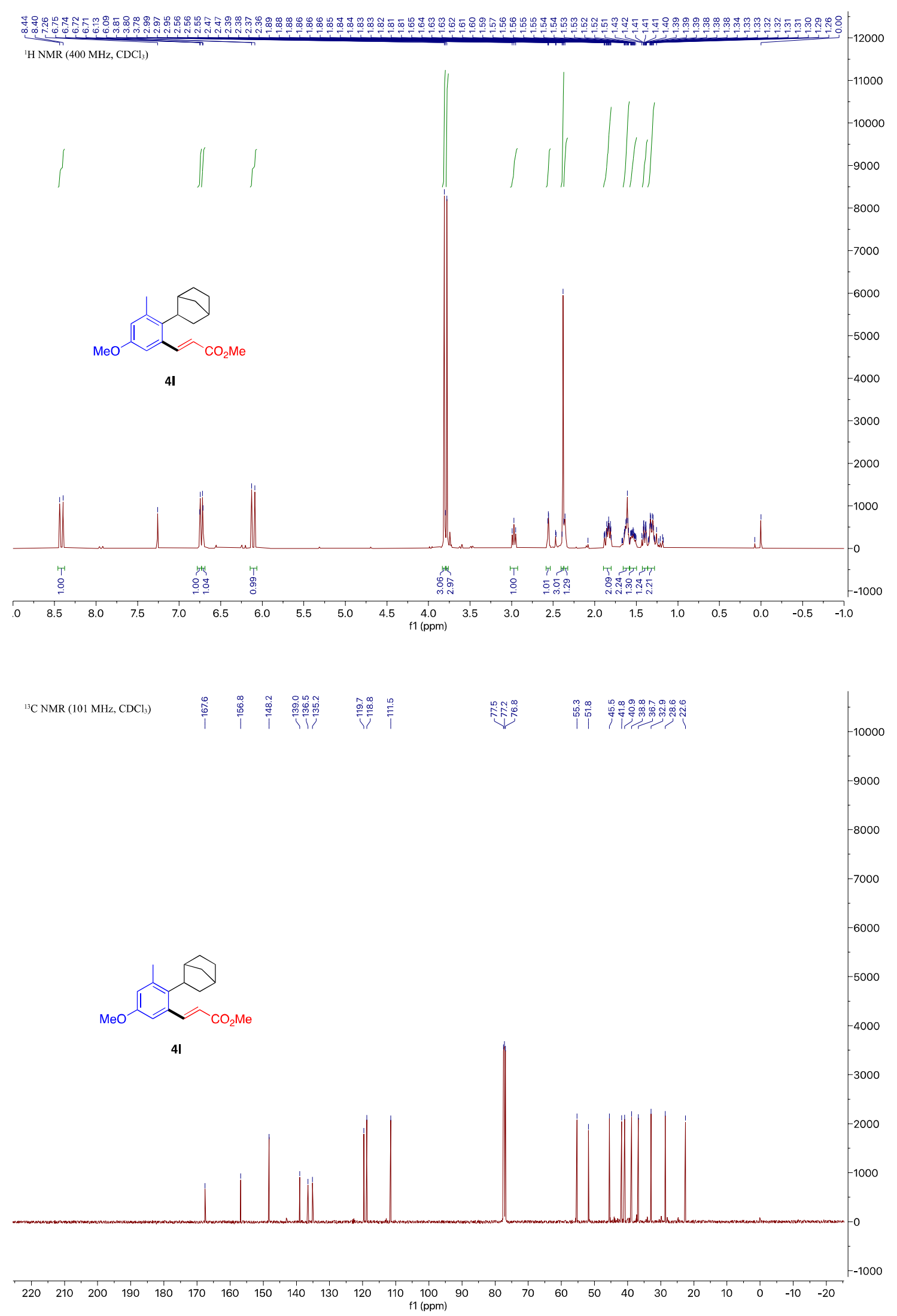



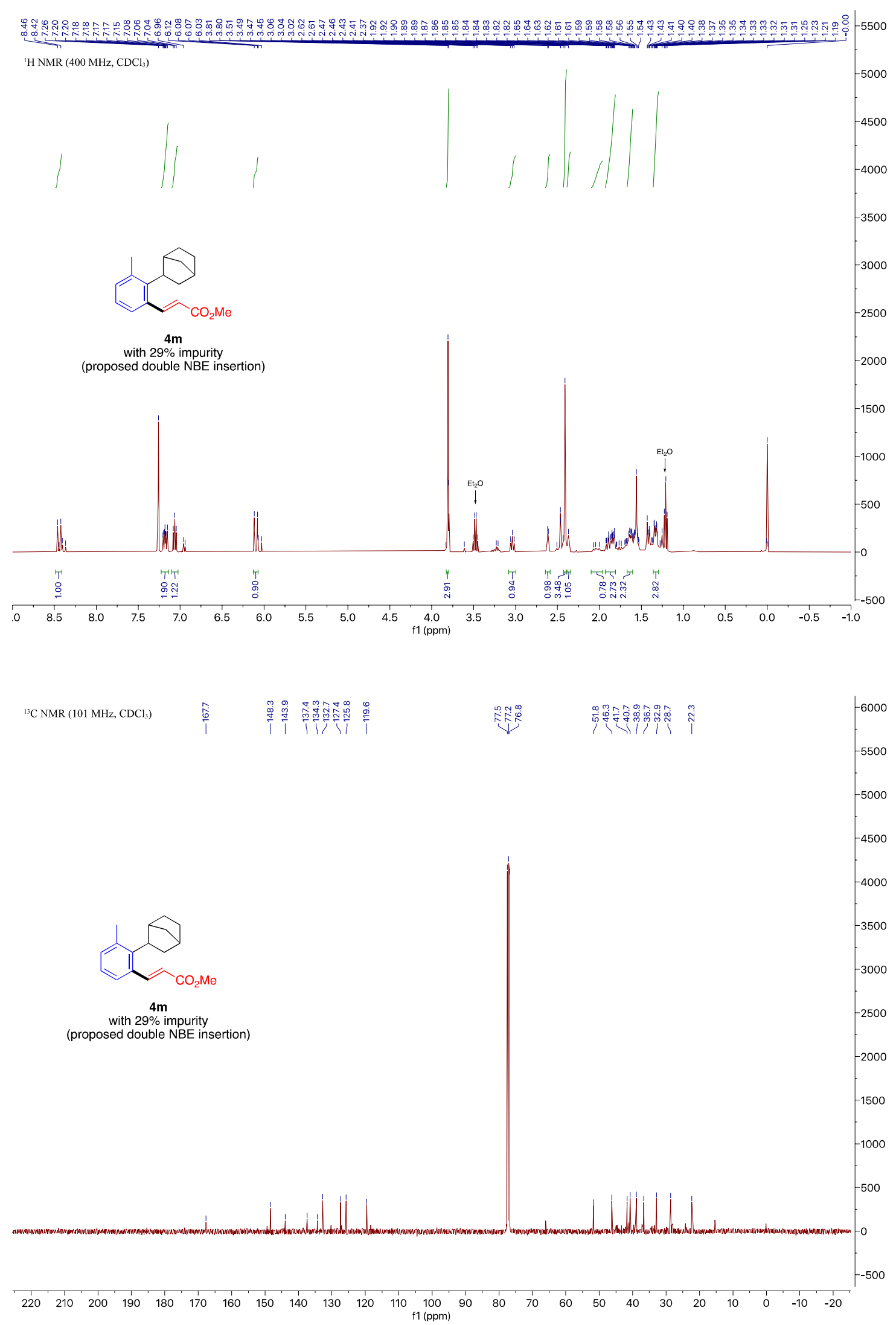

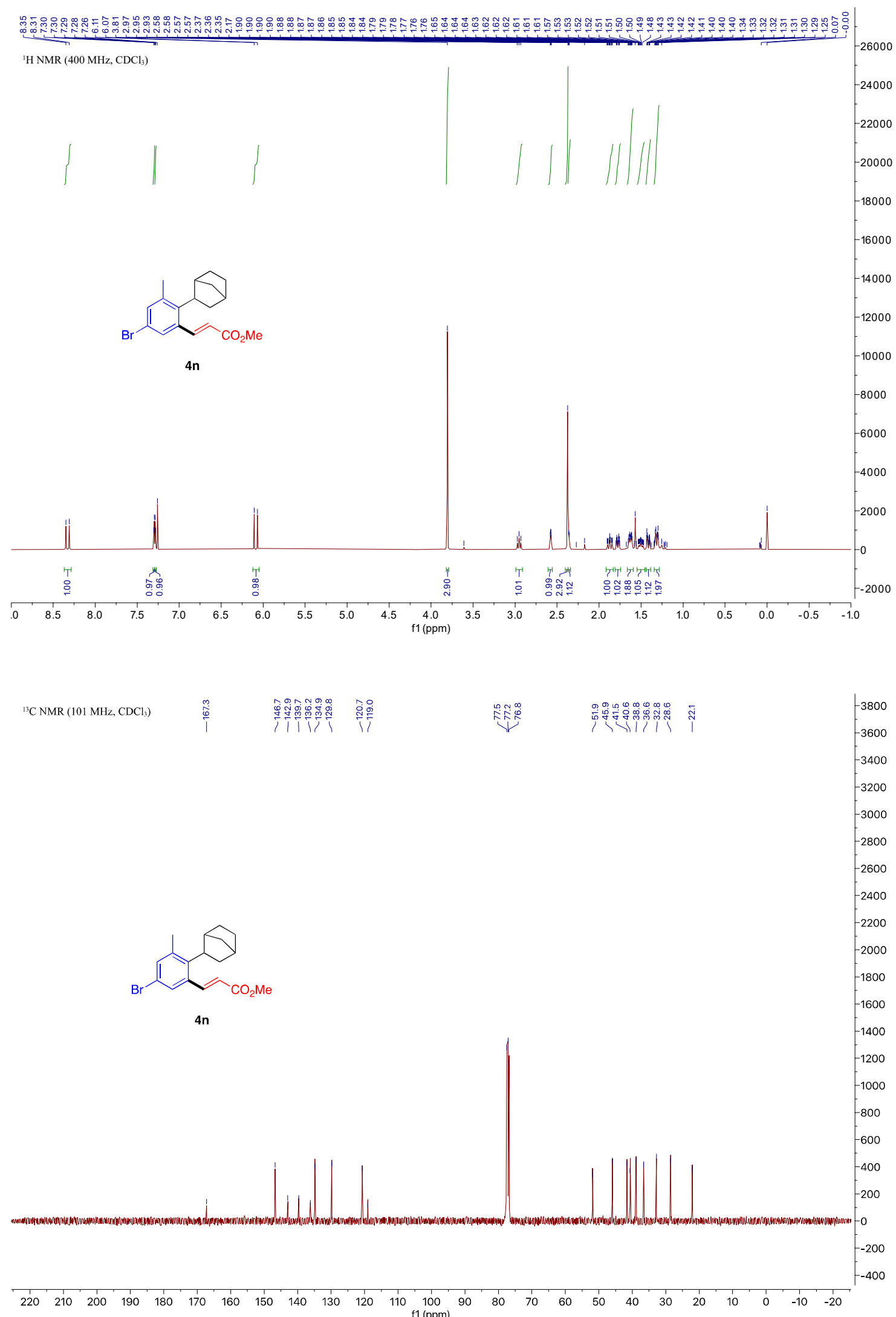


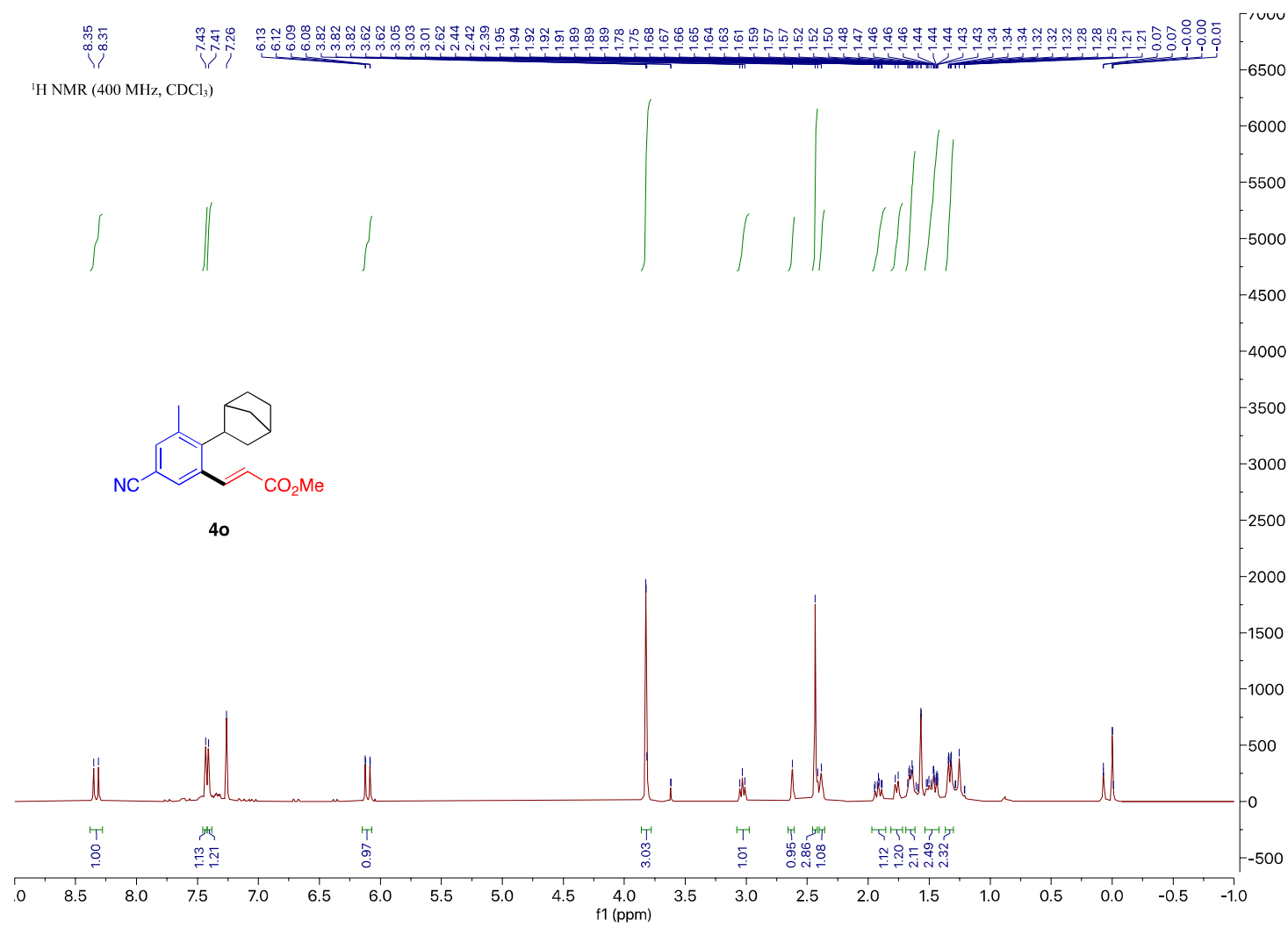

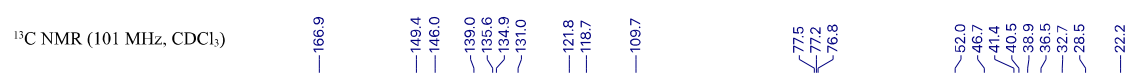

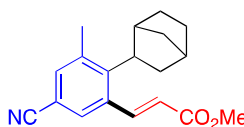

40

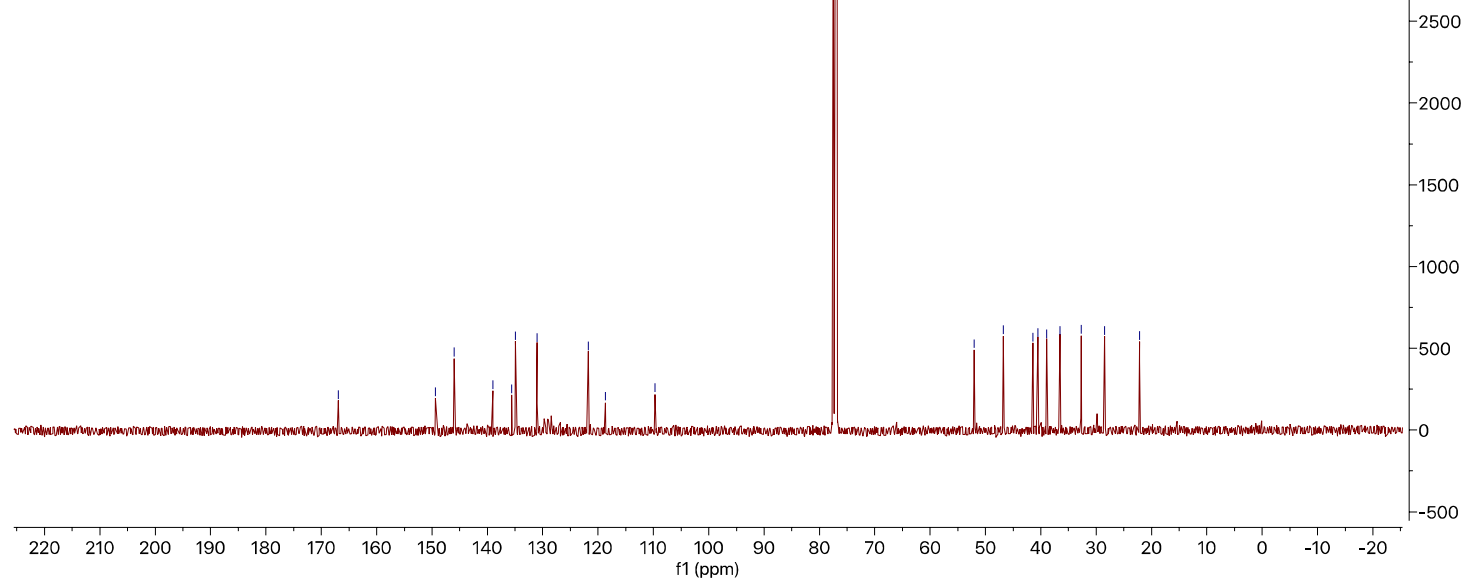




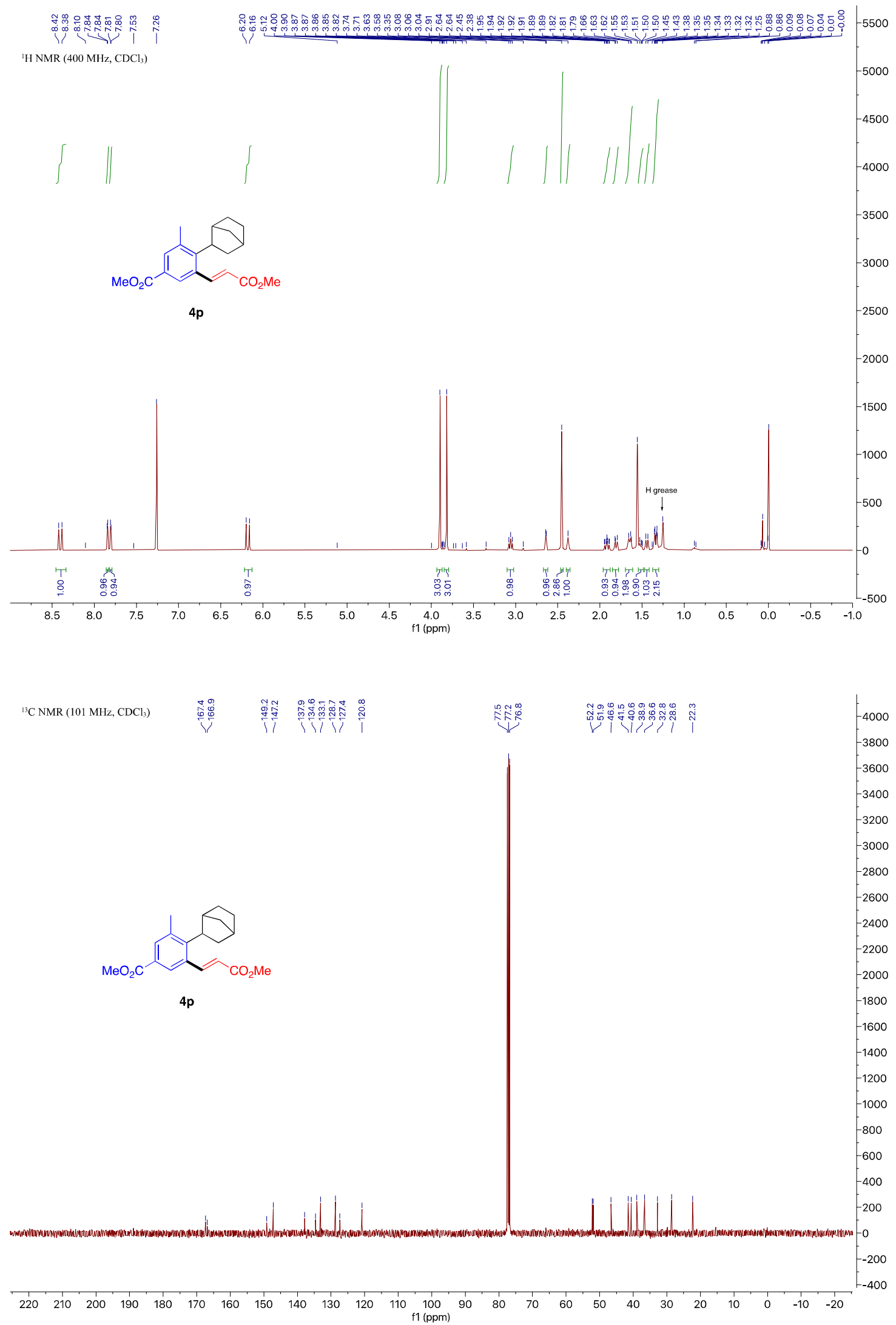



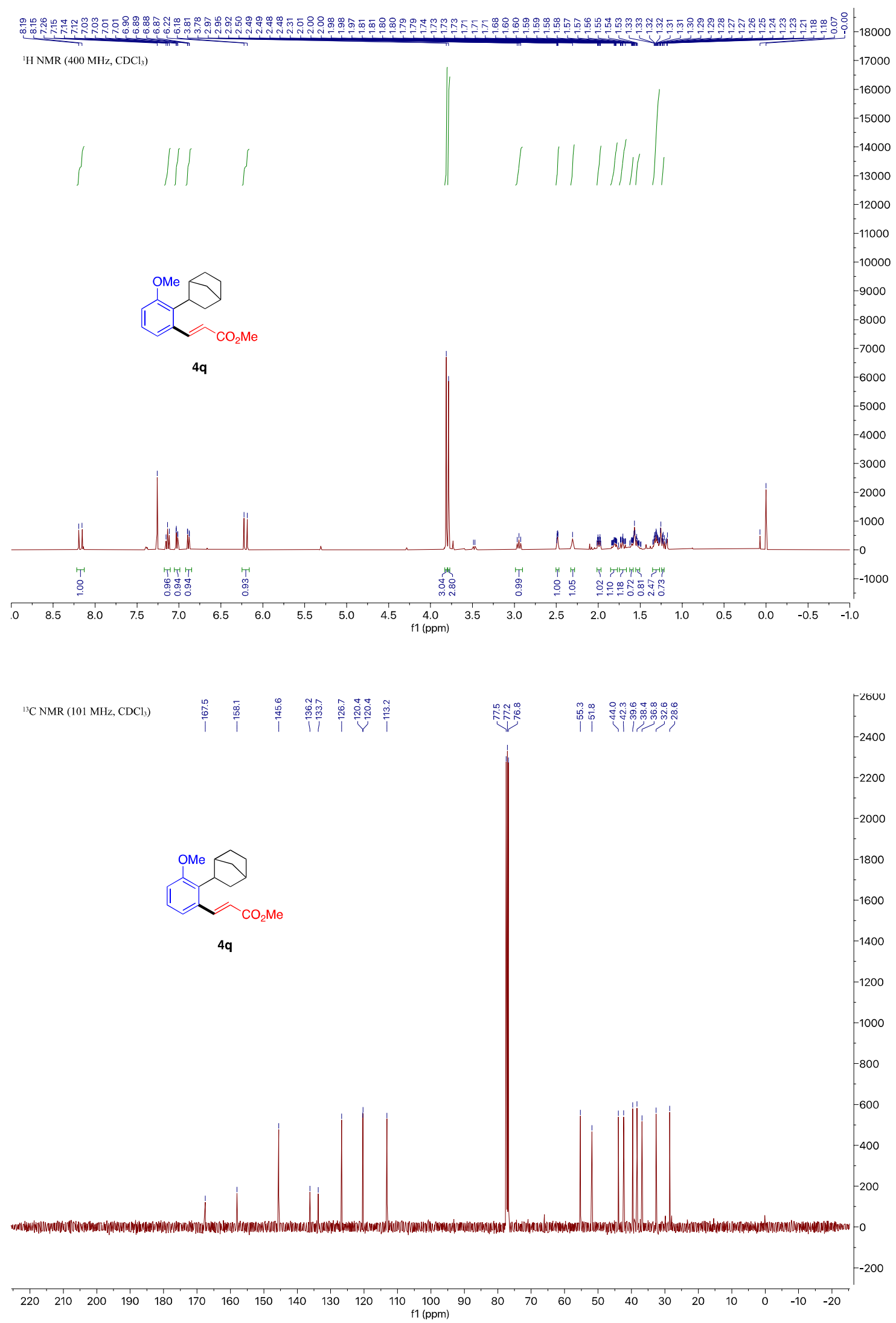


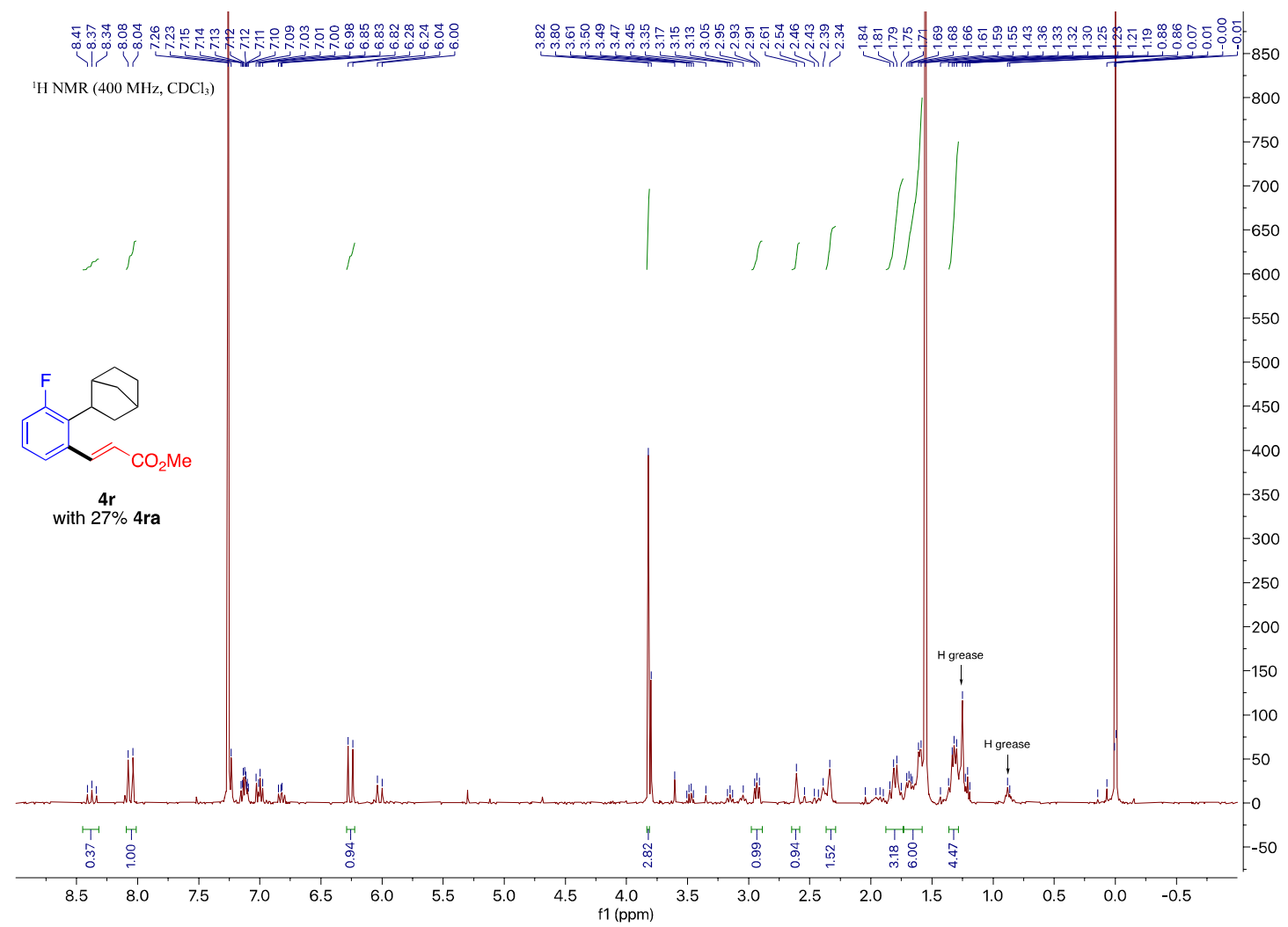

${ }^{19} \mathrm{~F} \mathrm{NMR}\left(376 \mathrm{MHz}, \mathrm{CDCl}_{3}\right)$

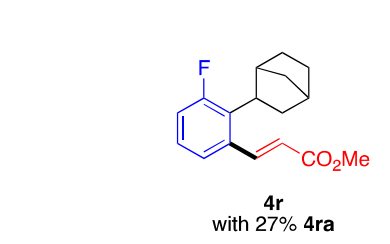

în
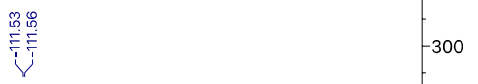


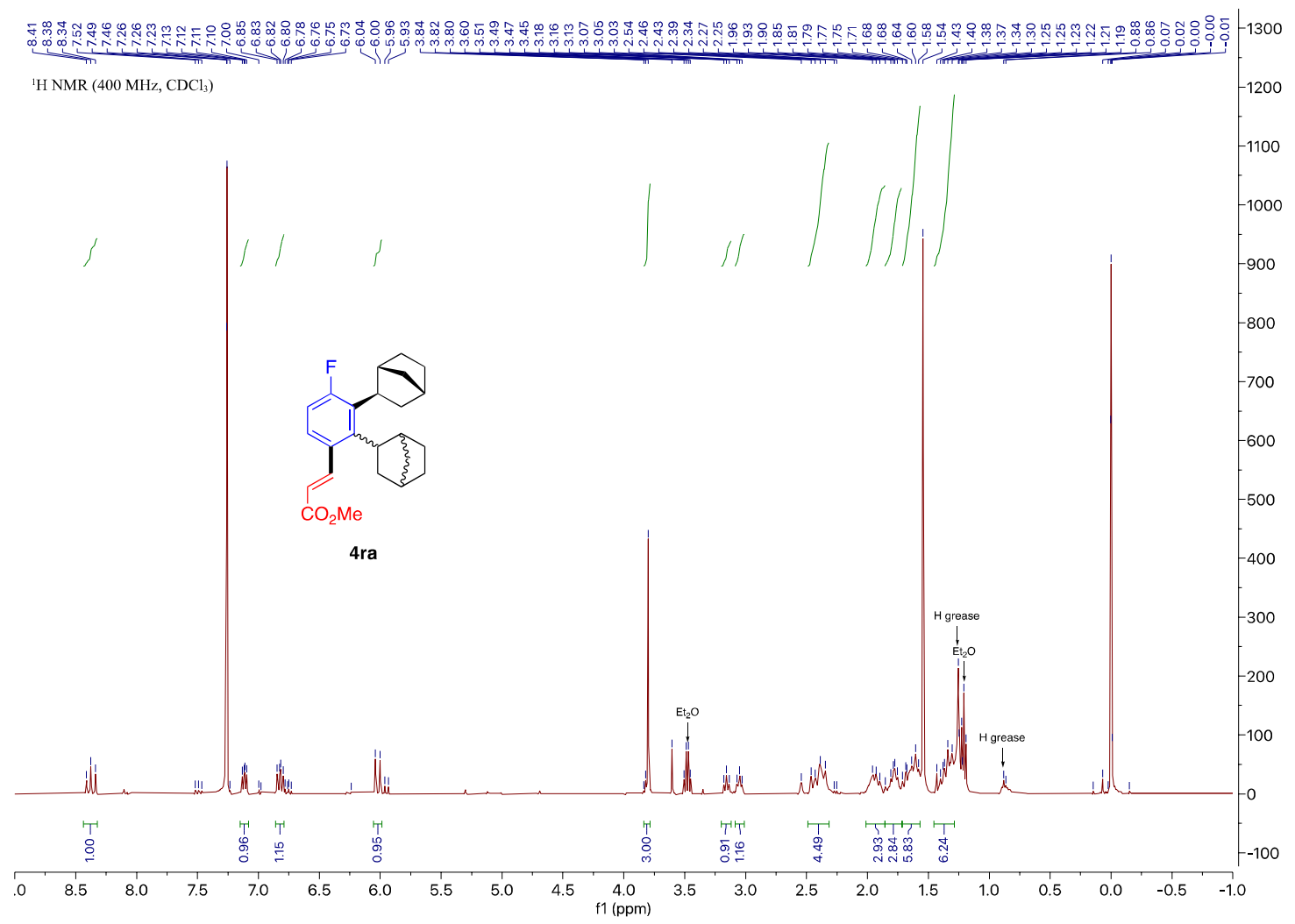

${ }^{19} \mathrm{~F}$ NMR $\left(376 \mathrm{MHz}, \mathrm{CDCl}_{3}\right)$

象
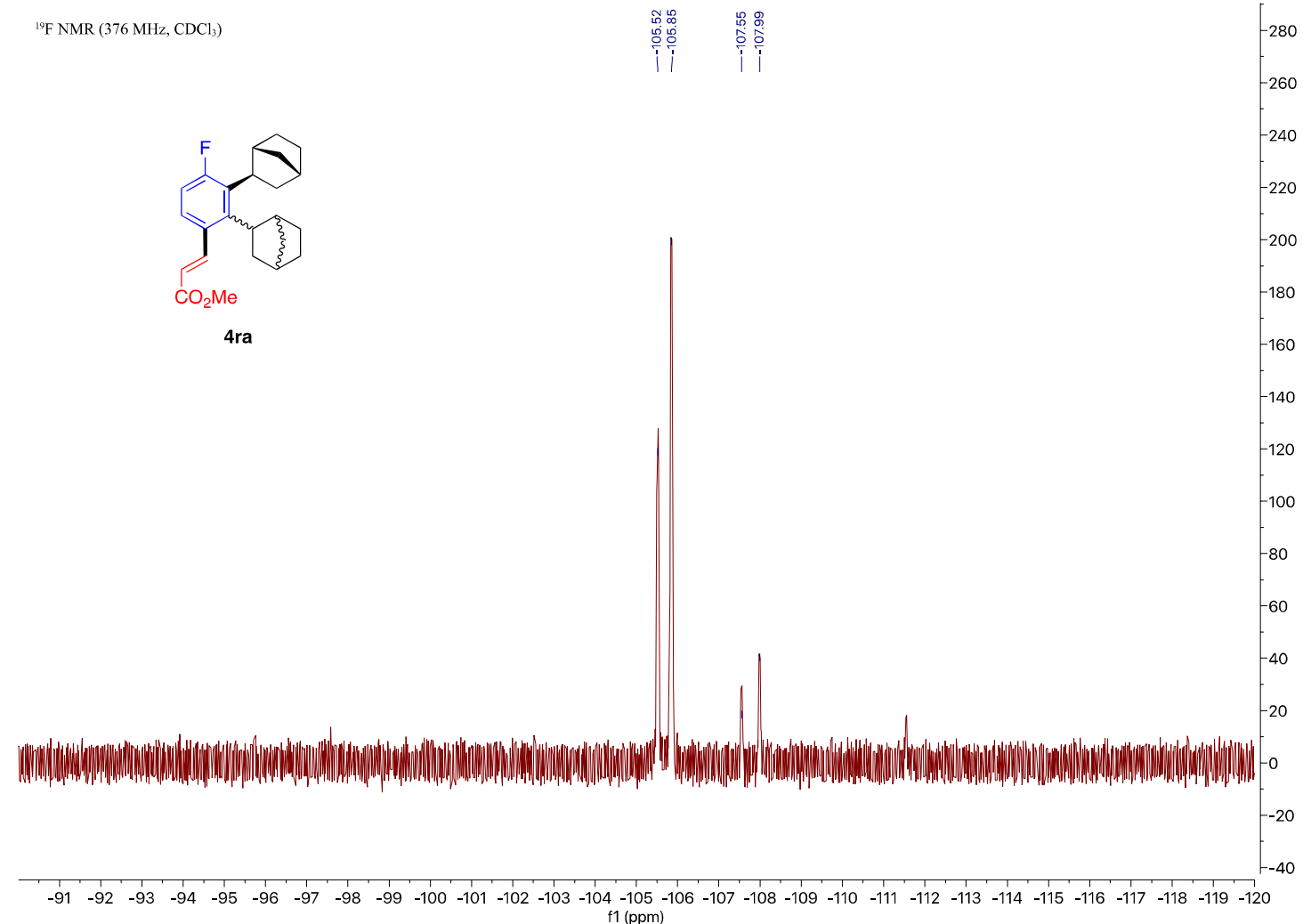


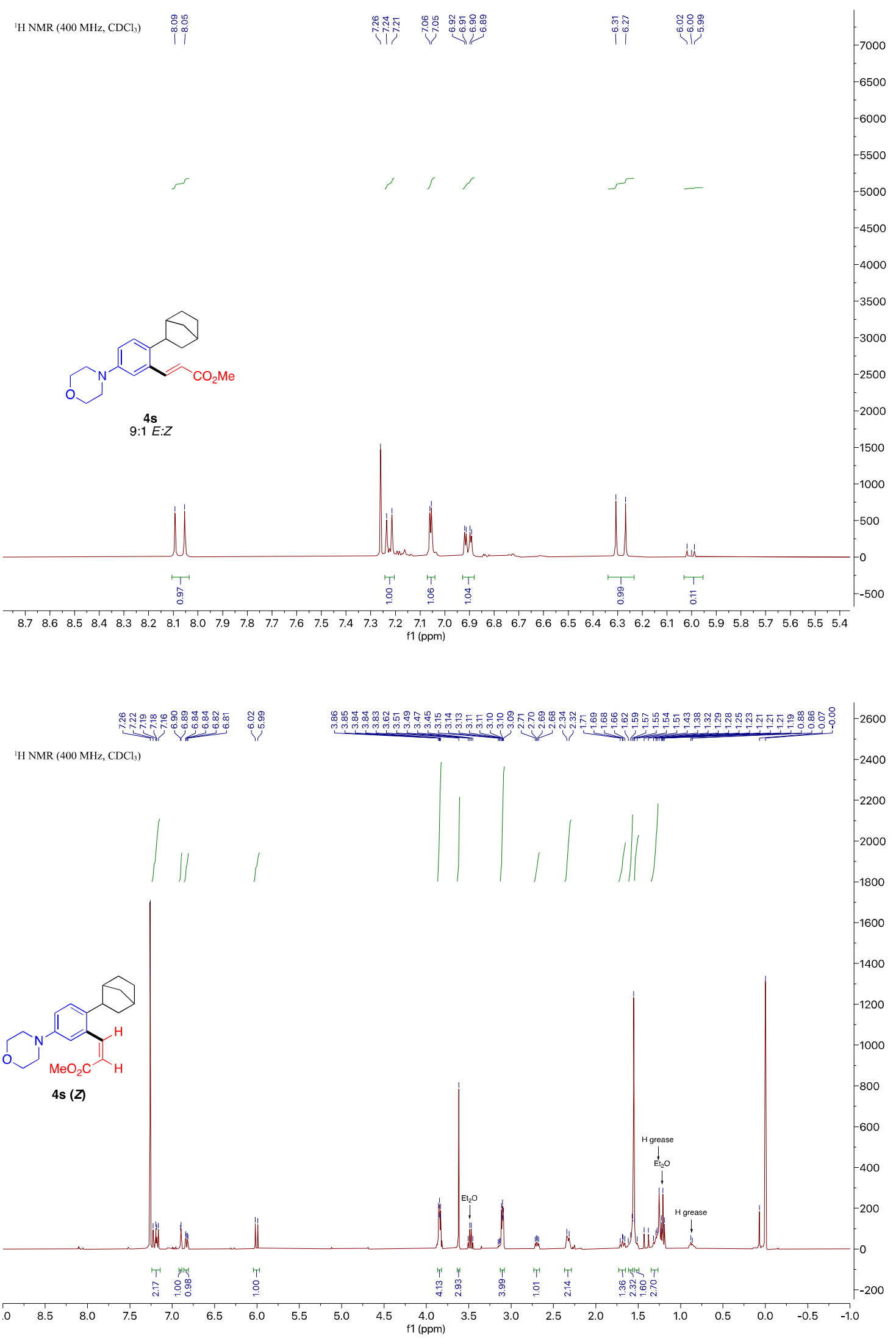




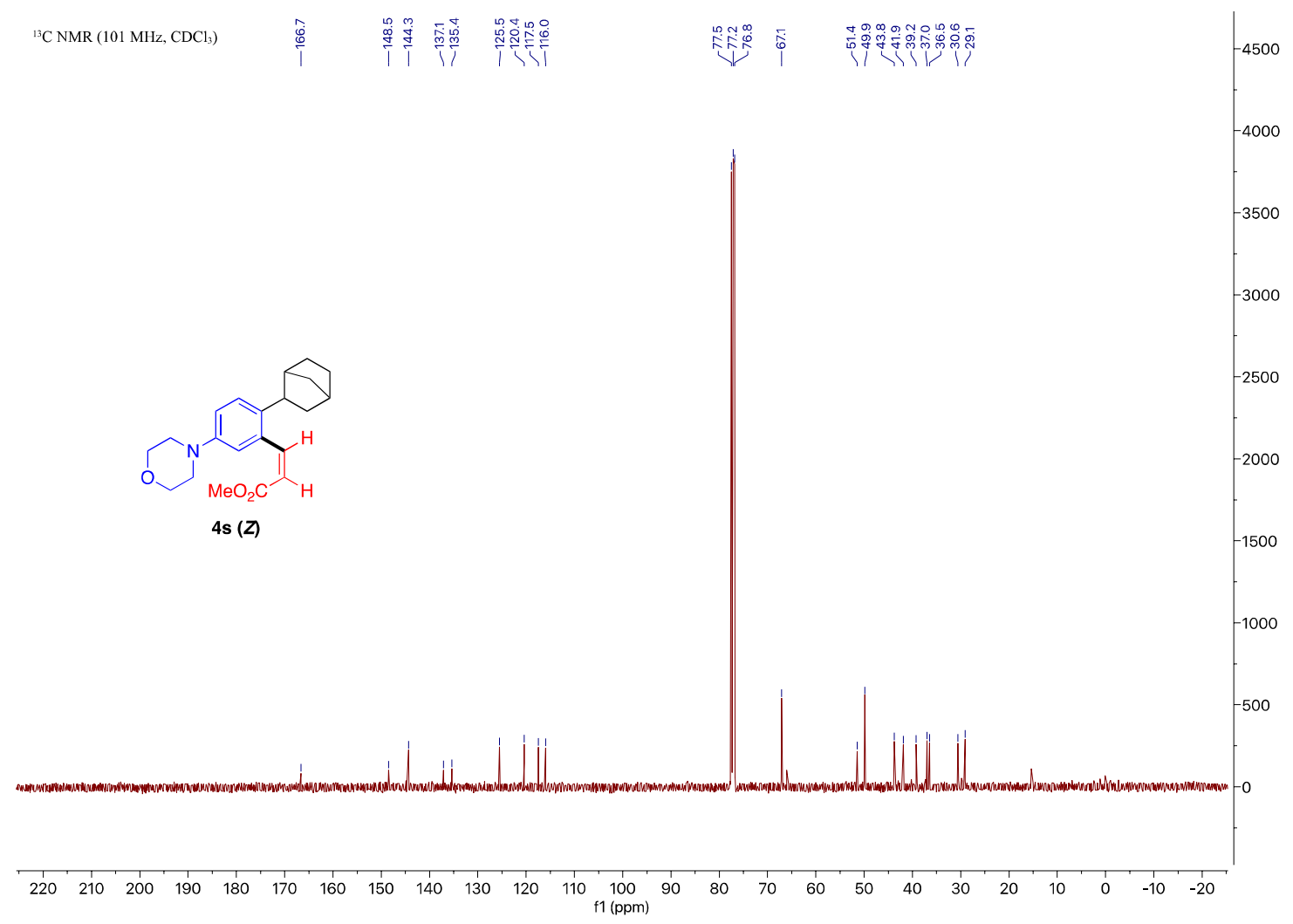



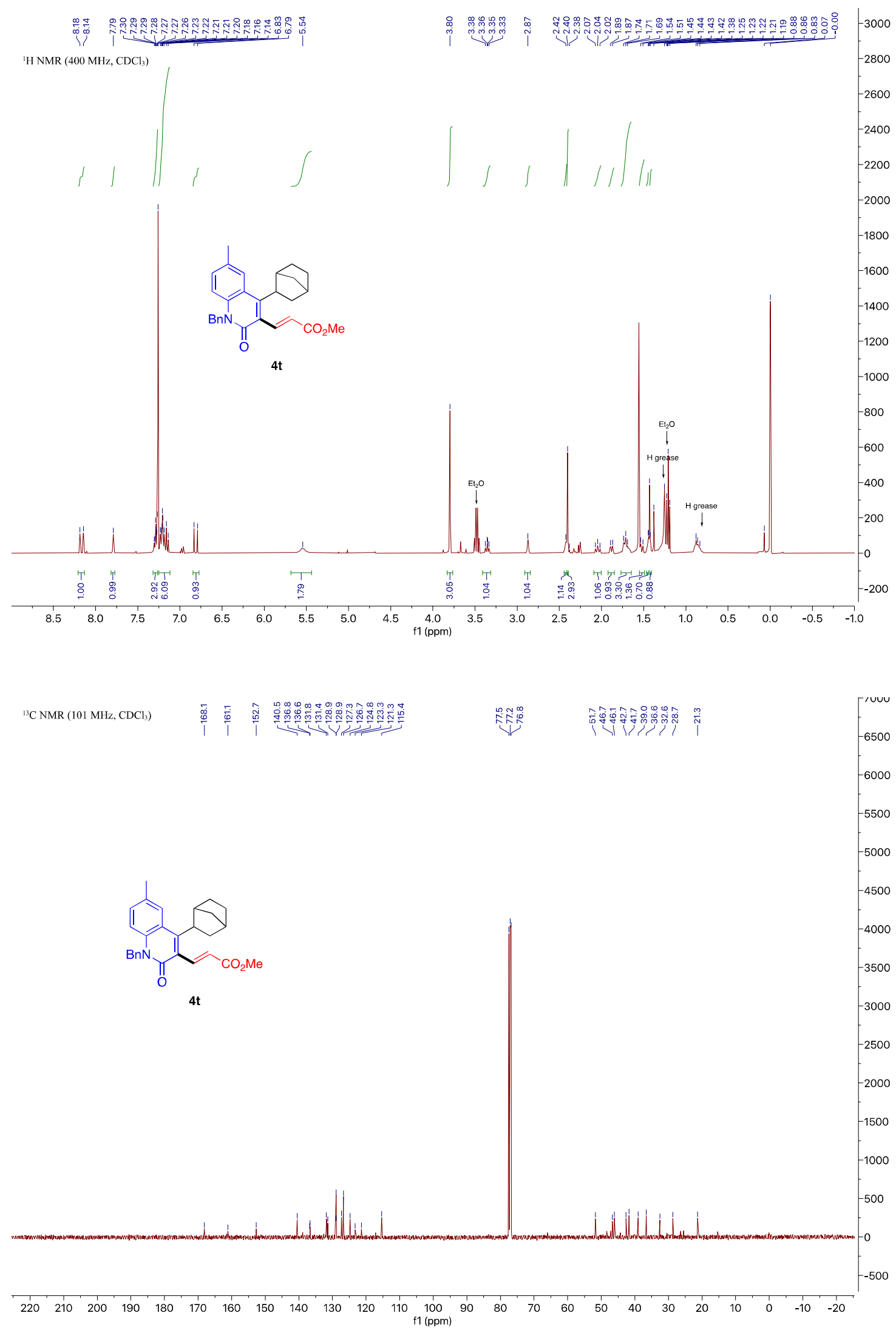

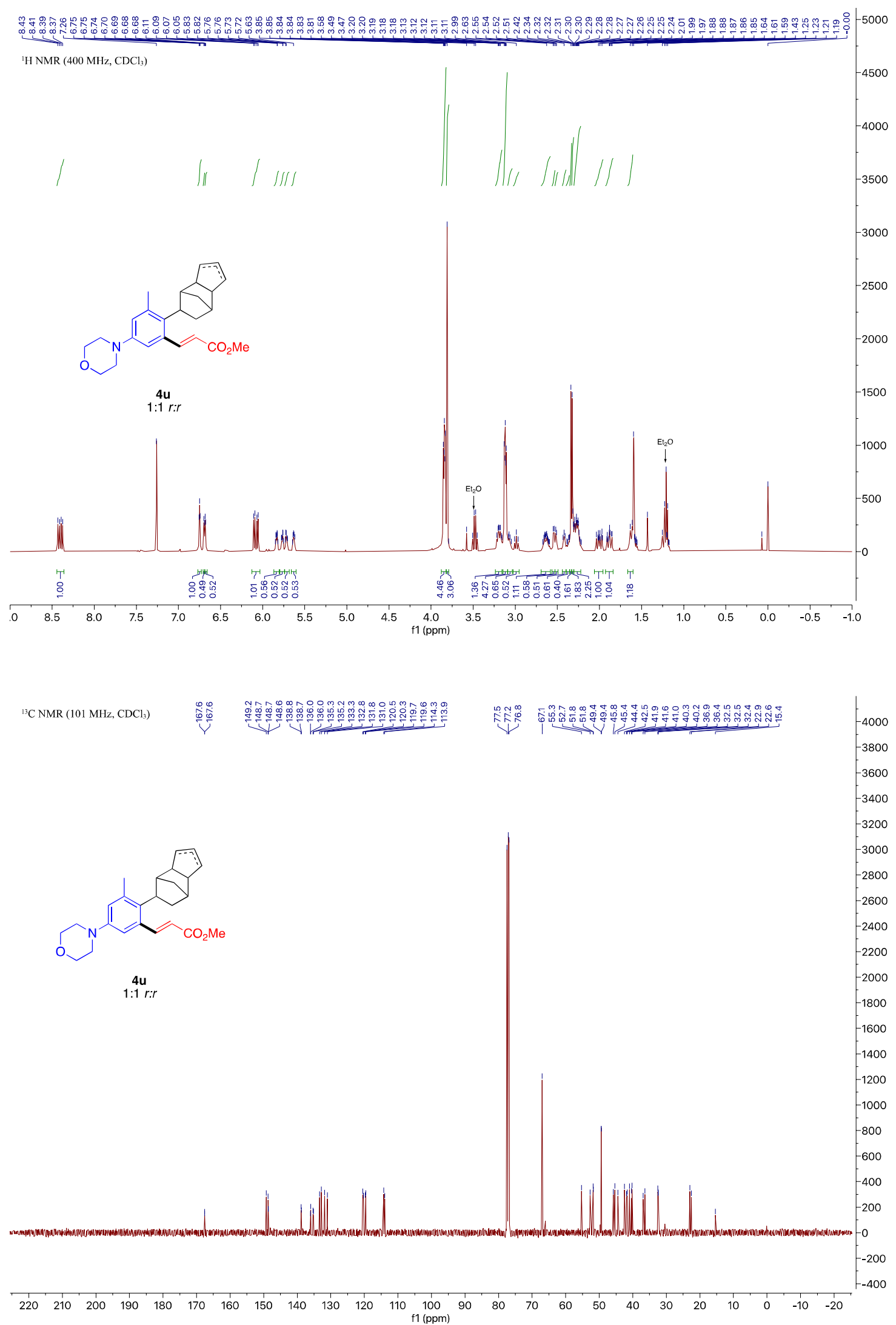


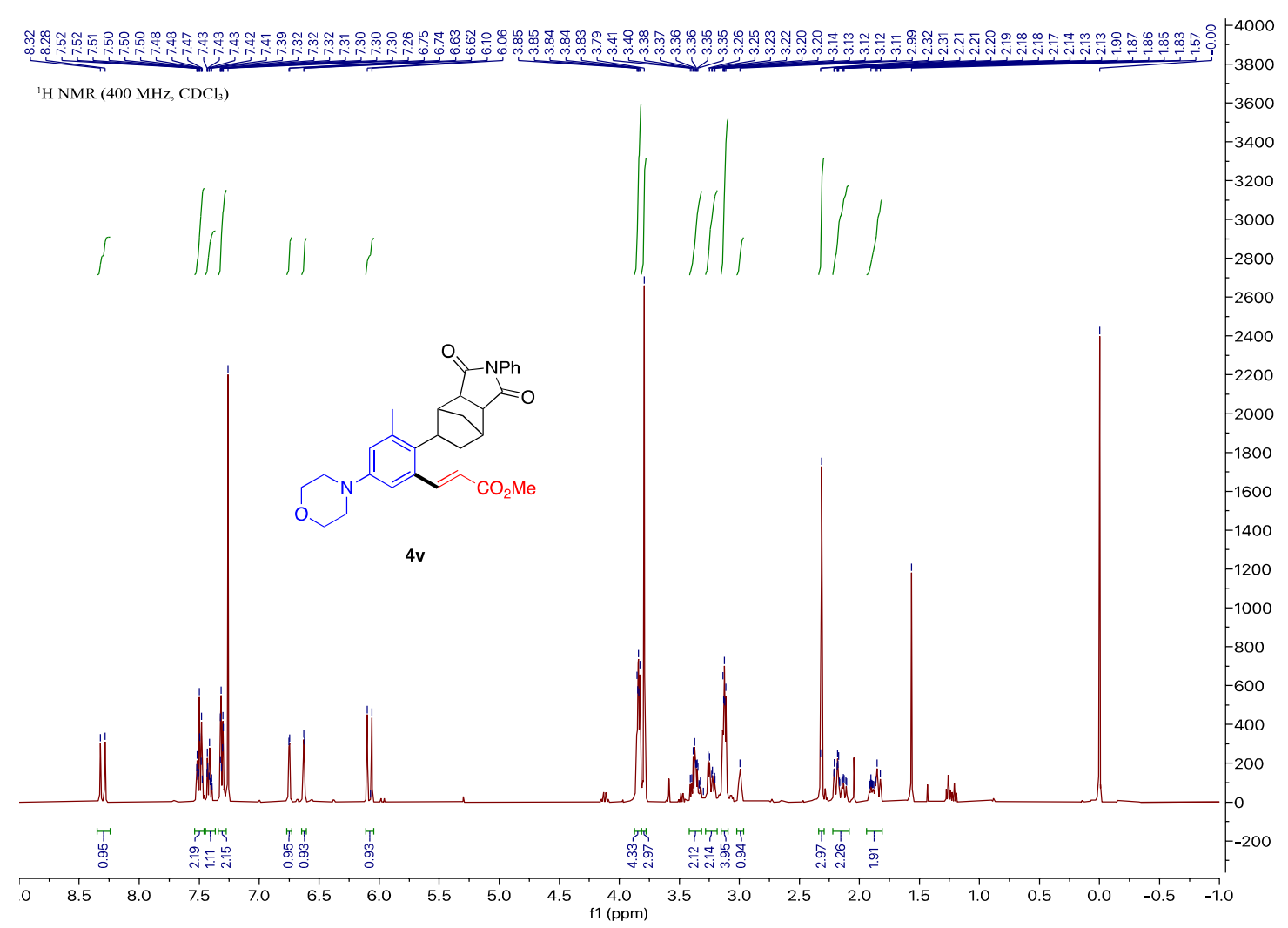

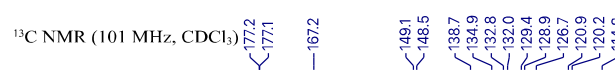

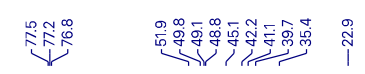

-2200
-2100

2000

$-1900$

1800

$-1700$

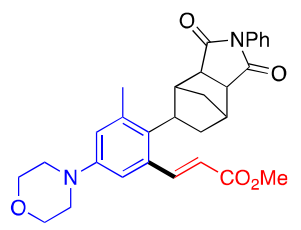

$4 v$

$-1500$

1400

$-1300$

1200

$-1100$

$-1000$

900

800

700

600

500

$-400$

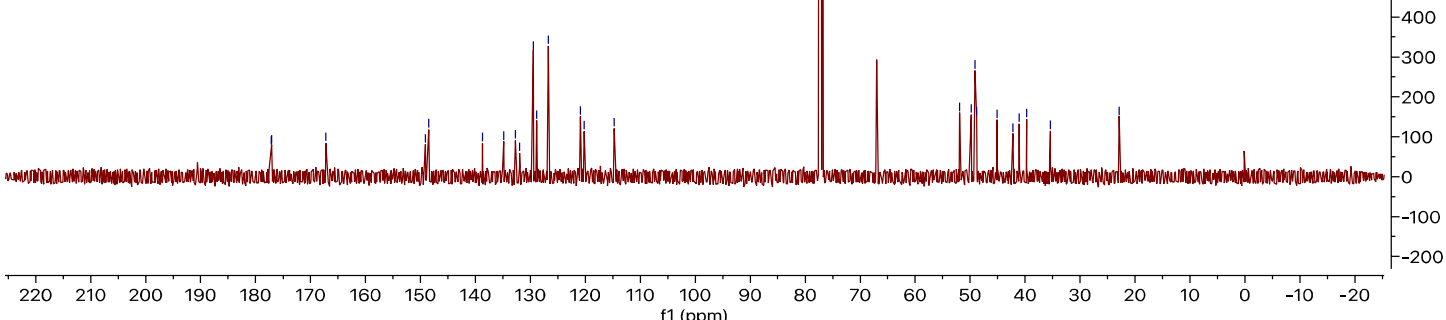

